\title{
Oxidant-free and catalyst-free synthesis of sulfonated benzothiophenes via electrooxidative tandem cyclization
}

Dong Zhang, ${ }^{\mathrm{a} \dagger}$ Jinlin Cai, ${ }^{\mathrm{a}} \dagger$ Jinze Du, ${ }^{\mathrm{a}}$ Xujia Wang, ${ }^{\mathrm{a}}$ Wei He, ${ }^{\mathrm{a}}$ Zhao Yang, ${ }^{\mathrm{b}}$ Chengkou Liu,,${ }^{\mathrm{a}, *}$ Zheng Fang, ${ }^{\text {a, }}{ }^{*}$ and Kai Guo a,c

${ }^{a}$ College of Biotechnology and Pharmaceutical Engineering Nanjing Tech University, 30 Puzhu Rd S., Nanjing, 211816, China Email: guok@njtech.edu.cn, Tel +86 255813 9901, Fax +86 255813 9935.

${ }^{\mathrm{b}}$ College of Engineering China Pharmaceutical University, 24 Tongjiaxiang, Nanjing, 210003, China

c State Key Laboratory of Materials-Oriented Chemical Engineering, 30 Puzhu Rd S., Nanjing, 211816, China

† These authors contributed to the work equally.

Table of Contents

1. ${ }^{1} \mathrm{H}$ NMR, ${ }^{19}$ F NMR and ${ }^{13} \mathrm{C}\left\{{ }^{1} \mathrm{H}\right\}$ NMR spectra 


\section{1. ${ }^{1} \mathrm{H}$ NMR, ${ }^{19}$ F NMR and ${ }^{13} \mathrm{C}$ NMR spectra}

2-Phenyl-3-tosylbenzo[b]thiophene (3a)

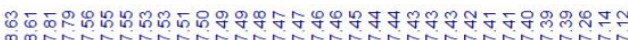
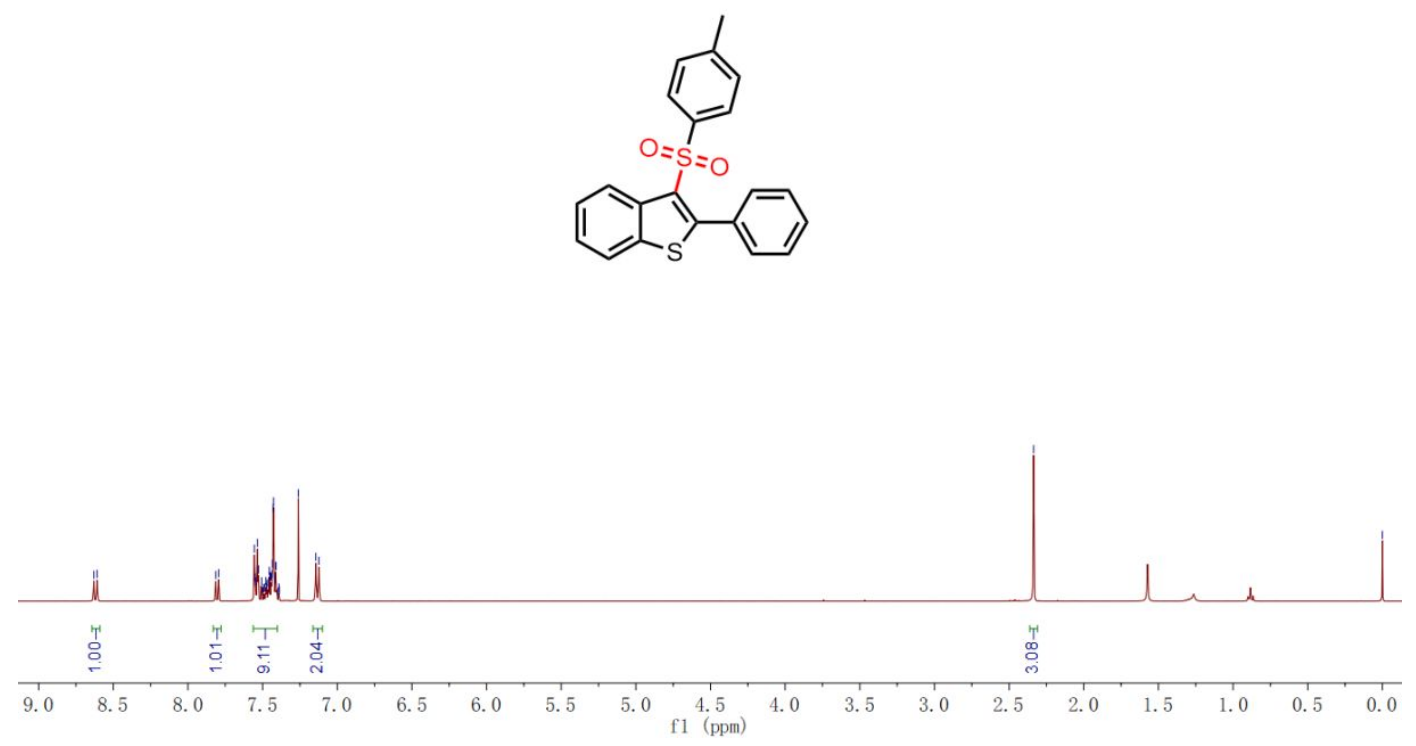

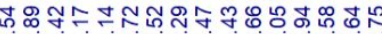
กิ่

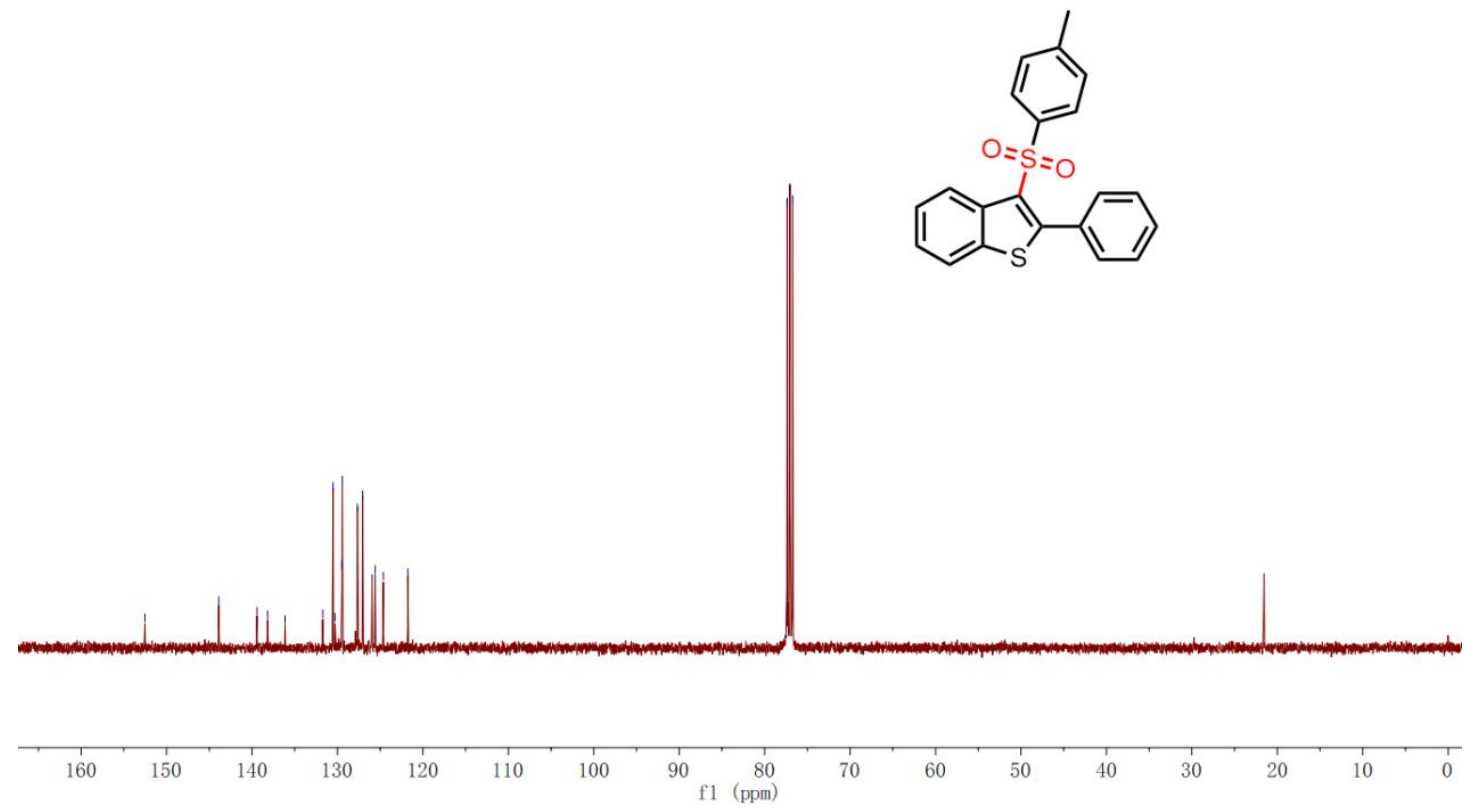

Figure S1. ${ }^{1} \mathrm{H}$ NMR of 3a $\left(400 \mathrm{MHz}, \mathrm{CDCl}_{3}\right)$ and ${ }^{13} \mathrm{C}\left\{{ }^{1} \mathrm{H}\right\}$ NMR of 3a $\left(100 \mathrm{MHz}, \mathrm{CDCl}_{3}\right)$ 
2-(o-Tolyl)-3-tosylbenzo[b]thiophene (3b)

ติ

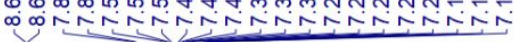

$\stackrel{\infty}{\text { i }} \underset{i}{\stackrel{0}{i}}$

\&
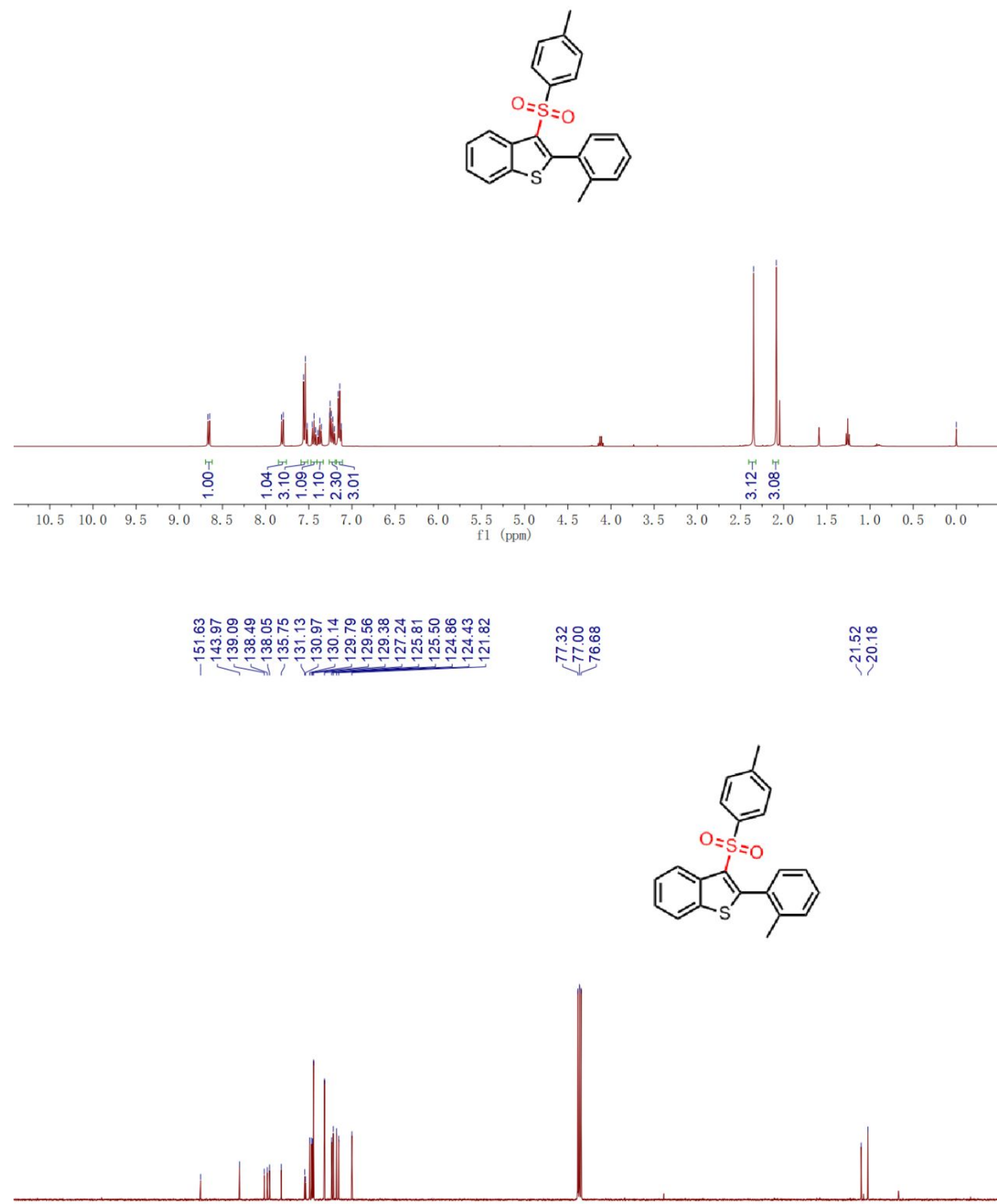

$\begin{array}{llllllllll}180 & 170 & 160 & 150 & 140 & 130 & 120 & 110 & 100 & 90\end{array}$ $\mathrm{f1}(\mathrm{ppm})$

Figure S2. ${ }^{1} \mathrm{H}$ NMR of $\mathbf{3 b}\left(400 \mathrm{MHz}, \mathrm{CDCl}_{3}\right)$ and ${ }^{13} \mathrm{C}\left\{{ }^{1} \mathrm{H}\right\}$ NMR of $\mathbf{3 b}\left(100 \mathrm{MHz}, \mathrm{CDCl}_{3}\right)$ 
2-(2-Fluorophenyl)-3-tosylbenzo[b]thiophene (3c)

舟

$\stackrel{\text { }}{\text { i }}$

웅

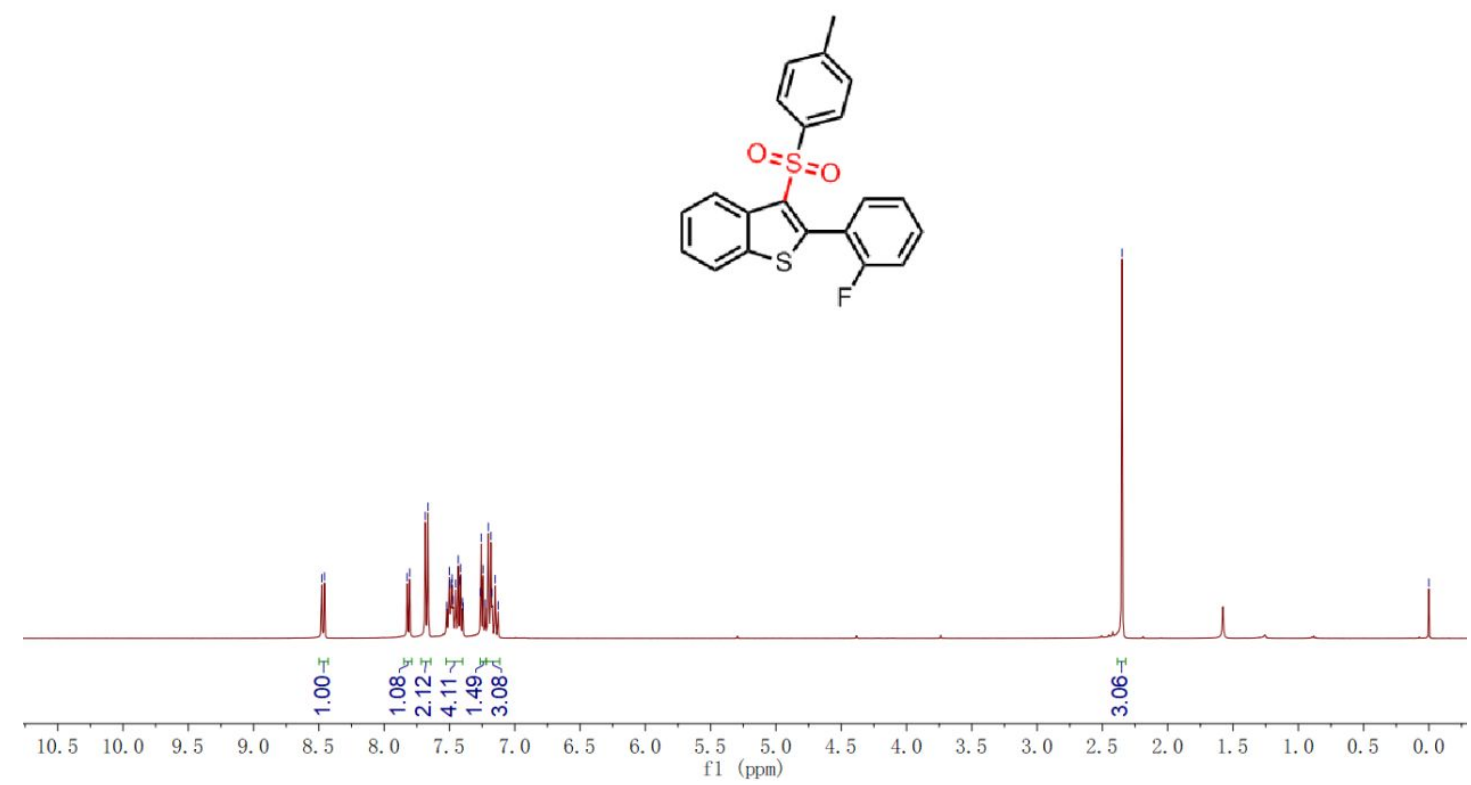

웜
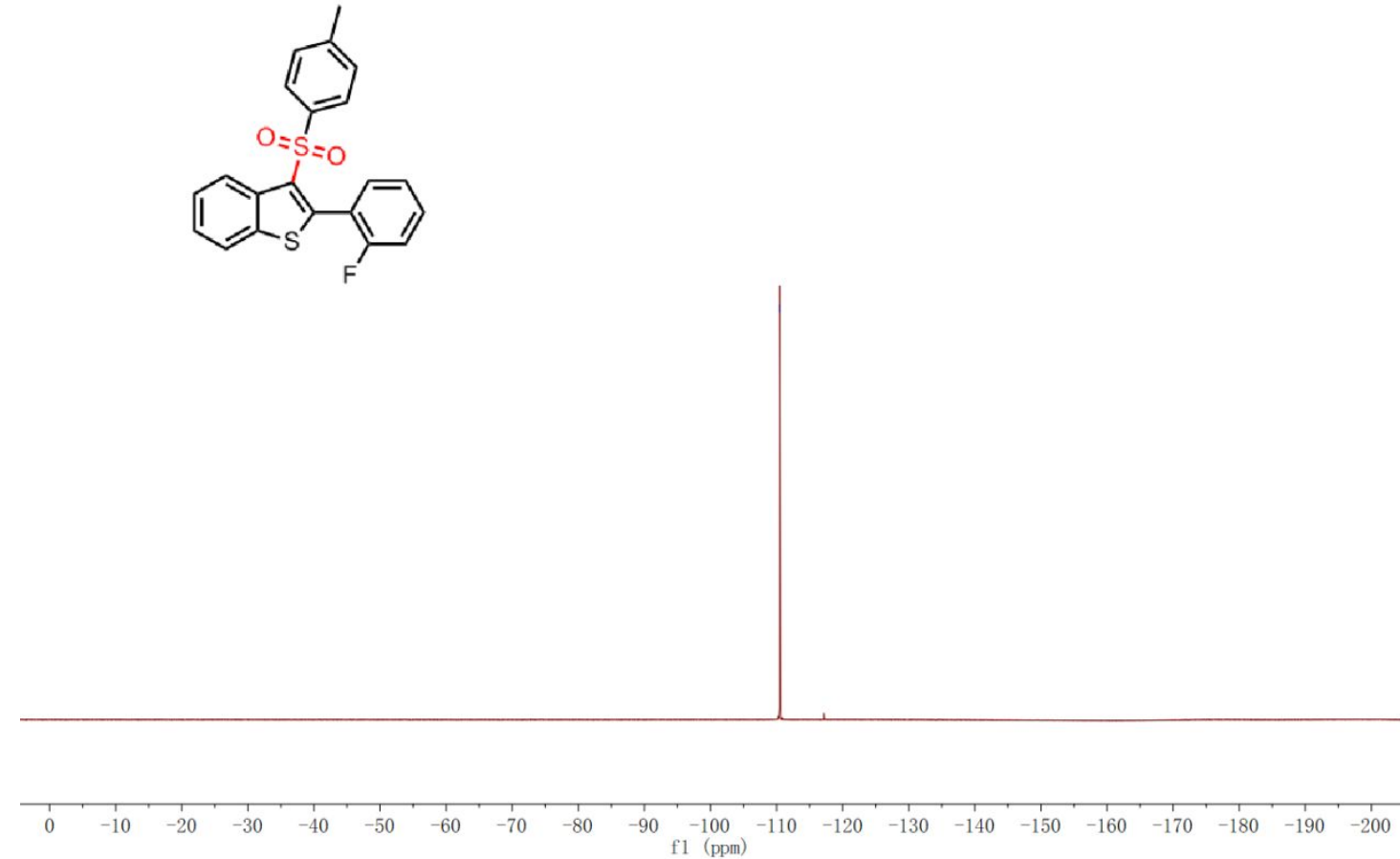


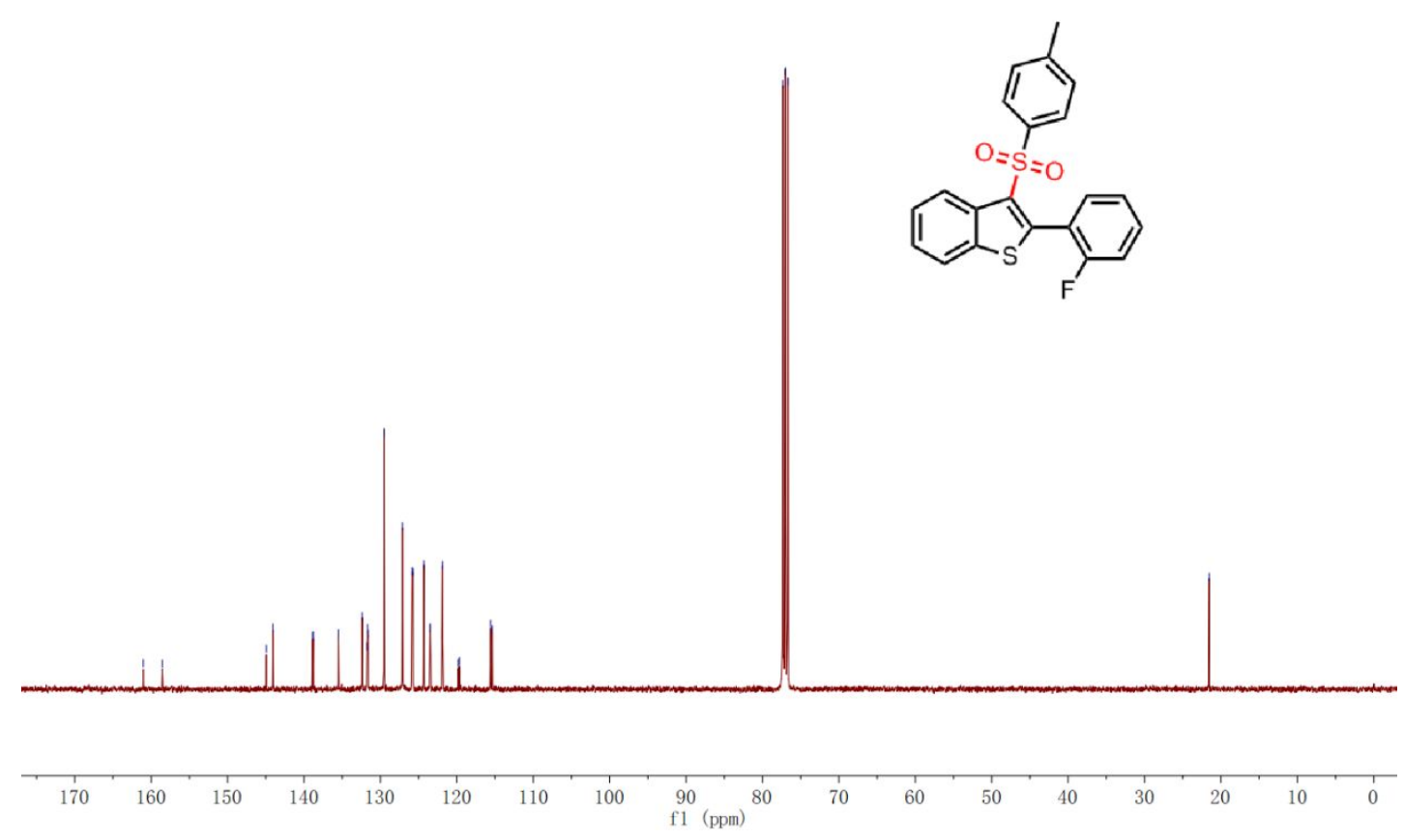

Figure S3. ${ }^{1} \mathrm{H}$ NMR of $\mathbf{3 c}\left(400 \mathrm{MHz}, \mathrm{CDCl}_{3}\right),{ }^{19} \mathrm{~F}$ NMR of $\mathbf{3 c}\left(376 \mathrm{MHz}, \mathrm{CDCl}_{3}\right)$ and ${ }^{13} \mathrm{C}\left\{{ }^{1} \mathrm{H}\right\}$ NMR of $\mathbf{3 b}\left(100 \mathrm{MHz}, \mathrm{CDCl}_{3}\right)$

2-(2-Chlorophenyl)-3-tosylbenzo[b]thiophene (3d)

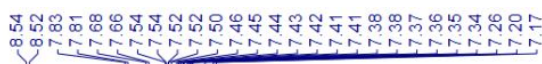<smiles>Cc1ccc(S(=O)(=O)c2c(-c3ccccc3Cl)sc3ccccc23)cc1</smiles>

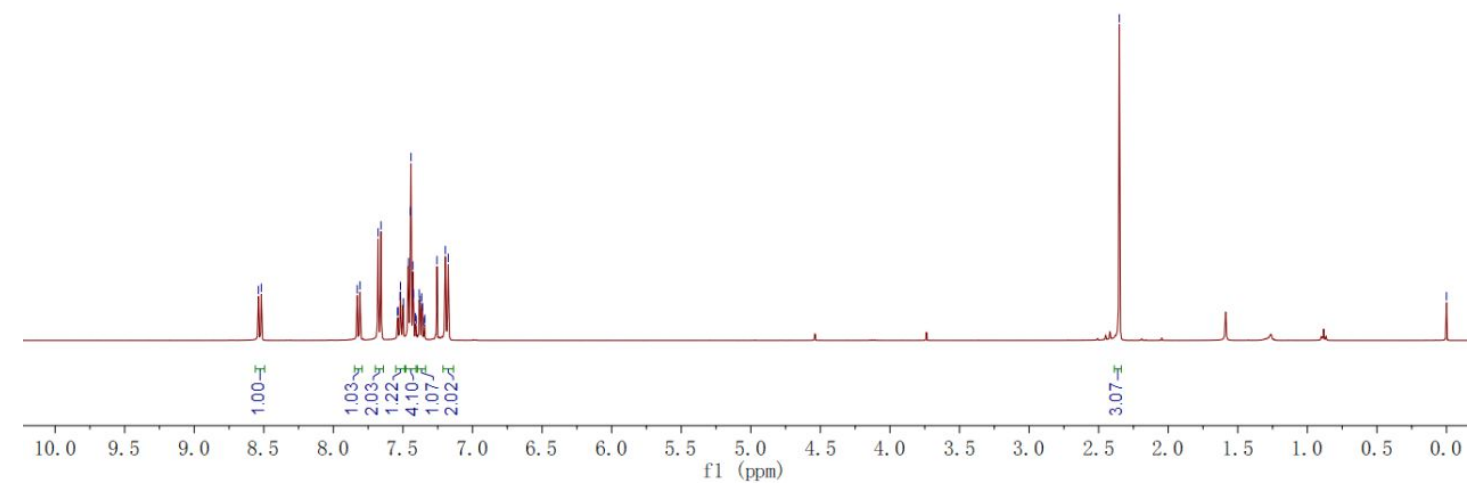



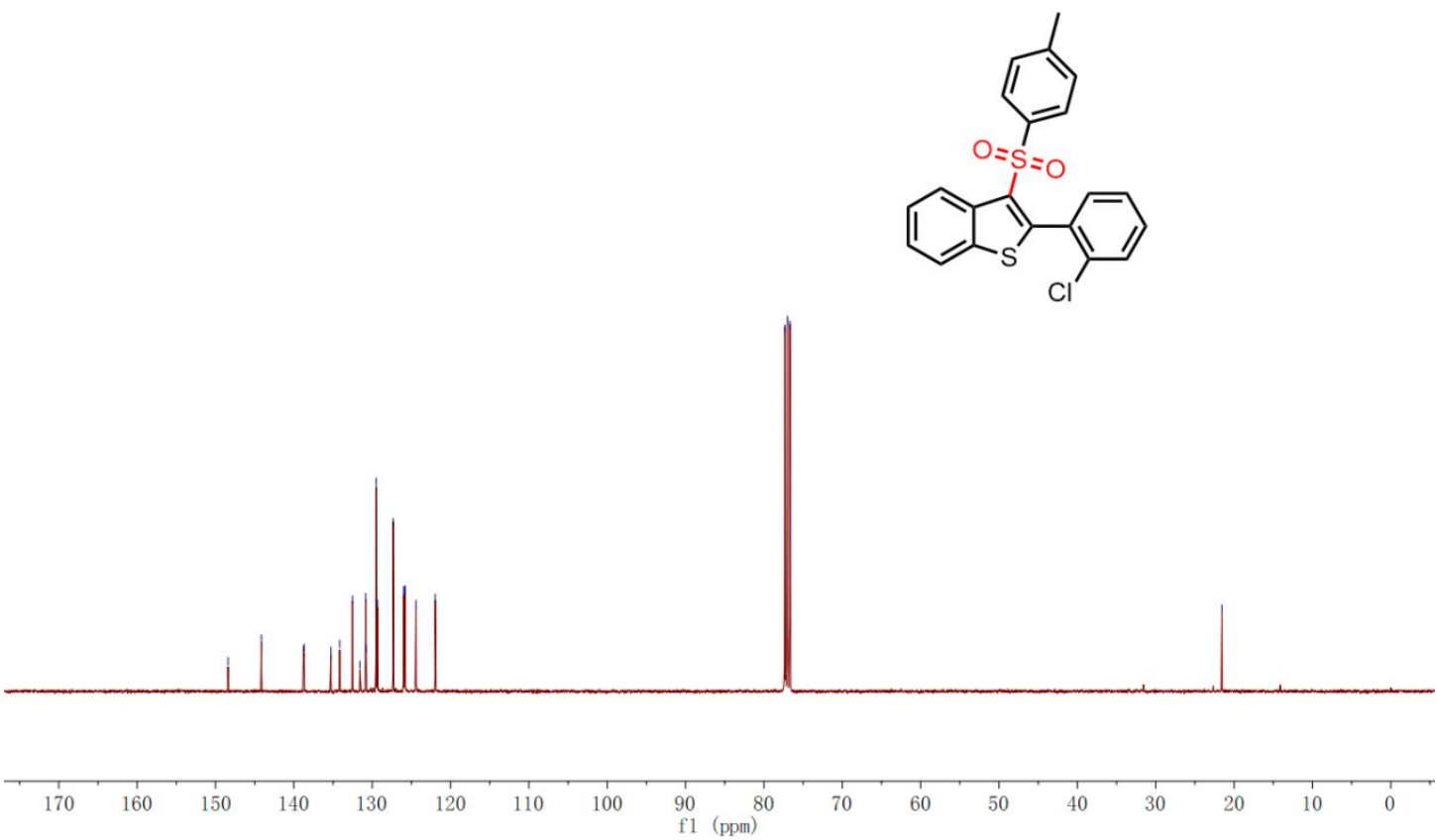

Figure S4. ${ }^{1} \mathrm{H}$ NMR of $\mathbf{3 d}\left(400 \mathrm{MHz}, \mathrm{CDCl}_{3}\right)$ and ${ }^{113} \mathrm{C}\left\{{ }^{1} \mathrm{H}\right\} \mathrm{NMR}$ of $\mathbf{3 d}\left(100 \mathrm{MHz}, \mathrm{CDCl}_{3}\right)$

2-(2-Bromophenyl)-3-tosylbenzo[b]thiophene (3e)

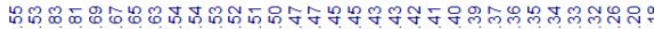

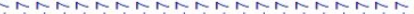
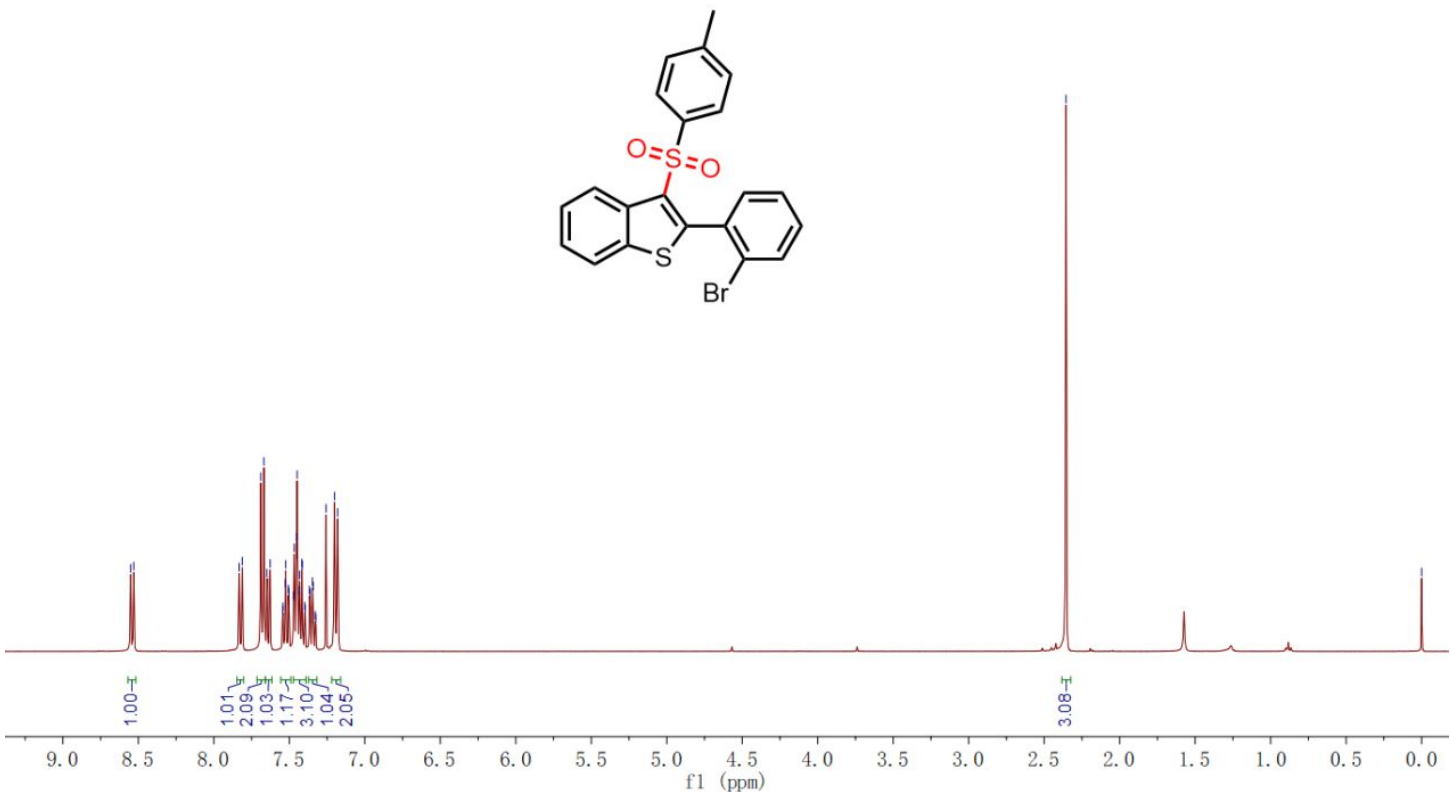

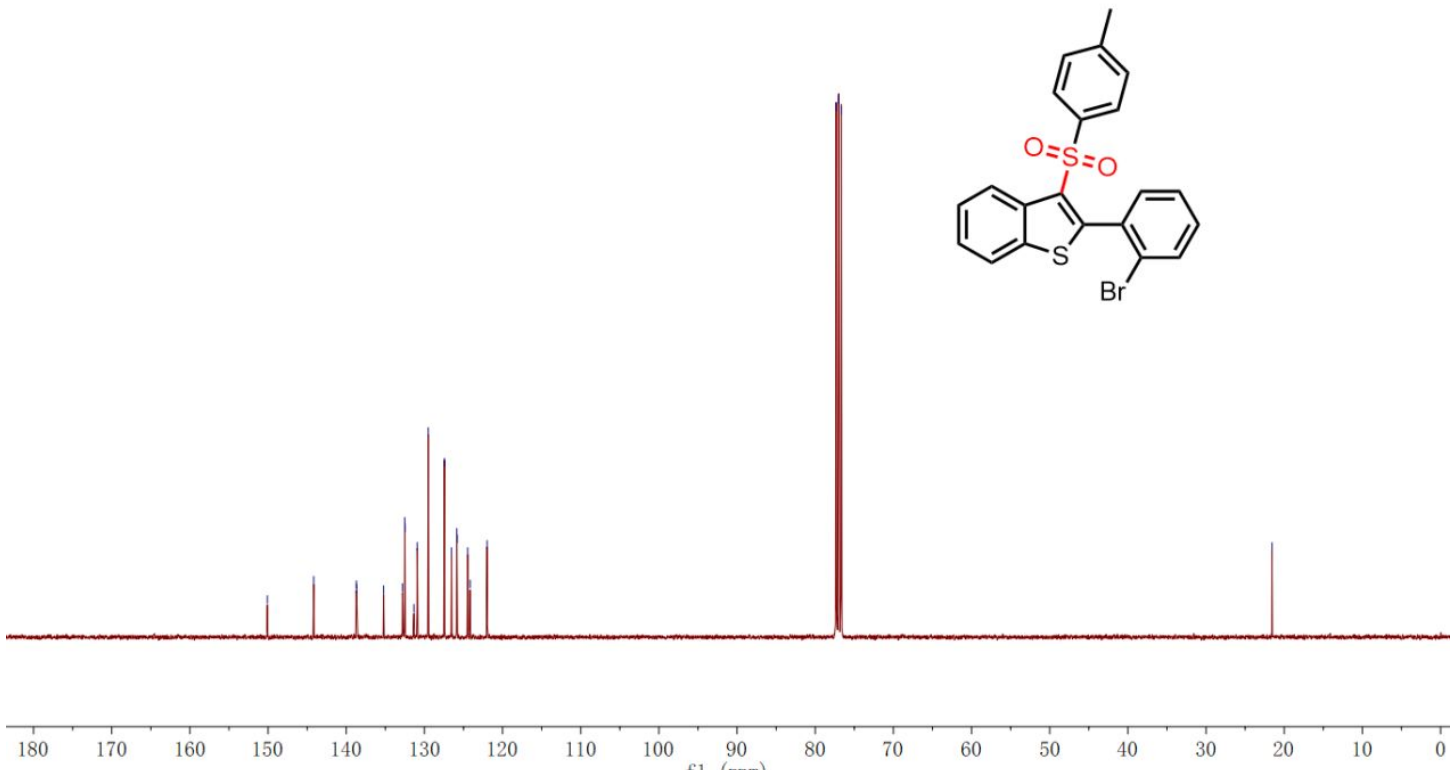

Figure S5. ${ }^{1} \mathrm{H}$ NMR of $3 \mathbf{e}\left(400 \mathrm{MHz}, \mathrm{CDCl}_{3}\right)$ and ${ }^{13} \mathrm{C}\left\{{ }^{1} \mathrm{H}\right\}$ NMR of $\mathbf{3 e}\left(100 \mathrm{MHz}, \mathrm{CDCl}_{3}\right)$

2-(m-Tolyl)-3-tosylbenzo[b]thiophene (3f)
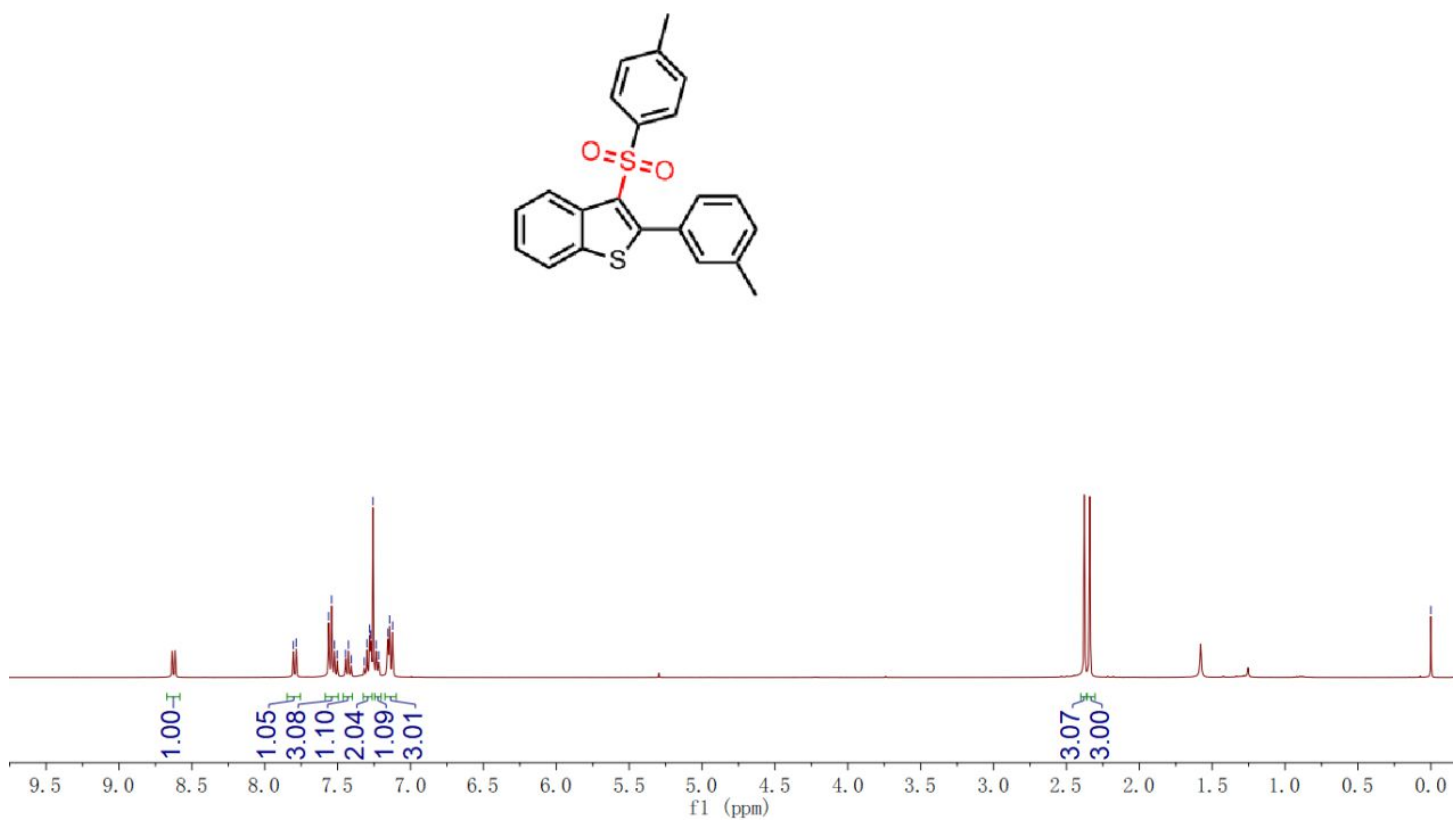

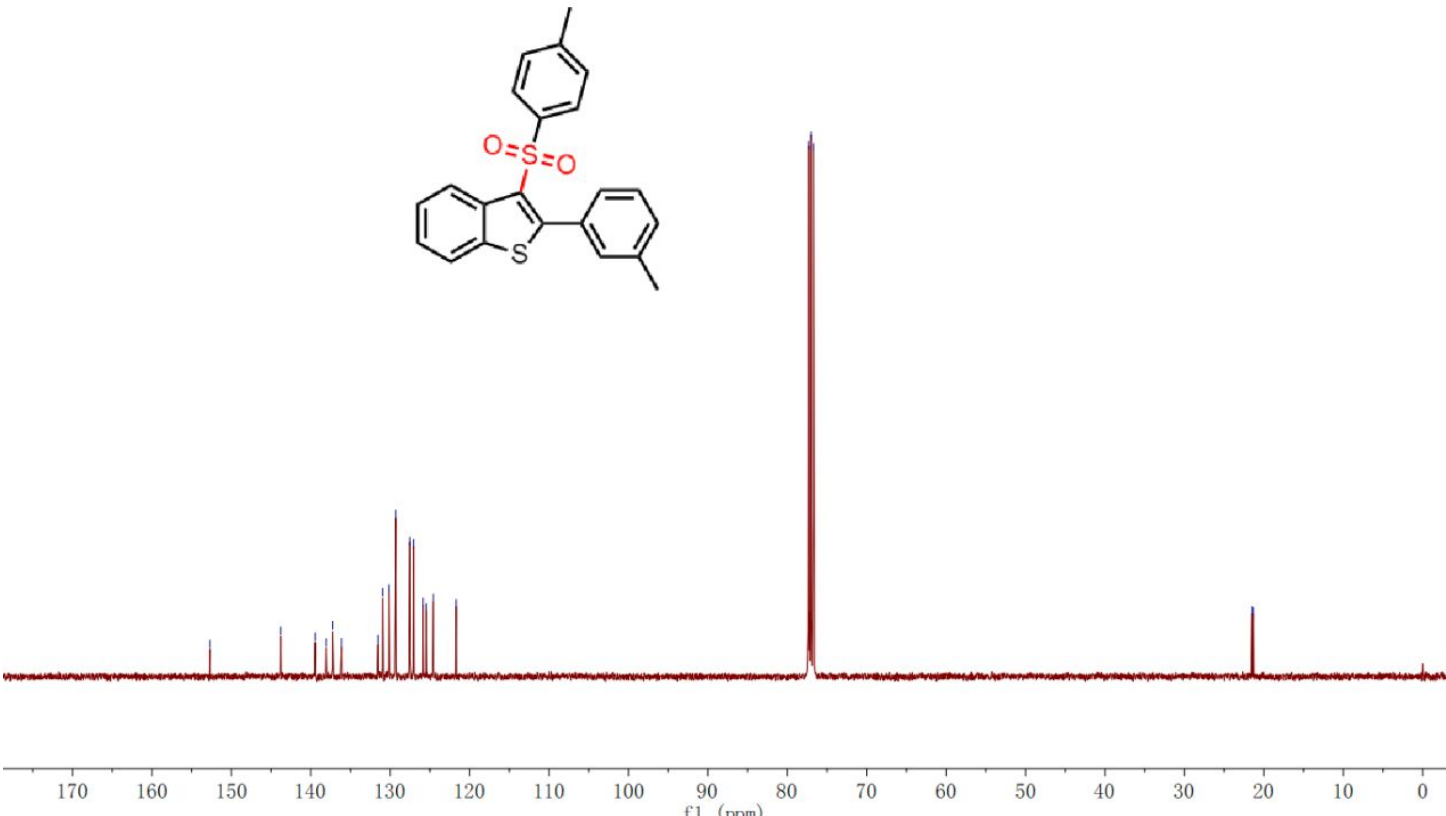

Figure S6. ${ }^{1} \mathrm{H}$ NMR of $\mathbf{3 f}\left(400 \mathrm{MHz}, \mathrm{CDCl}_{3}\right)$ and ${ }^{13} \mathrm{C}\left\{{ }^{1} \mathrm{H}\right\}$ NMR of $\mathbf{3 f}\left(100 \mathrm{MHz}, \mathrm{CDCl}_{3}\right)$

2-(3-Fluorophenyl)-3-tosylbenzo[b]thiophene (3g)

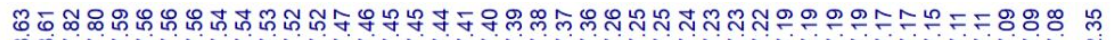

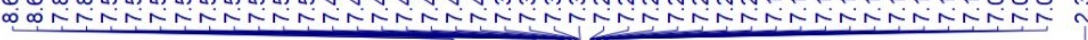<smiles>Cc1ccc(S(=O)(=O)c2c(-c3cccc(F)c3)sc3ccccc23)cc1</smiles>

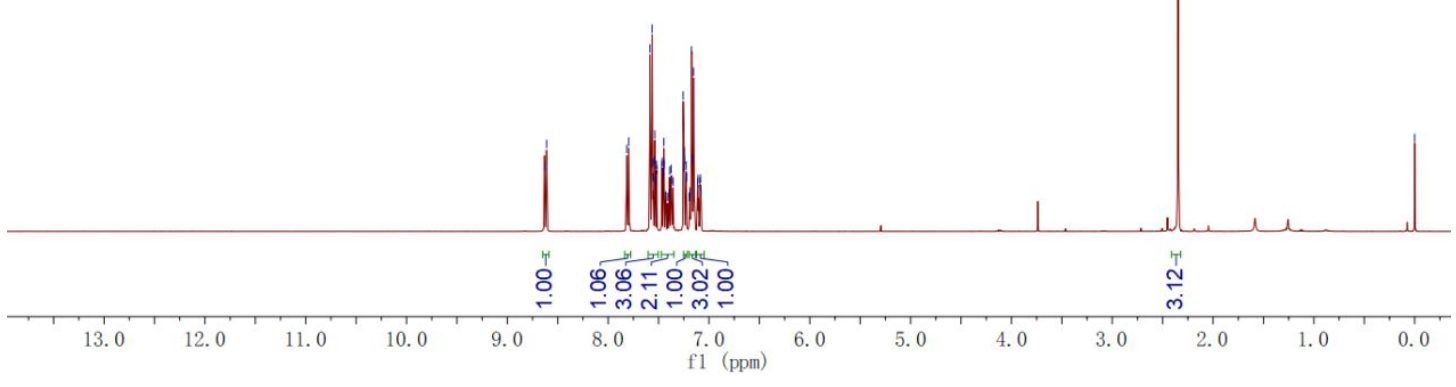



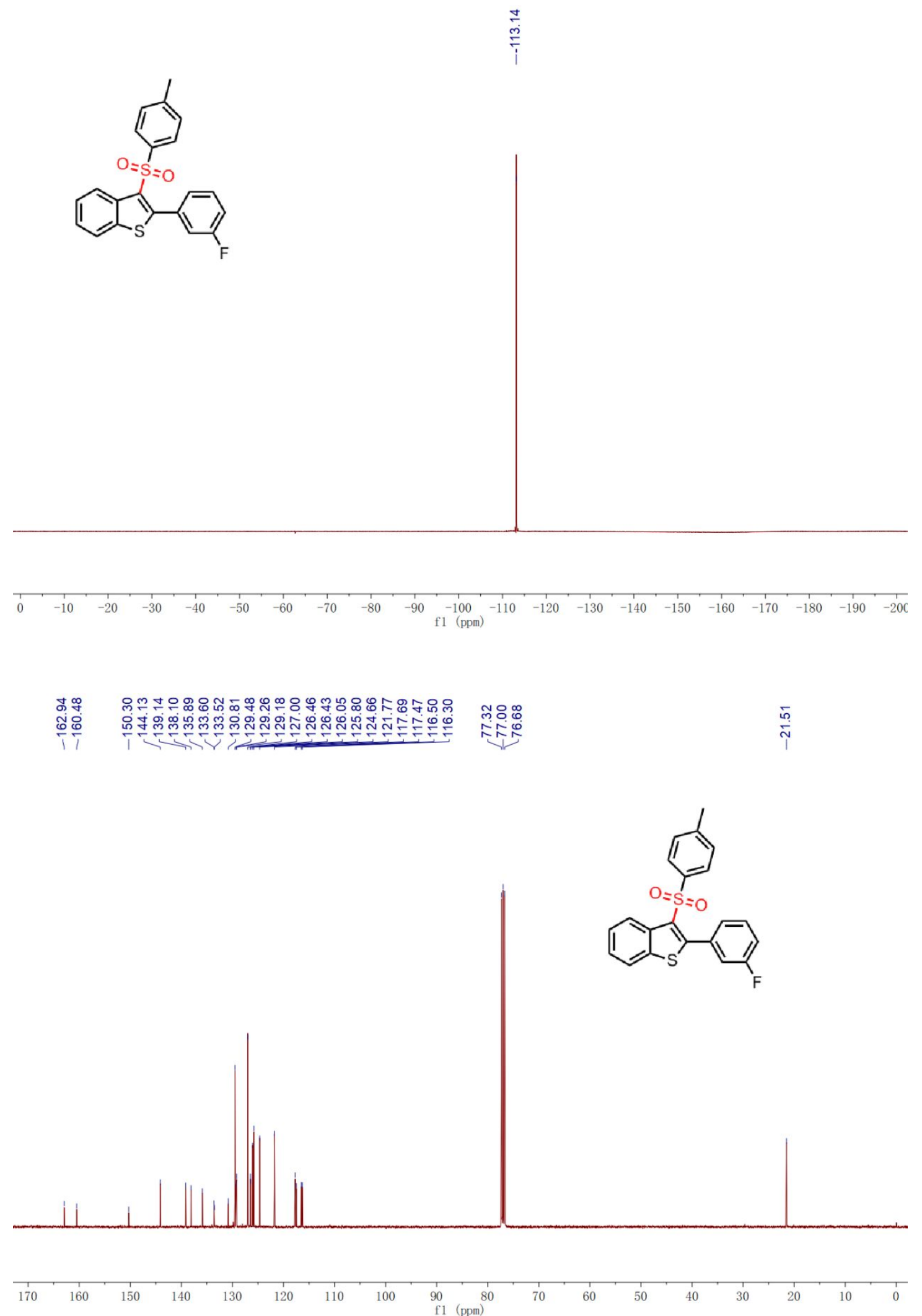

Figure S7. ${ }^{1} \mathrm{H}$ NMR of $\mathbf{3 g}\left(400 \mathrm{MHz}, \mathrm{CDCl}_{3}\right),{ }^{19} \mathrm{~F} \mathrm{NMR}$ of $\mathbf{3 g}\left(376 \mathrm{MHz}, \mathrm{CDCl}_{3}\right)$ and ${ }^{13} \mathrm{C}\left\{{ }^{1} \mathrm{H}\right\}$ NMR of $\mathbf{3 g}\left(100 \mathrm{MHz}, \mathrm{CDCl}_{3}\right)$ 
2-(3-Chlorophenyl)-3-tosylbenzo[b]thiophene (3h)

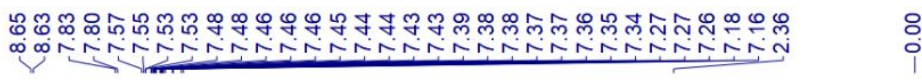<smiles>Cc1ccc(S(=O)(=O)c2c(-c3cccc(Cl)c3)sc3ccccc23)cc1</smiles>

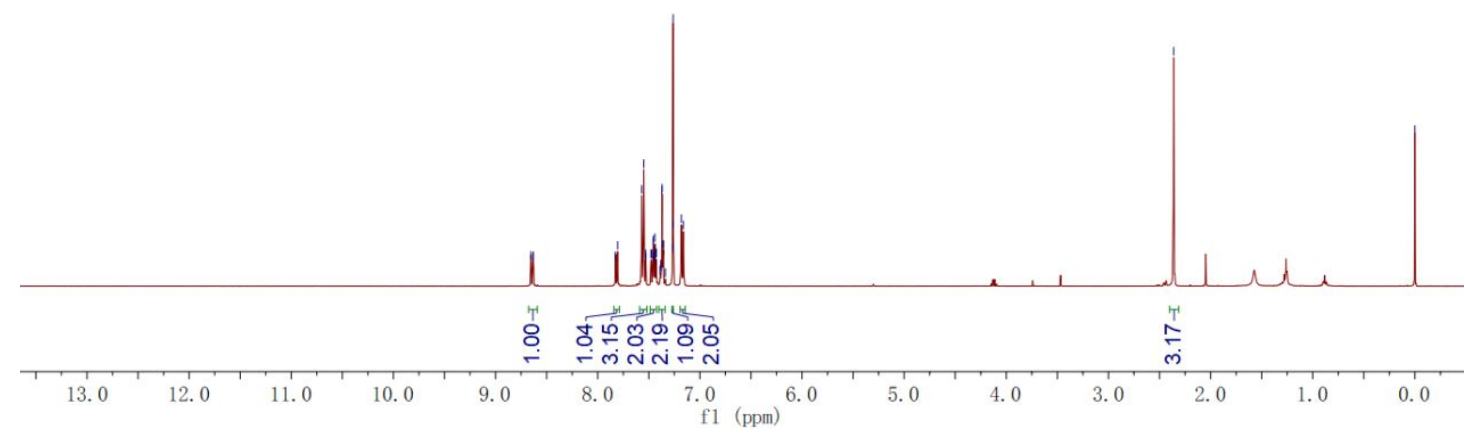

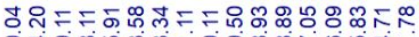

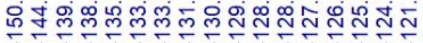

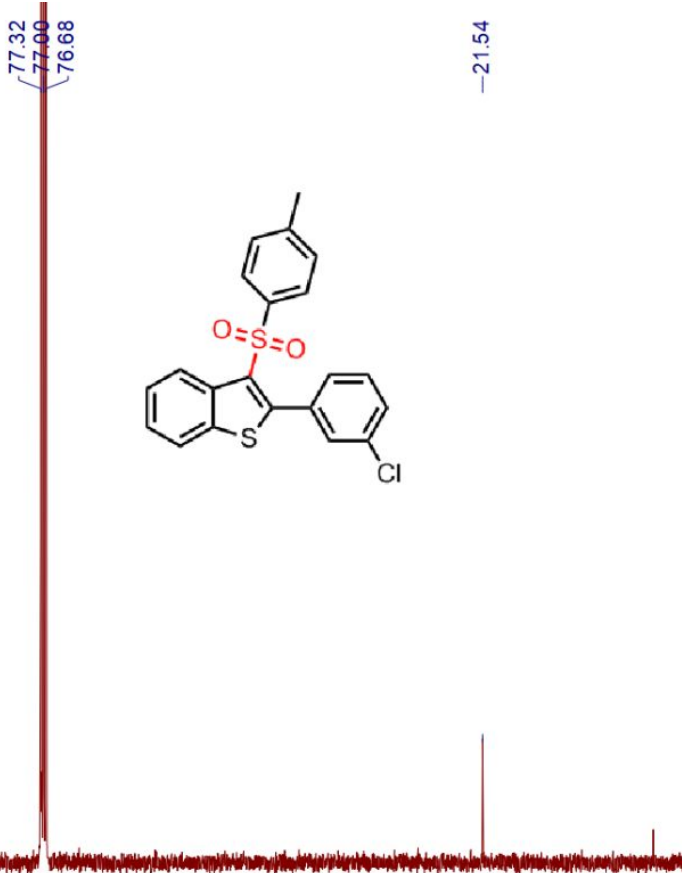

$\begin{array}{lllllllll}170 & 160 & 150 & 140 & 130 & 120 & 110 & 100 & 90 \\ \mathrm{fl}(\mathrm{ppm}) & 80\end{array}$

Figure S8. ${ }^{1} \mathrm{H}$ NMR of $\mathbf{3 h}\left(400 \mathrm{MHz}, \mathrm{CDCl}_{3}\right)$ and ${ }^{13} \mathrm{C}\left\{{ }^{1} \mathrm{H}\right\}$ NMR of $\mathbf{3 h}\left(100 \mathrm{MHz}, \mathrm{CDCl}_{3}\right)$ 


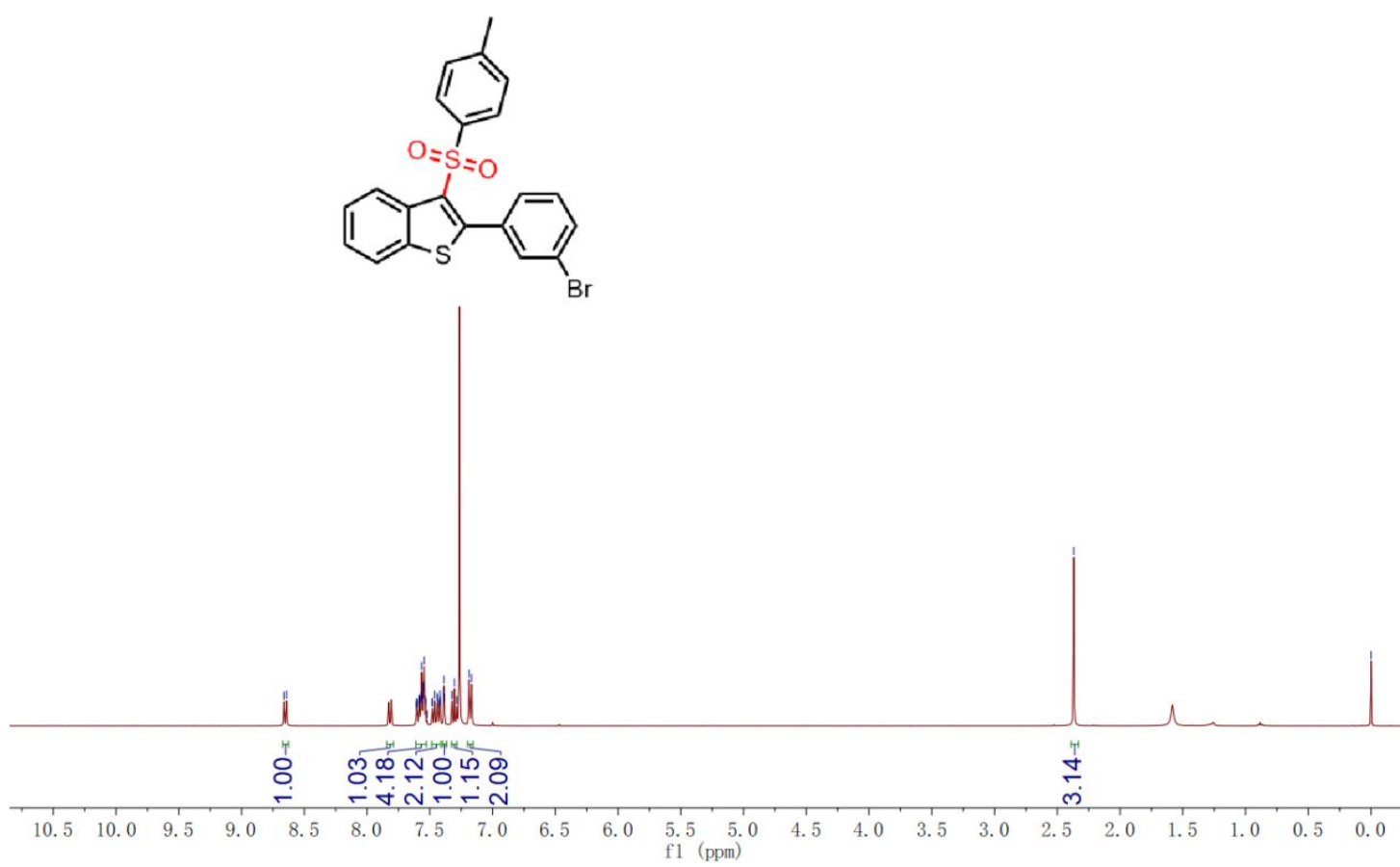<smiles>Cc1ccc(S(=O)(=O)c2c(-c3cccc(Br)c3)sc3ccccc23)cc1</smiles>

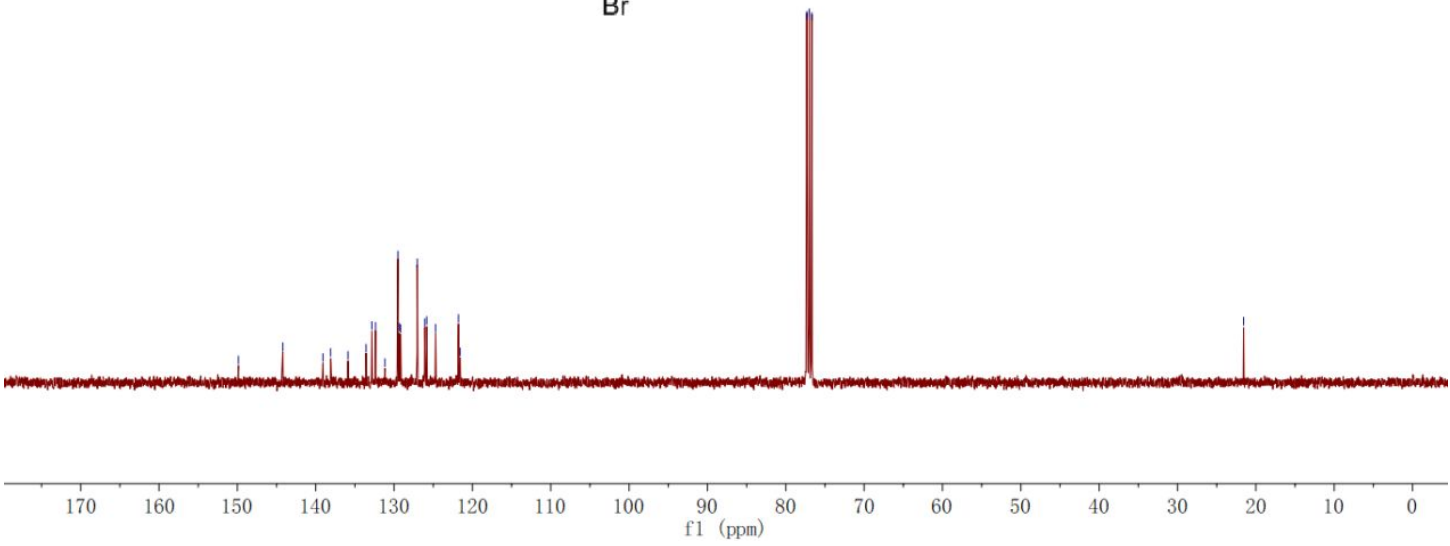

$\mathrm{Br}$

Figure S9. ${ }^{1} \mathrm{H}$ NMR of $3 \mathbf{i}\left(400 \mathrm{MHz}, \mathrm{CDCl}_{3}\right)$ and ${ }^{13} \mathrm{C}\left\{{ }^{1} \mathrm{H}\right\}$ NMR of $\mathbf{3 i}\left(100 \mathrm{MHz}, \mathrm{CDCl}_{3}\right)$ 
2-(p-tolyl)-3-tosylbenzo[b]thiophene (3j)

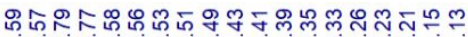

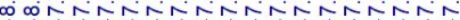

莳

웅
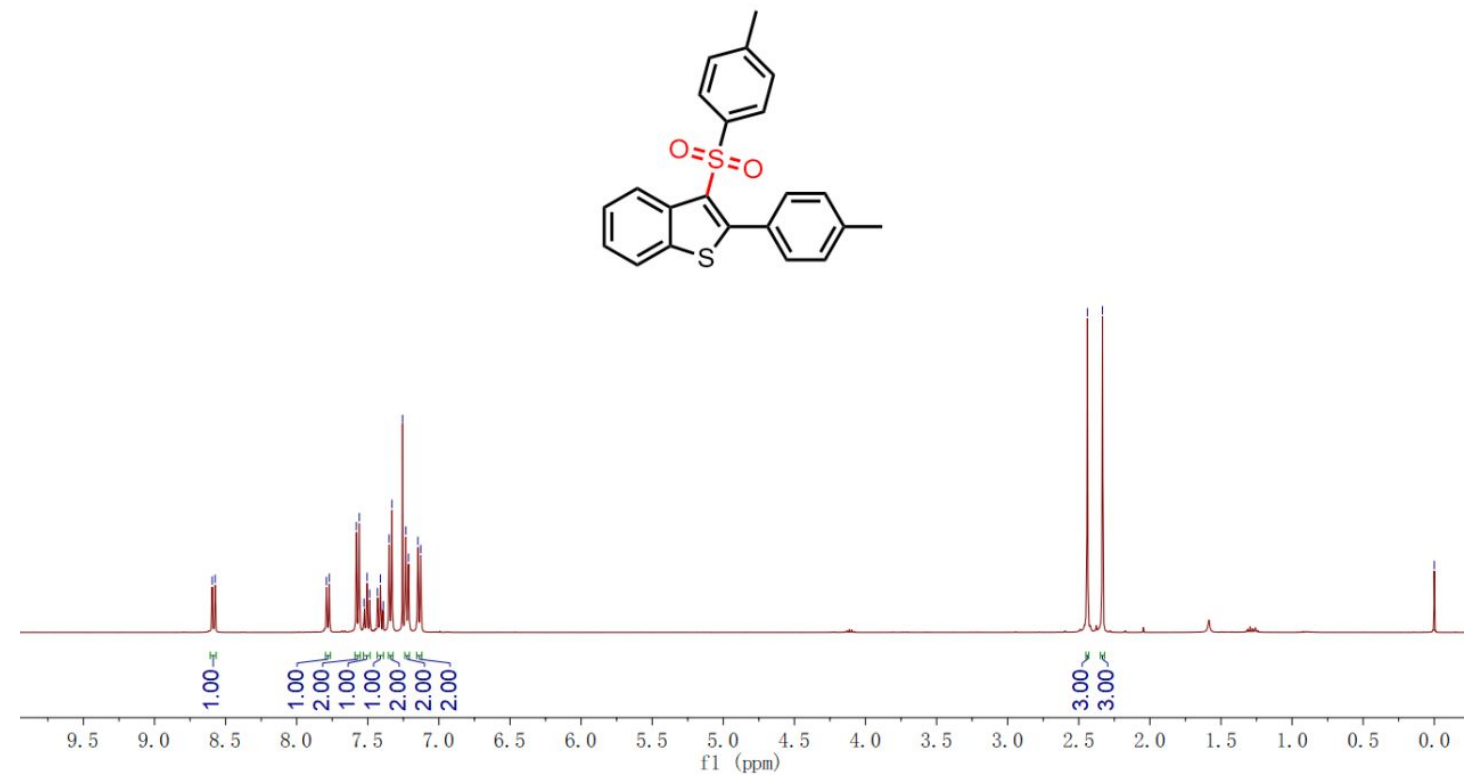

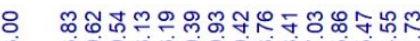

กิ
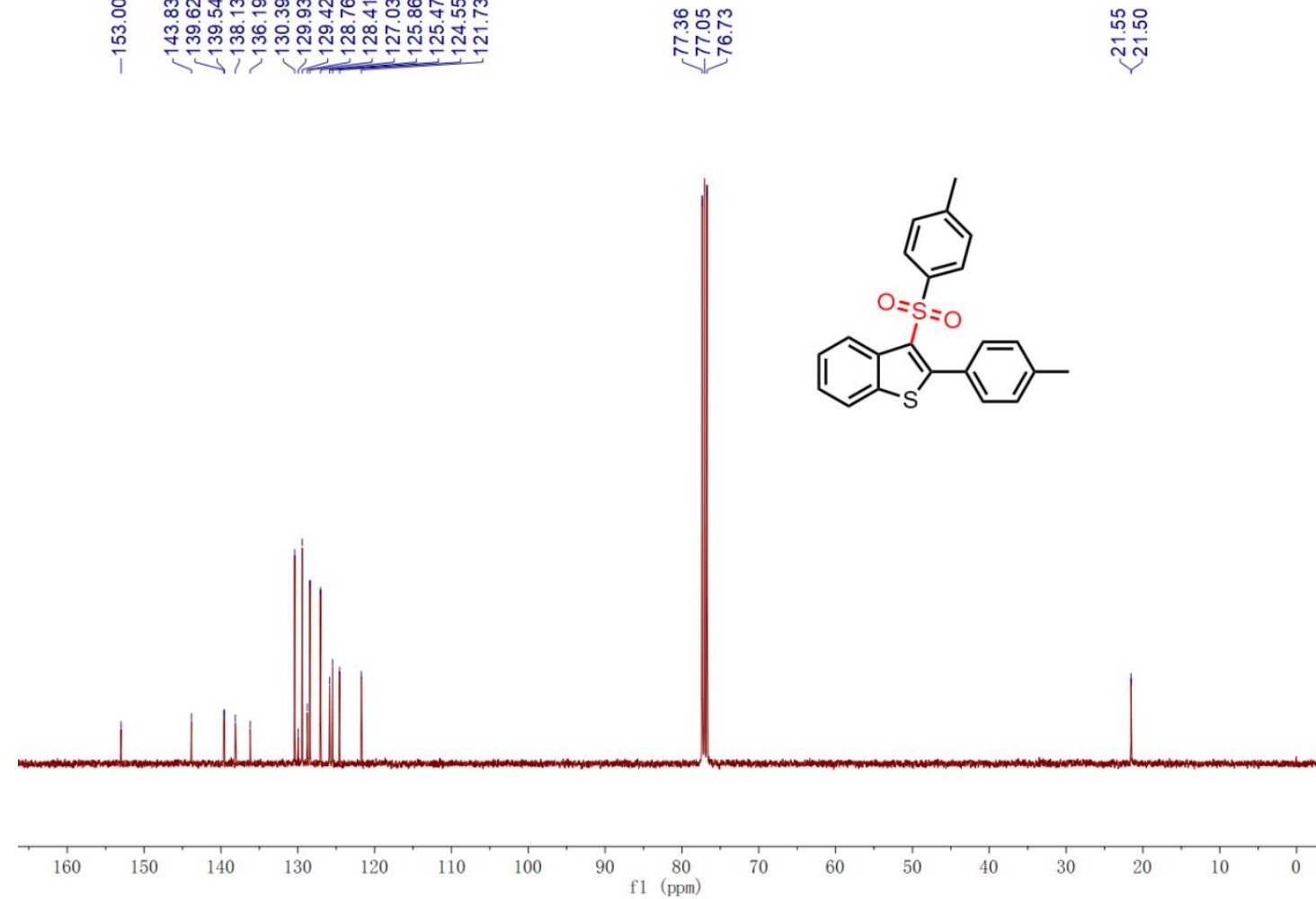

Figure S10. ${ }^{1} \mathrm{H}$ NMR of $\mathbf{3 j}\left(400 \mathrm{MHz}, \mathrm{CDCl}_{3}\right)$ and ${ }^{13} \mathrm{C}\left\{{ }^{1} \mathrm{H}\right\}$ NMR of $\mathbf{3 j}\left(100 \mathrm{MHz}, \mathrm{CDCl}_{3}\right)$ 
2-(4-Ethylphenyl)-3-tosylbenzo[b]thiophene (3k)

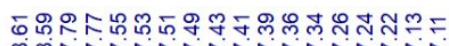

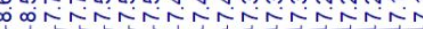

ㅅN슈ำ

:
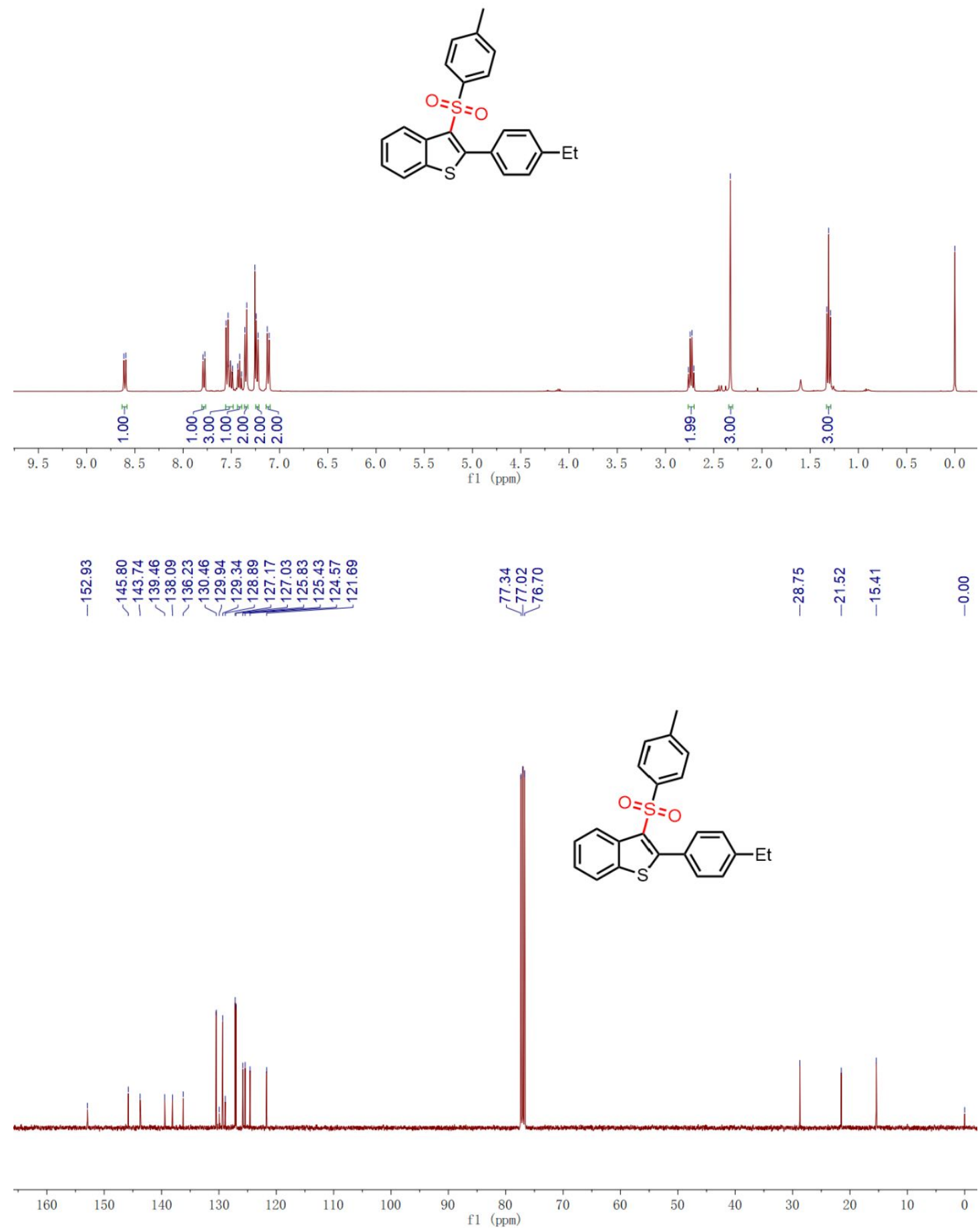

Figure S11. ${ }^{1} \mathrm{H}$ NMR of $\mathbf{3 k}\left(400 \mathrm{MHz}, \mathrm{CDCl}_{3}\right)$ and ${ }^{13} \mathrm{C}\left\{{ }^{1} \mathrm{H}\right\}$ NMR of $\mathbf{3 k}\left(100 \mathrm{MHz}, \mathrm{CDCl}_{3}\right)$ 
2-(4-Methoxyphenyl)-3-tosylbenzo[b]thiophene (3I)

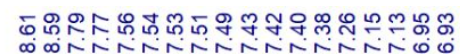
o

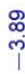

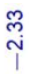
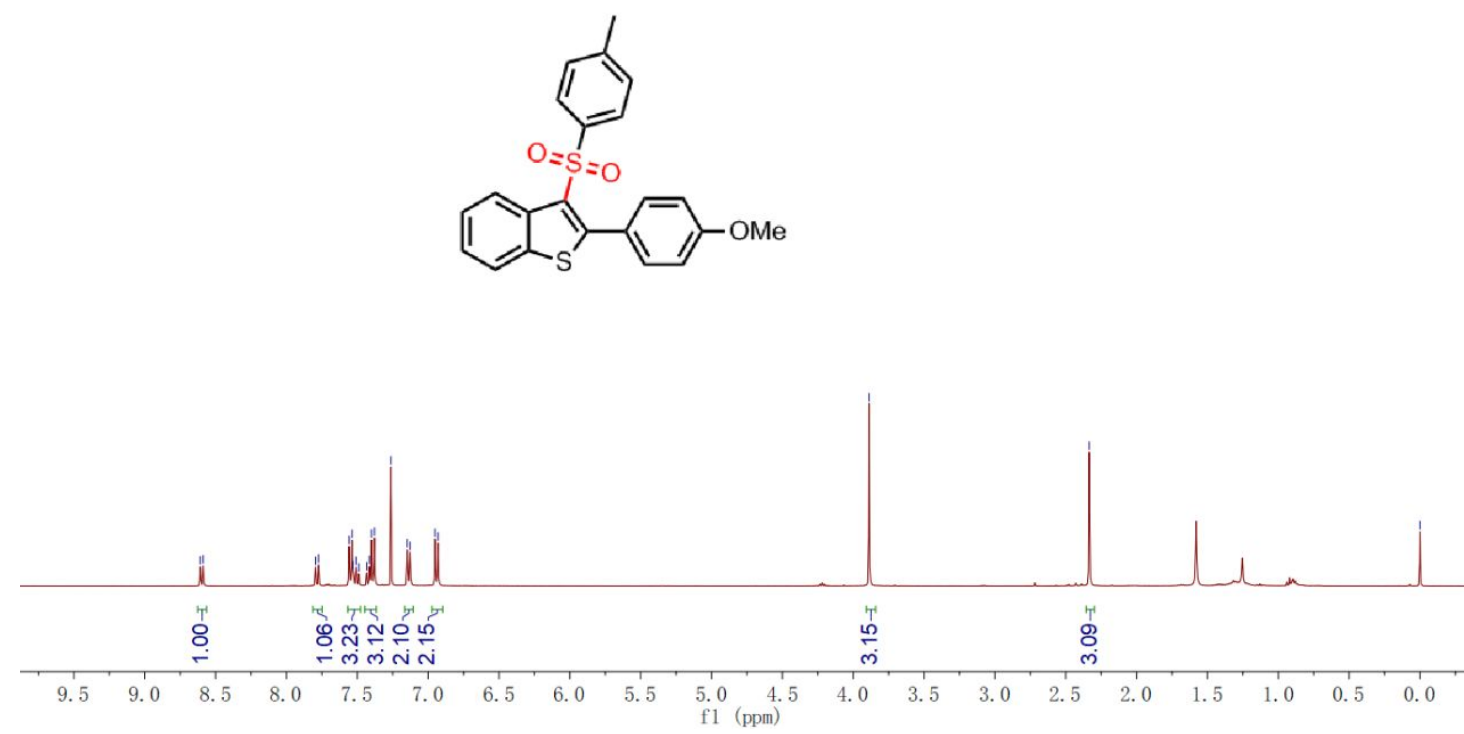

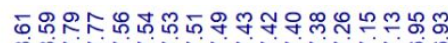

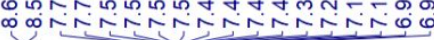

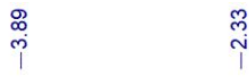

:
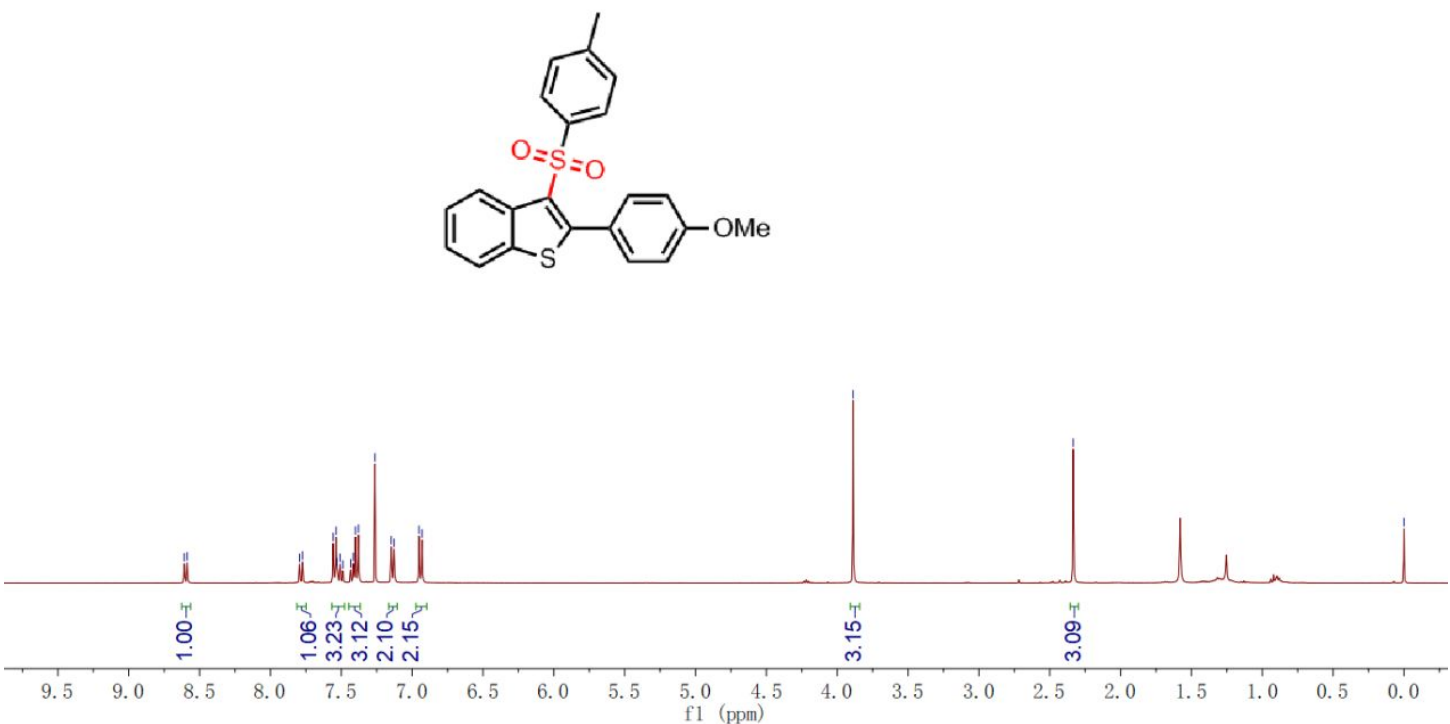

Figure S12. ${ }^{1} \mathrm{H}$ NMR of $31\left(400 \mathrm{MHz}, \mathrm{CDCl}_{3}\right)$ and ${ }^{13} \mathrm{C}\left\{{ }^{1} \mathrm{H}\right\} \mathrm{NMR}$ of $31\left(100 \mathrm{MHz}, \mathrm{CDCl}_{3}\right)$ 
2-(4-Fluorophenyl)-3-tosylbenzo[b]thiophene (3 m)

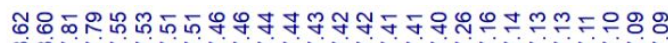
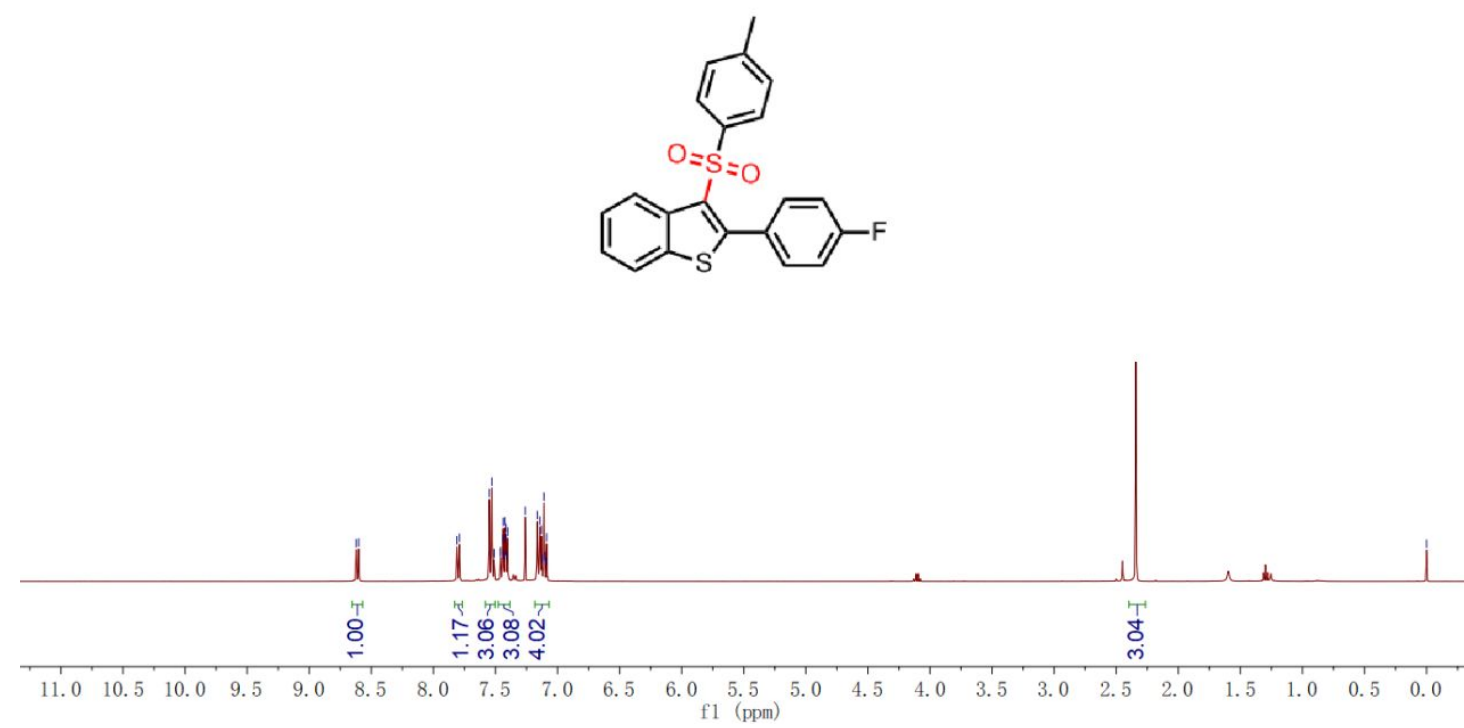

$\stackrel{\stackrel{7}{i}}{\stackrel{i}{i}}$
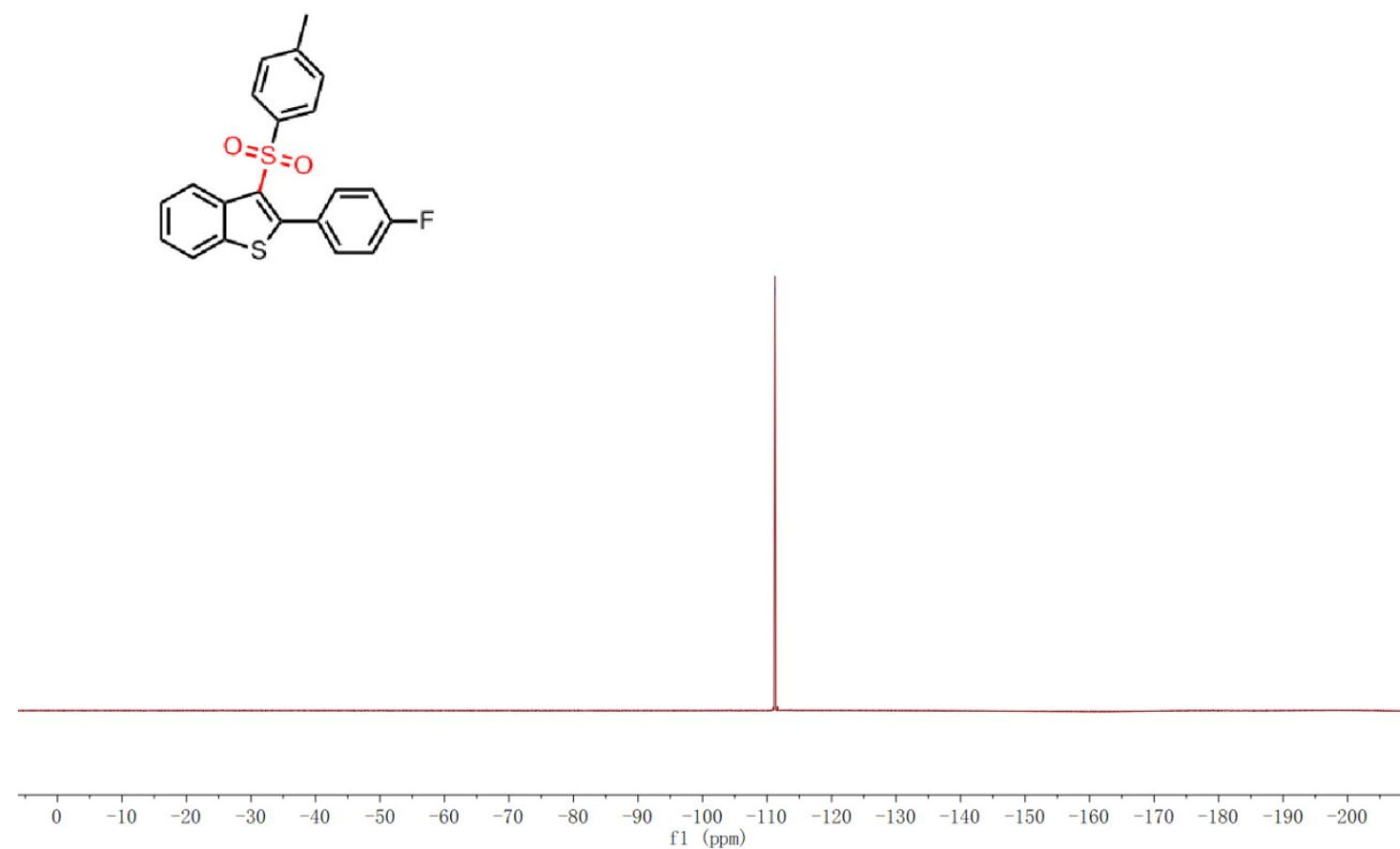

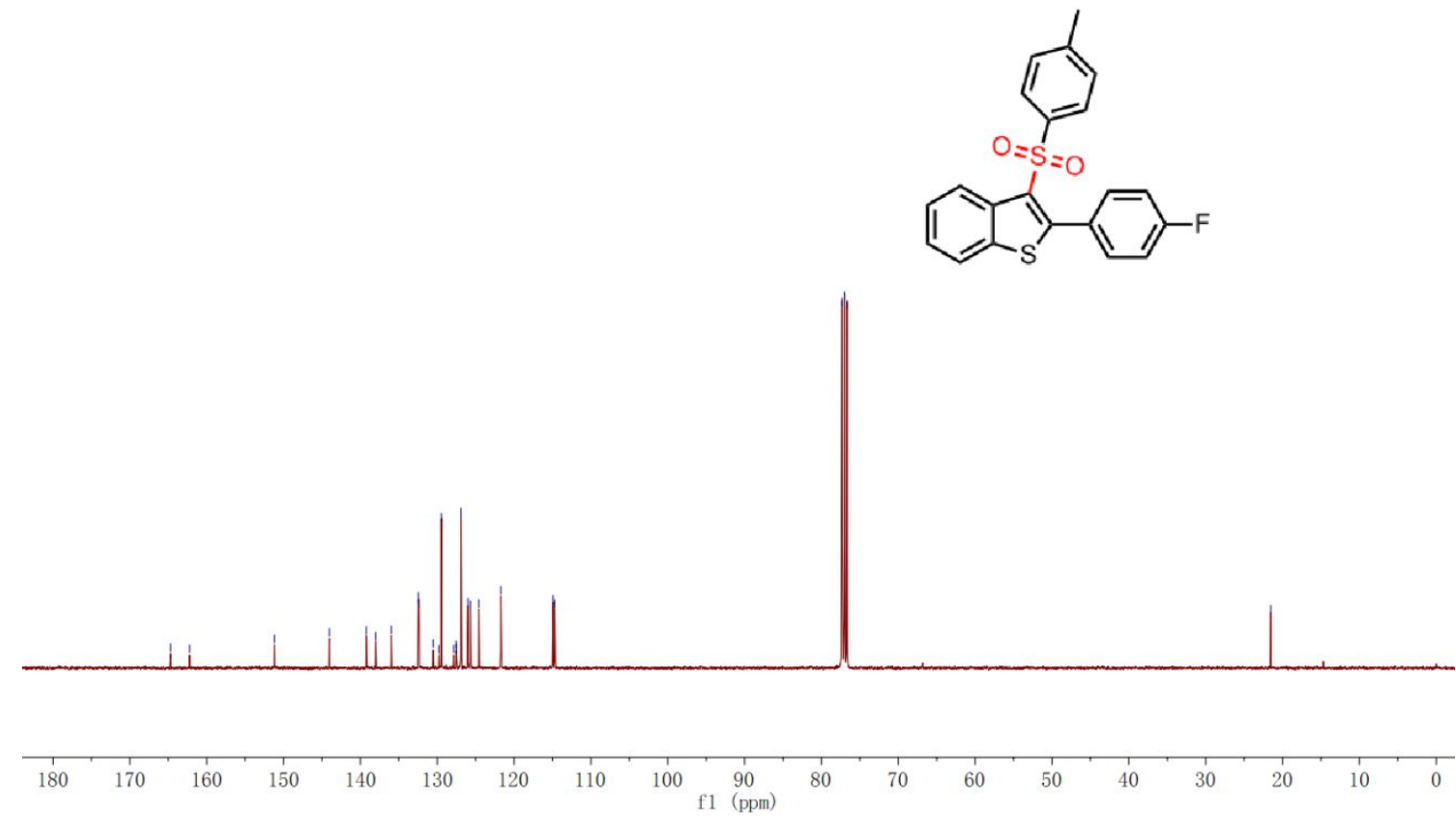

Figure S13. ${ }^{1} \mathrm{H}$ NMR of $\mathbf{3 m}\left(400 \mathrm{MHz}, \mathrm{CDCl}_{3}\right),{ }^{19} \mathrm{~F} \mathrm{NMR}$ of $\mathbf{3 g}\left(376 \mathrm{MHz}, \mathrm{CDCl}_{3}\right)$ and ${ }^{13} \mathrm{C}\left\{{ }^{1} \mathrm{H}\right\}$ NMR of $3 \mathbf{m}\left(100 \mathrm{MHz}, \mathrm{CDCl}_{3}\right)$

2-(4-Chlorophenyl)-3-tosylbenzo[b]thiophene (3n)

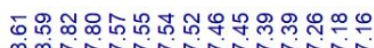

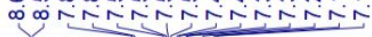
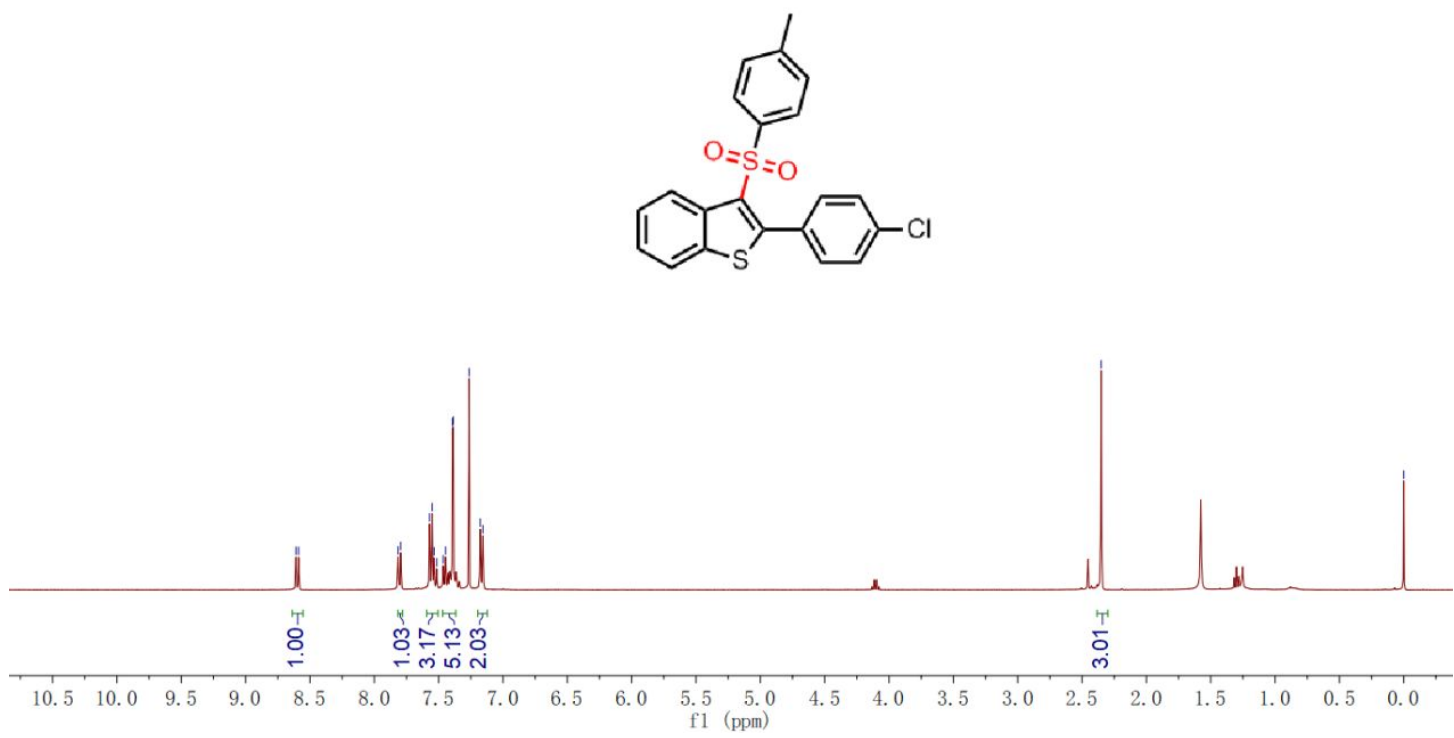


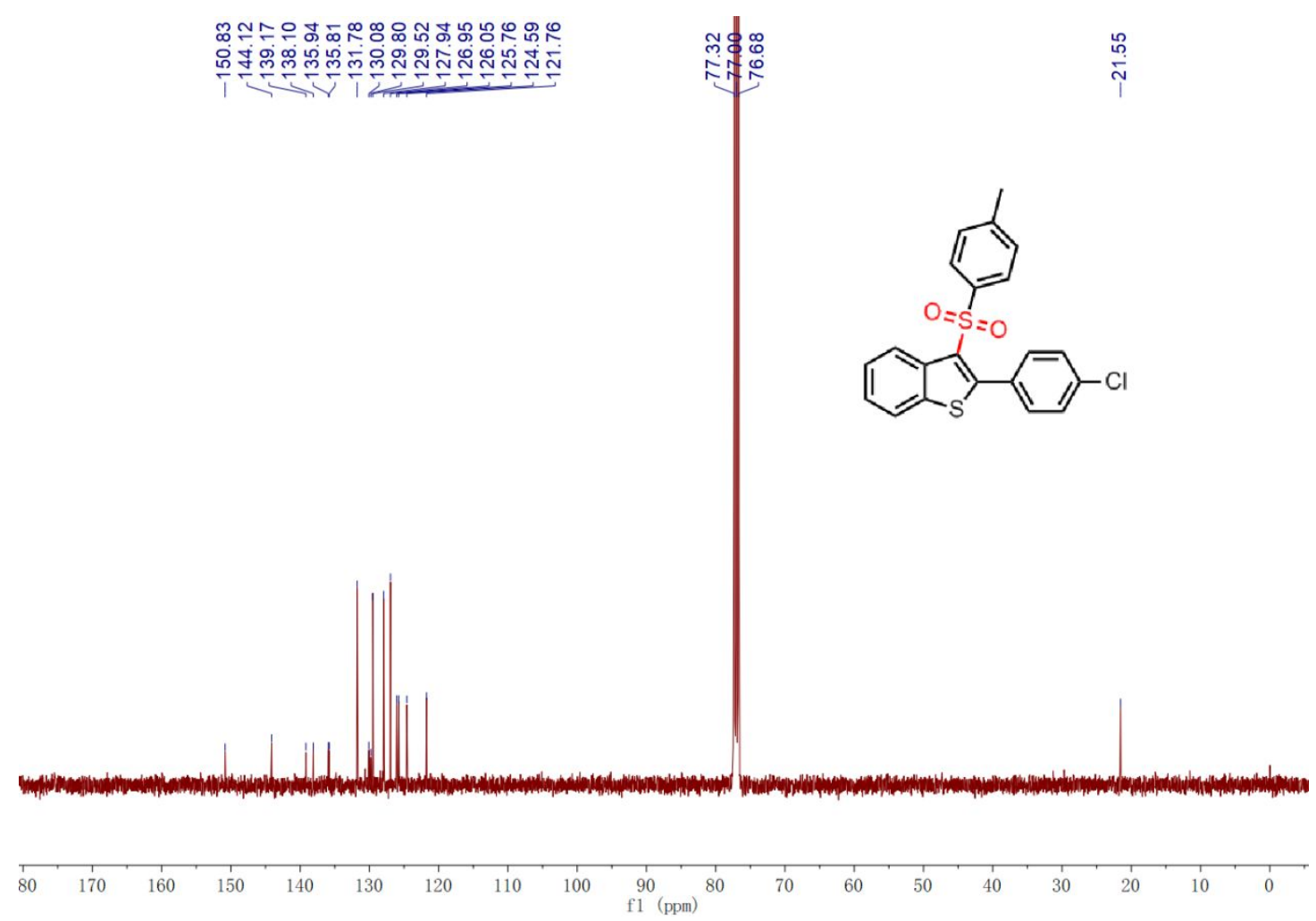

Figure S14. ${ }^{1} \mathrm{H}$ NMR of $3 \mathbf{n}\left(400 \mathrm{MHz}, \mathrm{CDCl}_{3}\right)$ and ${ }^{13} \mathrm{C}\left\{{ }^{1} \mathrm{H}\right\}$ NMR of $\mathbf{3 n}\left(100 \mathrm{MHz}, \mathrm{CDCl}_{3}\right)$

2-(4-Bromophenyl)-3-tosylbenzo[b]thiophene (3o)

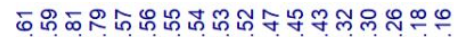

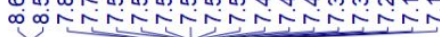
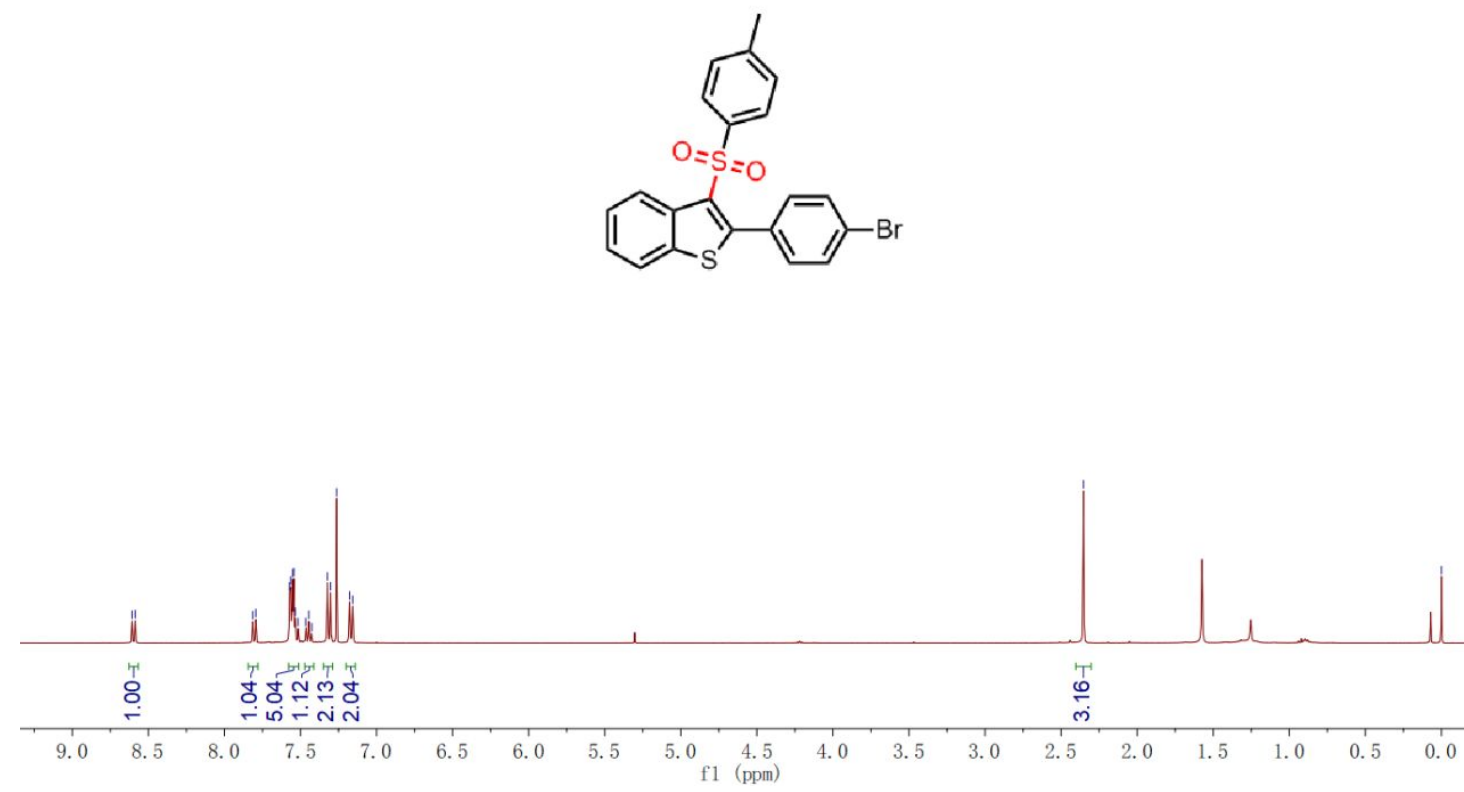


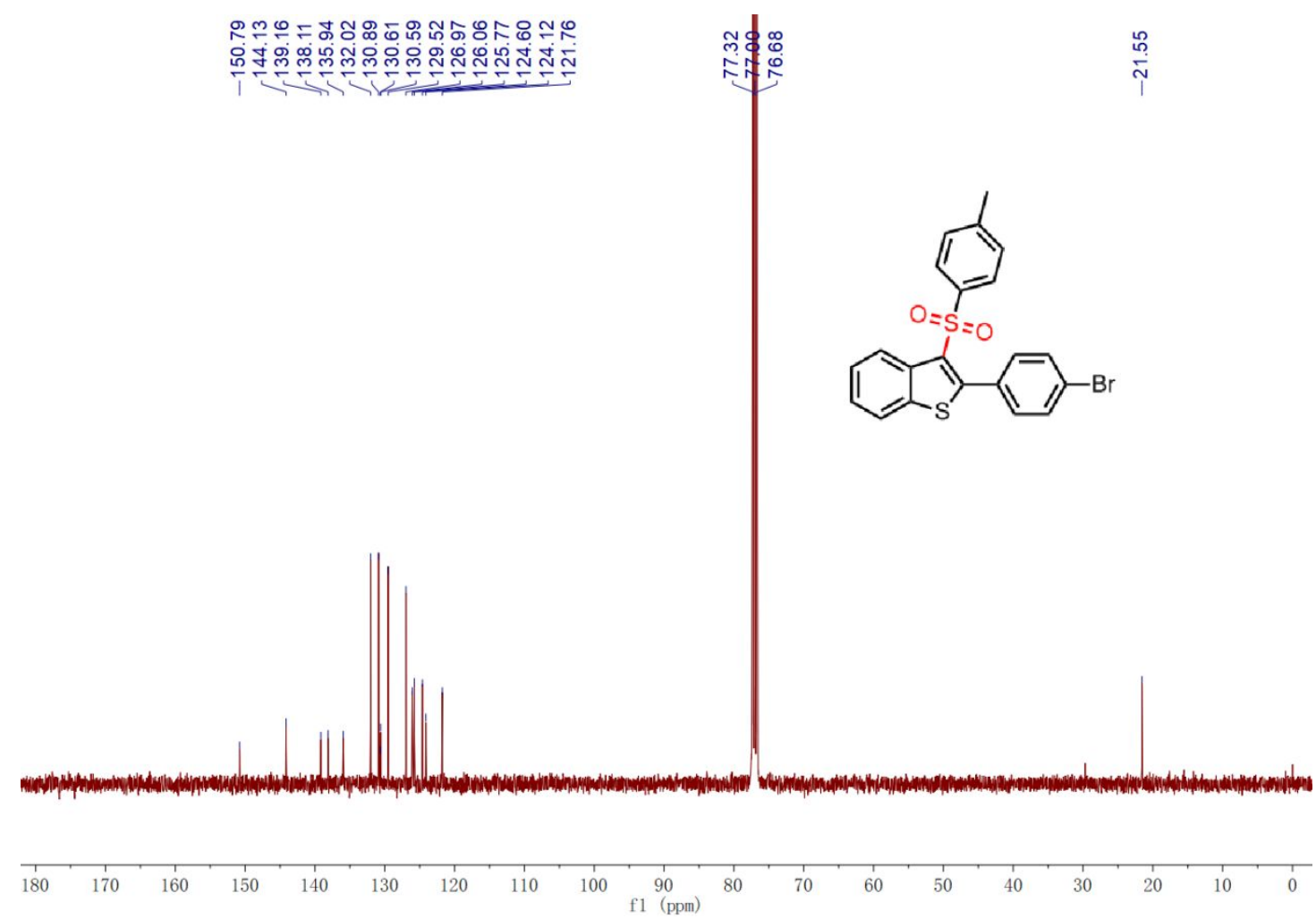

Figure S15. ${ }^{1} \mathrm{H}$ NMR of $30\left(400 \mathrm{MHz}, \mathrm{CDCl}_{3}\right)$ and ${ }^{13} \mathrm{C}\left\{{ }^{1} \mathrm{H}\right\} \mathrm{NMR}$ of $\mathbf{3 o}\left(100 \mathrm{MHz}, \mathrm{CDCl}_{3}\right)$

Methyl 4-(3-tosylbenzo[b]thiophen-2-yl) benzoate (3p)

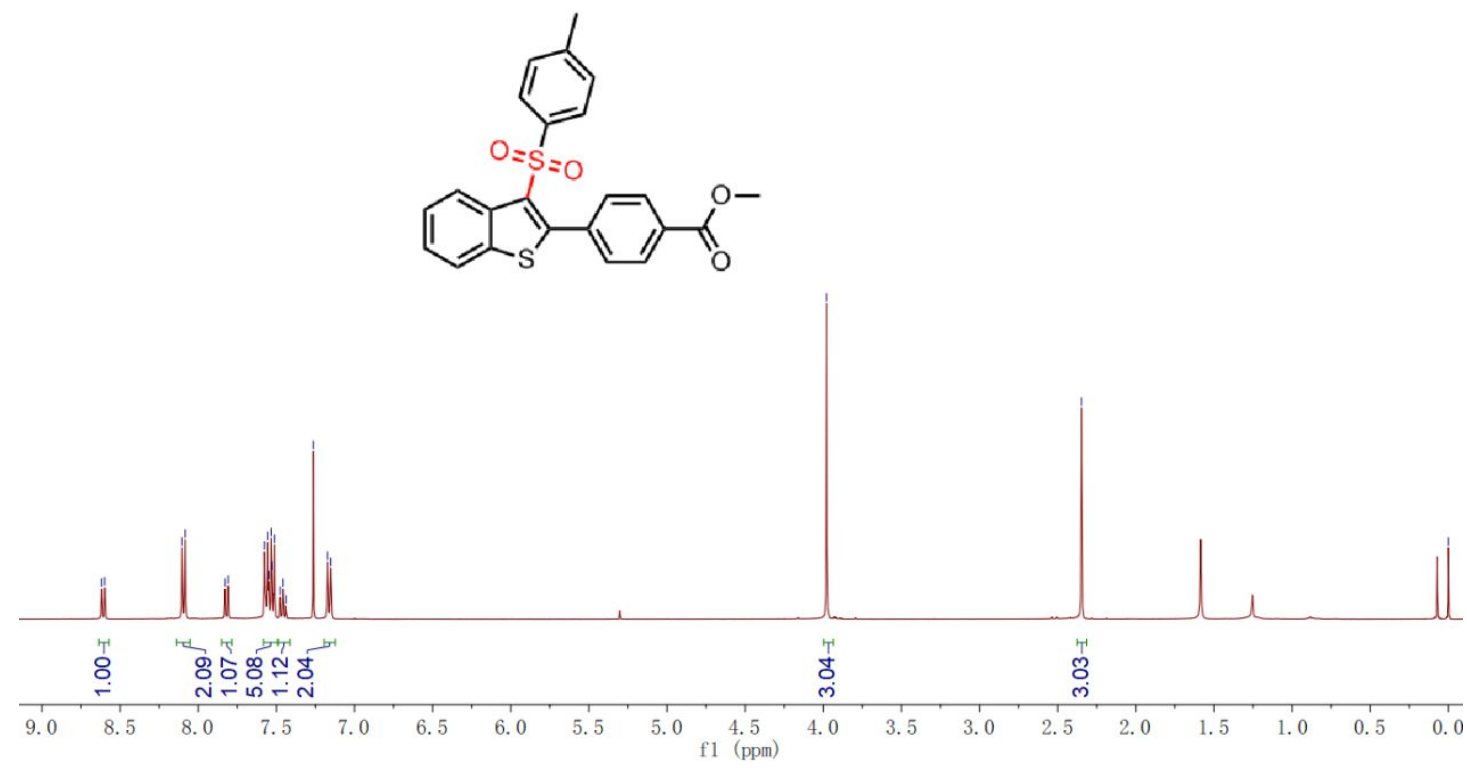




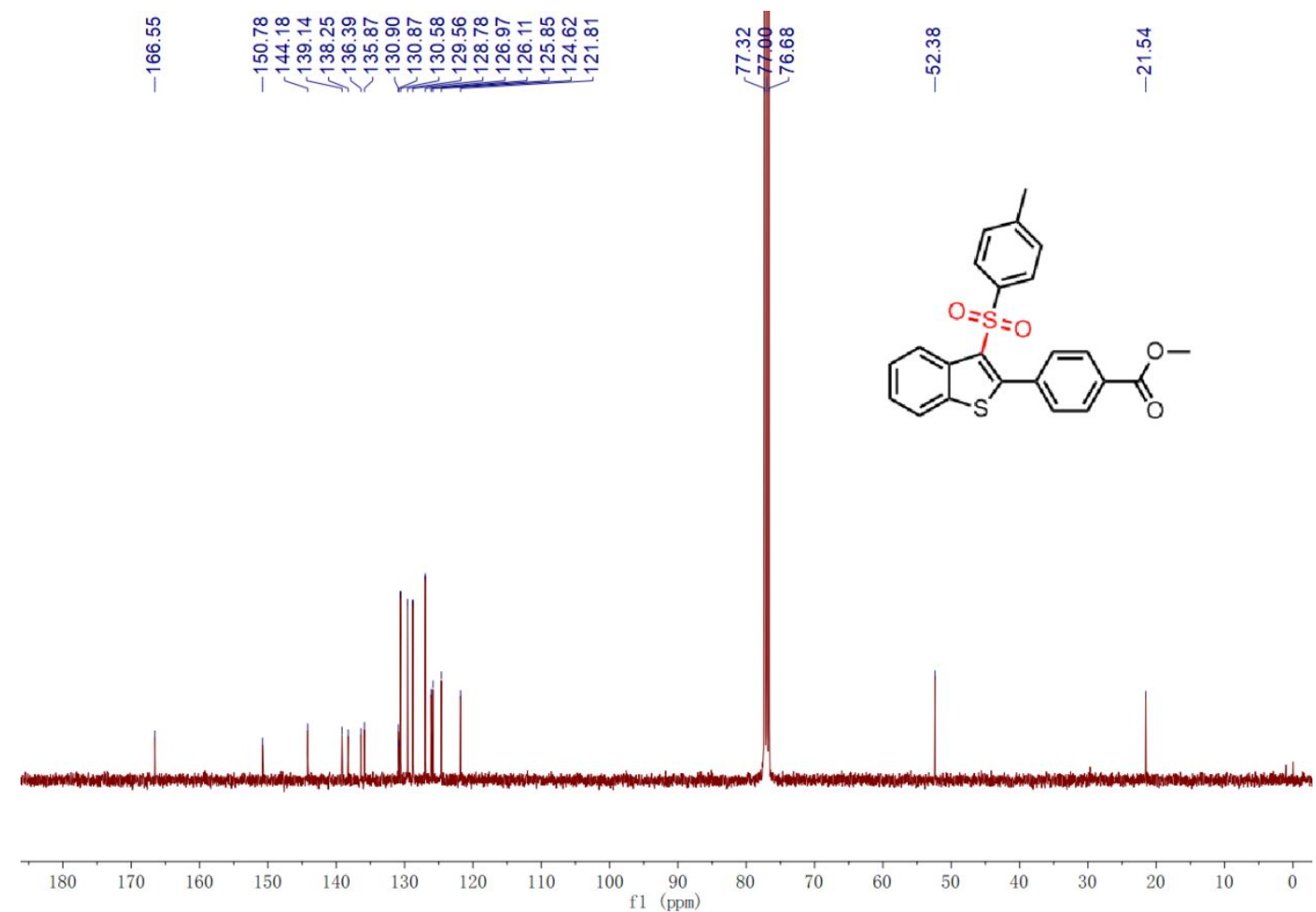

Figure S16. ${ }^{1} \mathrm{H}$ NMR of $\mathbf{3 p}\left(400 \mathrm{MHz}, \mathrm{CDCl}_{3}\right)$ and ${ }^{13} \mathrm{C}\left\{{ }^{1} \mathrm{H}\right\}$ NMR of $\mathbf{3 p}\left(100 \mathrm{MHz}, \mathrm{CDCl}_{3}\right)$

3-Tosyl-2-(4-(trifluoromethyl) phenyl) benzo[b]thiophene (3q)

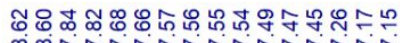

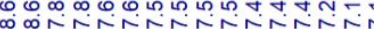
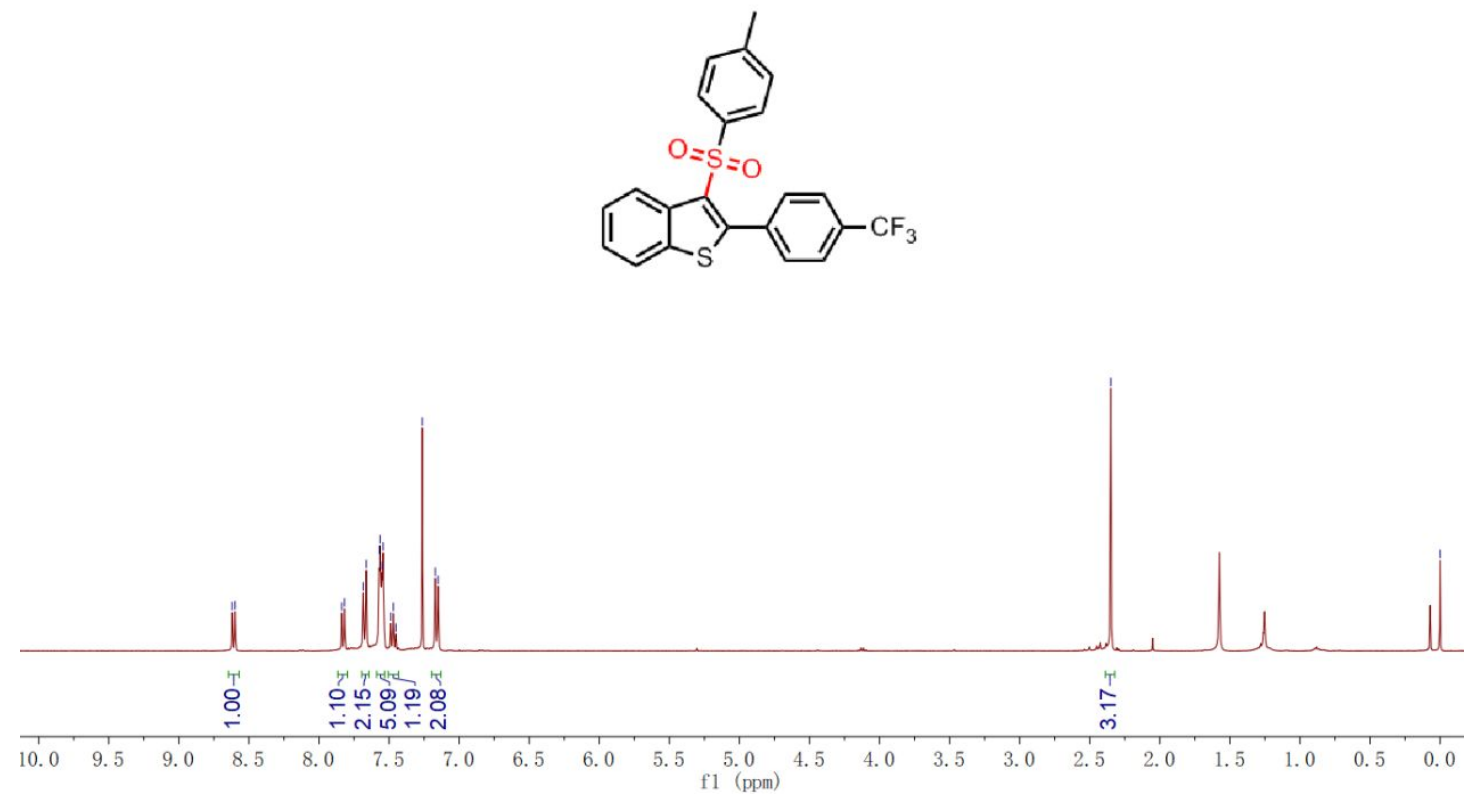

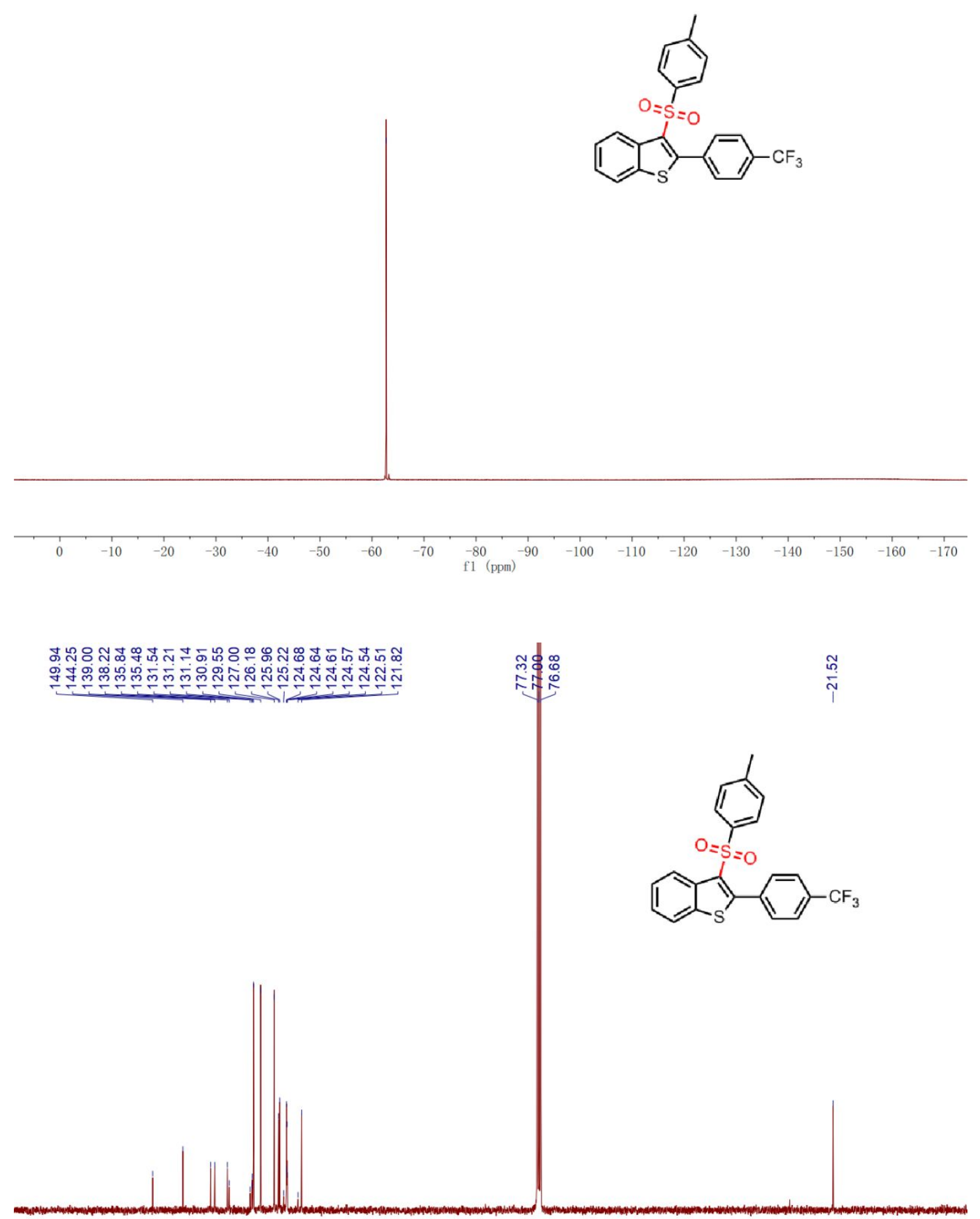

$\begin{array}{llllllllllllllllll}170 & 160 & 150 & 140 & 130 & 120 & 110 & 100 & \begin{array}{l}1 \\ \mathrm{f} 1\end{array}(\mathrm{ppm}) & 80 & 70 & 60 & 50 & 40 & 30 & 20 & 10 & 1\end{array}$

Figure S17. ${ }^{1} \mathrm{H} \mathrm{NMR}$ of 3q $\left(400 \mathrm{MHz}, \mathrm{CDCl}_{3}\right),{ }^{19} \mathrm{~F} \mathrm{NMR} \mathrm{of} \mathbf{3 q}\left(376 \mathrm{MHz}, \mathrm{CDCl}_{3}\right)$ and ${ }^{13} \mathrm{C}\left\{{ }^{1} \mathrm{H}\right\}$ NMR of 3q $\left(100 \mathrm{MHz}, \mathrm{CDCl}_{3}\right)$ 
2-(Thiophen-2-yl)-3-tosylbenzo[b]thiophene (3r)
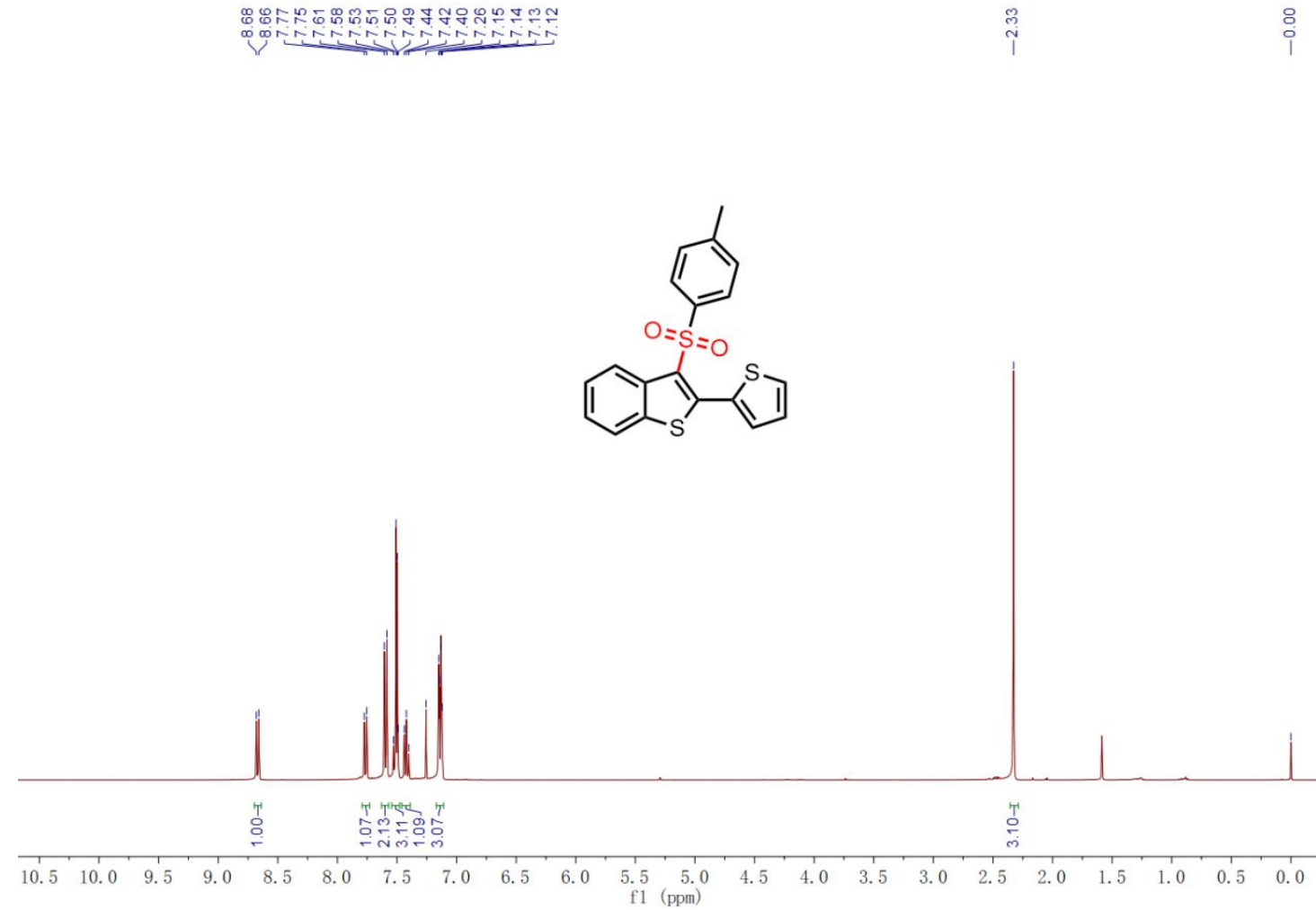

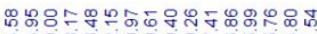

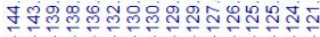
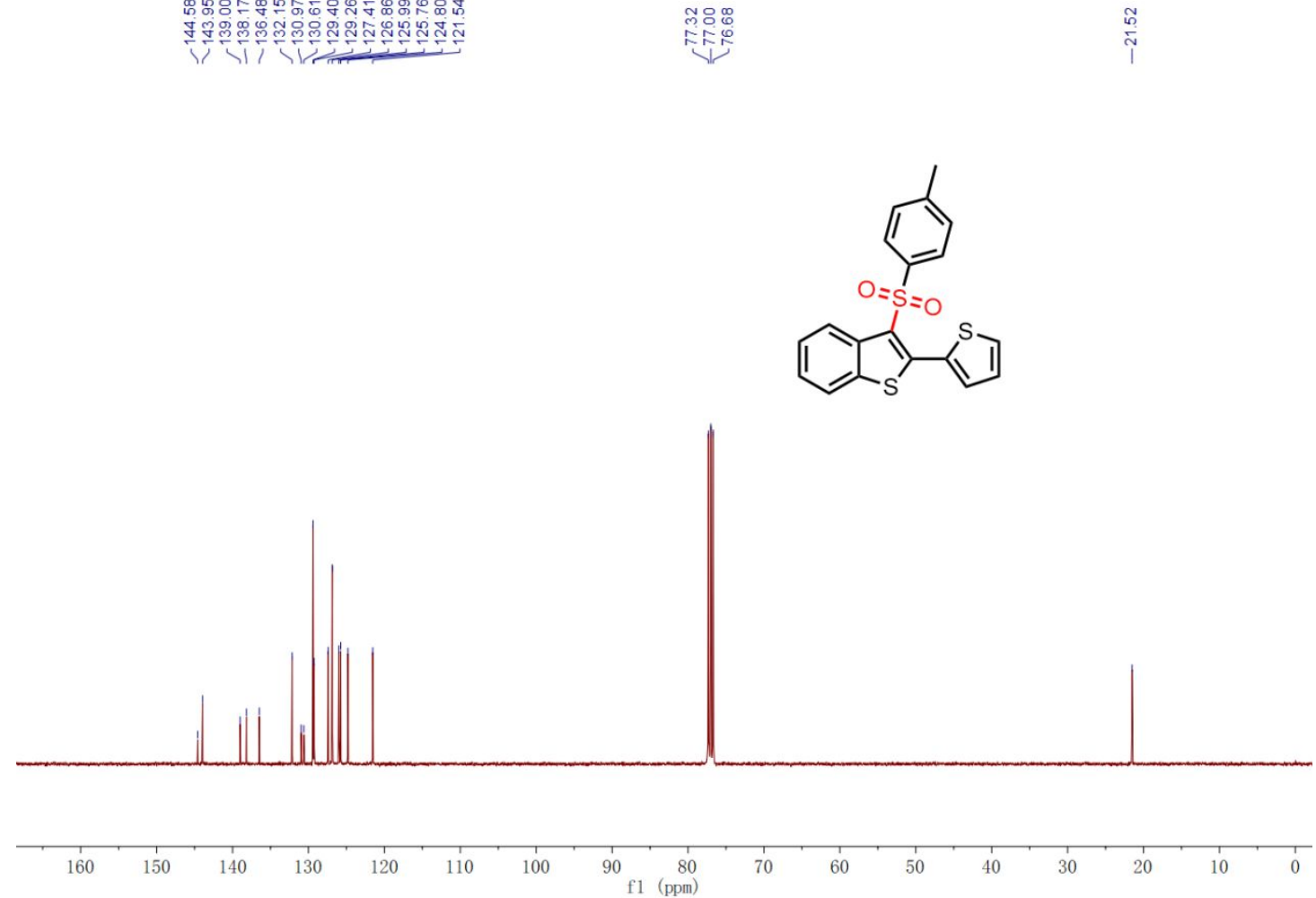

Figure S18. ${ }^{1} \mathrm{H}$ NMR of $3 \mathbf{r}\left(400 \mathrm{MHz}, \mathrm{CDCl}_{3}\right)$ and ${ }^{13} \mathrm{C}\left\{{ }^{1} \mathrm{H}\right\}$ NMR of $\mathbf{3 r}\left(100 \mathrm{MHz}, \mathrm{CDCl}_{3}\right)$ 
2-(Thiophen-3-yl)-3-tosylbenzo[b]thiophene (3s)

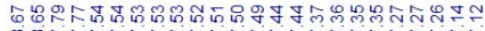
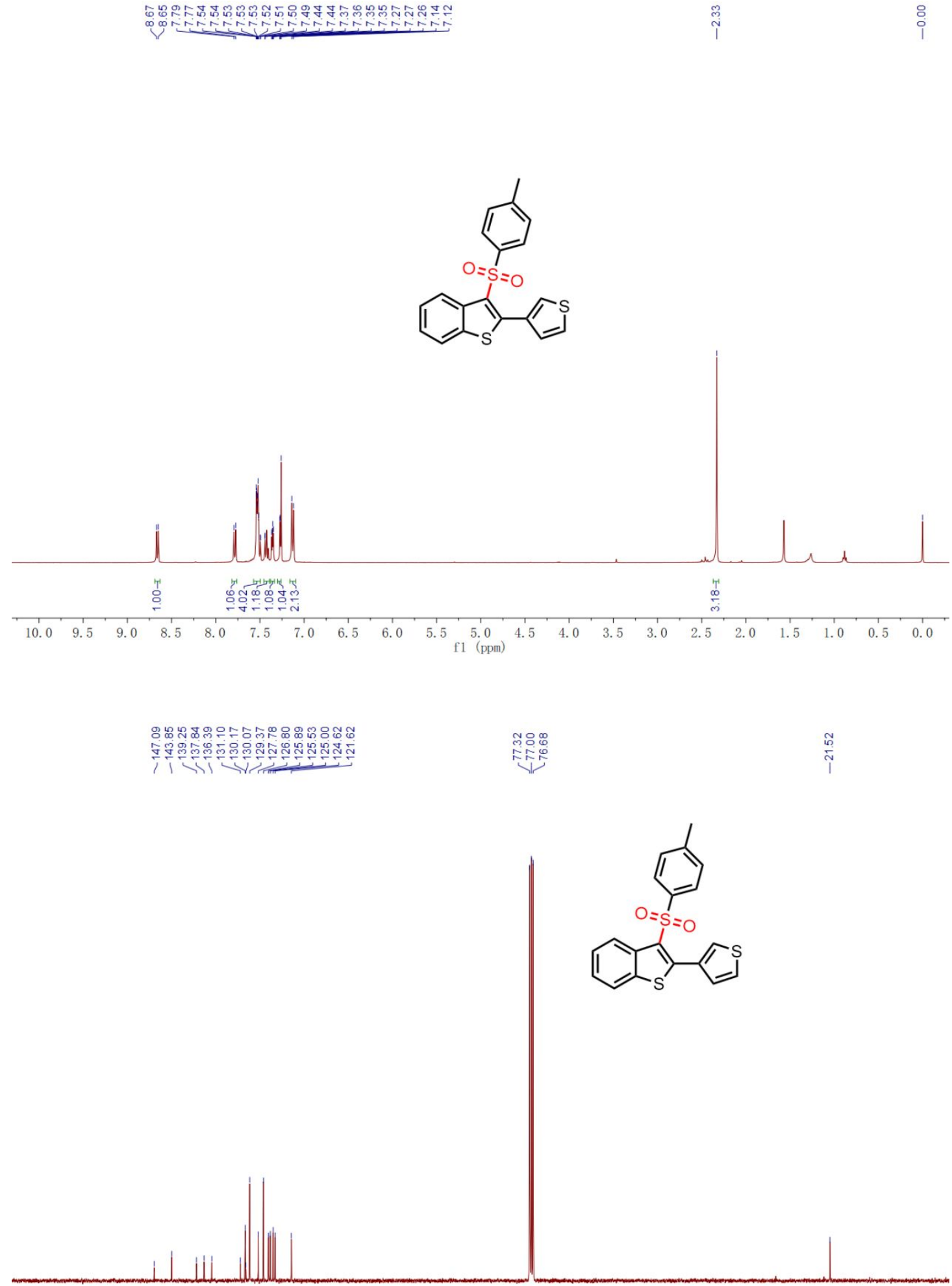

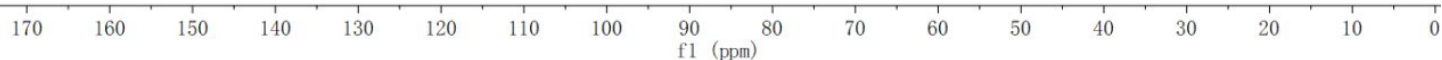

Figure S19. ${ }^{1} \mathrm{H}$ NMR of $3 \mathbf{s}\left(400 \mathrm{MHz}, \mathrm{CDCl}_{3}\right)$ and ${ }^{13} \mathrm{C}\left\{{ }^{1} \mathrm{H}\right\}$ NMR of $3 \mathbf{s}\left(100 \mathrm{MHz}, \mathrm{CDCl}_{3}\right)$ 
6-Methyl-2-phenyl-3-tosylbenzo[b]thiophene (3t)
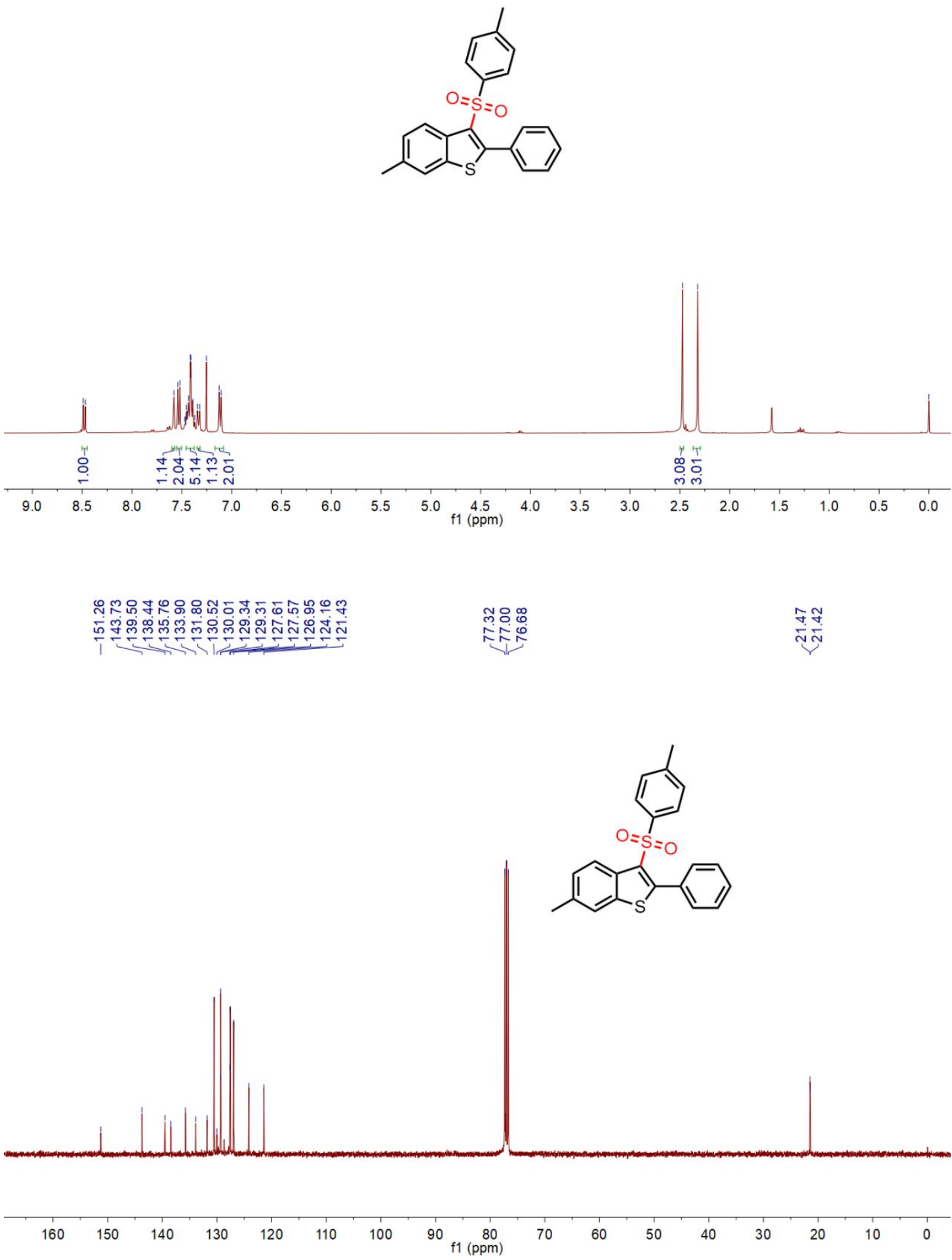

Figure S20. ${ }^{1} \mathrm{H}$ NMR of $3 \mathbf{t}\left(400 \mathrm{MHz}, \mathrm{CDCl}_{3}\right)$ and ${ }^{13} \mathrm{C}\left\{{ }^{1} \mathrm{H}\right\}$ NMR of $3 \mathbf{t}\left(100 \mathrm{MHz}, \mathrm{CDCl}_{3}\right)$ 
6-Fluoro-2-phenyl-3-tosylbenzo[b]thiophene (3u)

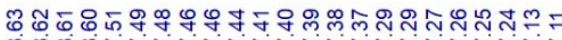

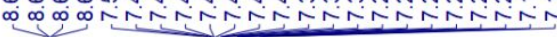

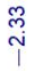
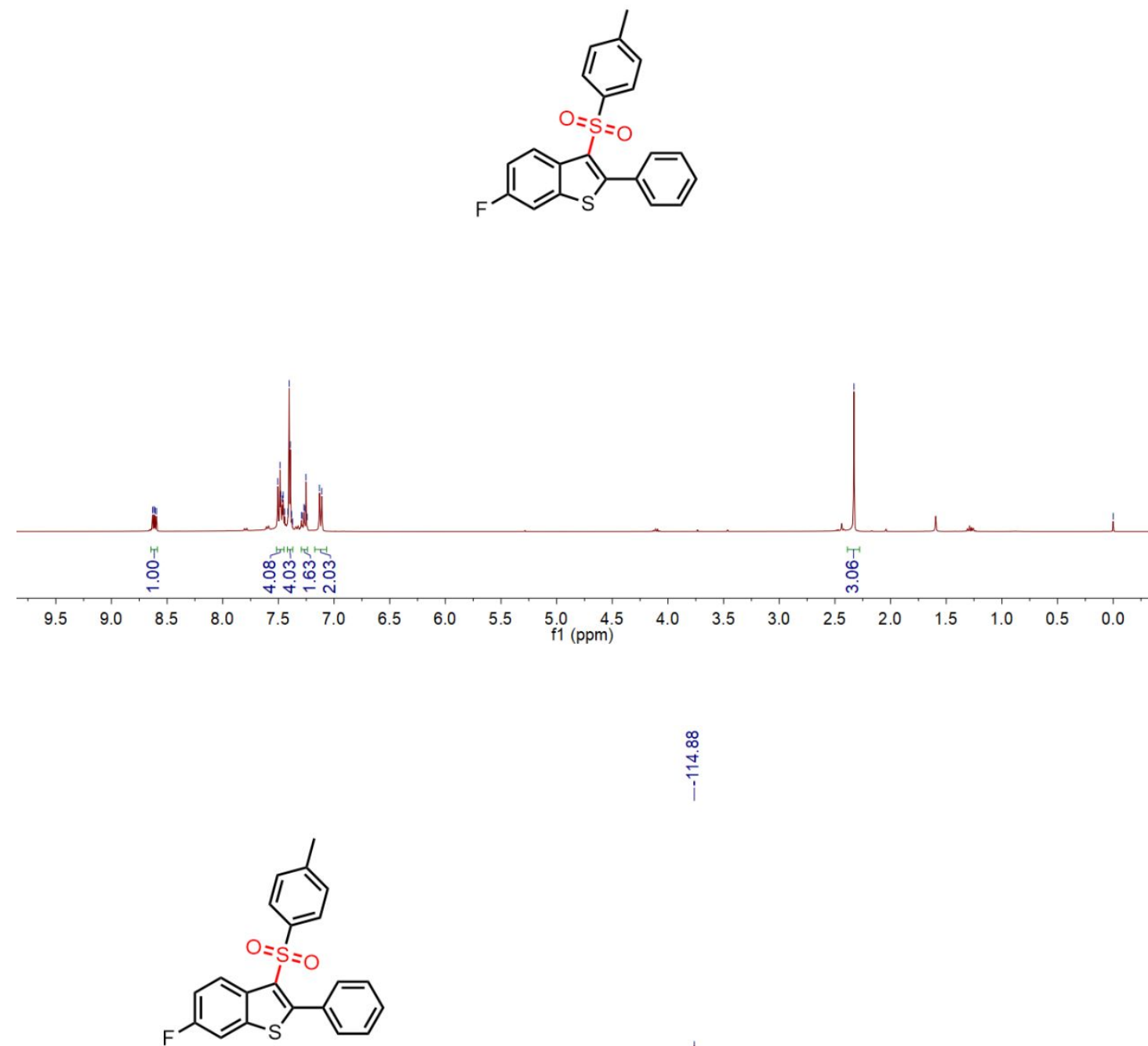

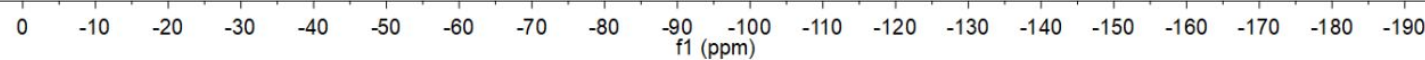



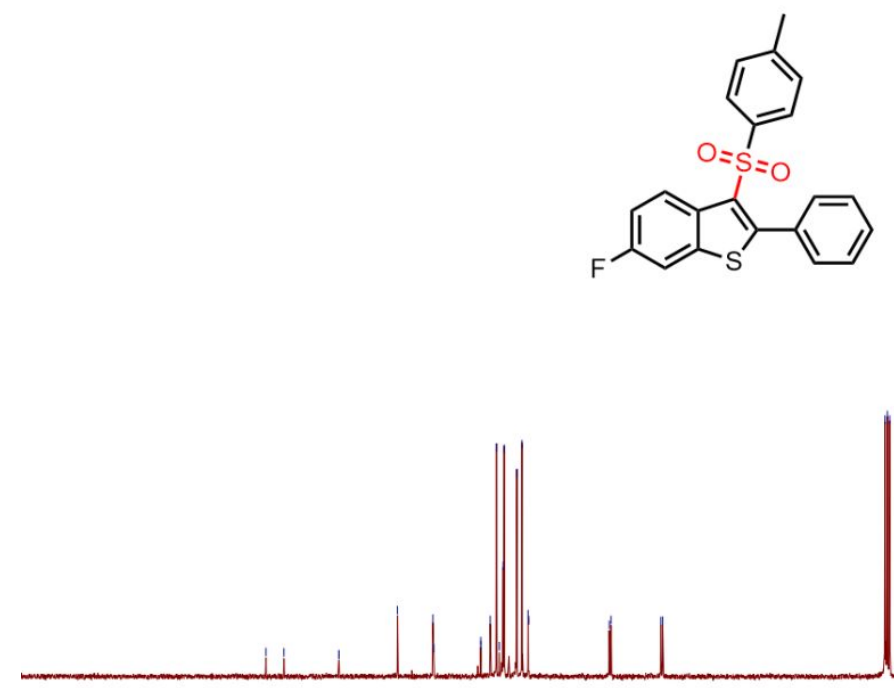

$\begin{array}{llllllllll}190 & 180 & 170 & 160 & 150 & 140 & 130 & 120 & 110 & \begin{array}{l}100 \\ \mathrm{f} 1(\mathrm{ppm})\end{array}\end{array}$

Figure S21. ${ }^{1} \mathrm{H}$ NMR of $3 \mathbf{u}\left(400 \mathrm{MHz}, \mathrm{CDCl}_{3}\right),{ }^{19} \mathrm{~F}$ NMR of $\mathbf{3 u}\left(376 \mathrm{MHz}, \mathrm{CDCl}_{3}\right)$ and ${ }^{13} \mathrm{C}\left\{{ }^{1} \mathrm{H}\right\}$ NMR of $3 \mathbf{u}\left(100 \mathrm{MHz}, \mathrm{CDCl}_{3}\right)$

6-Chloro-2-phenyl-3-tosylbenzo[b]thiophene (3v)

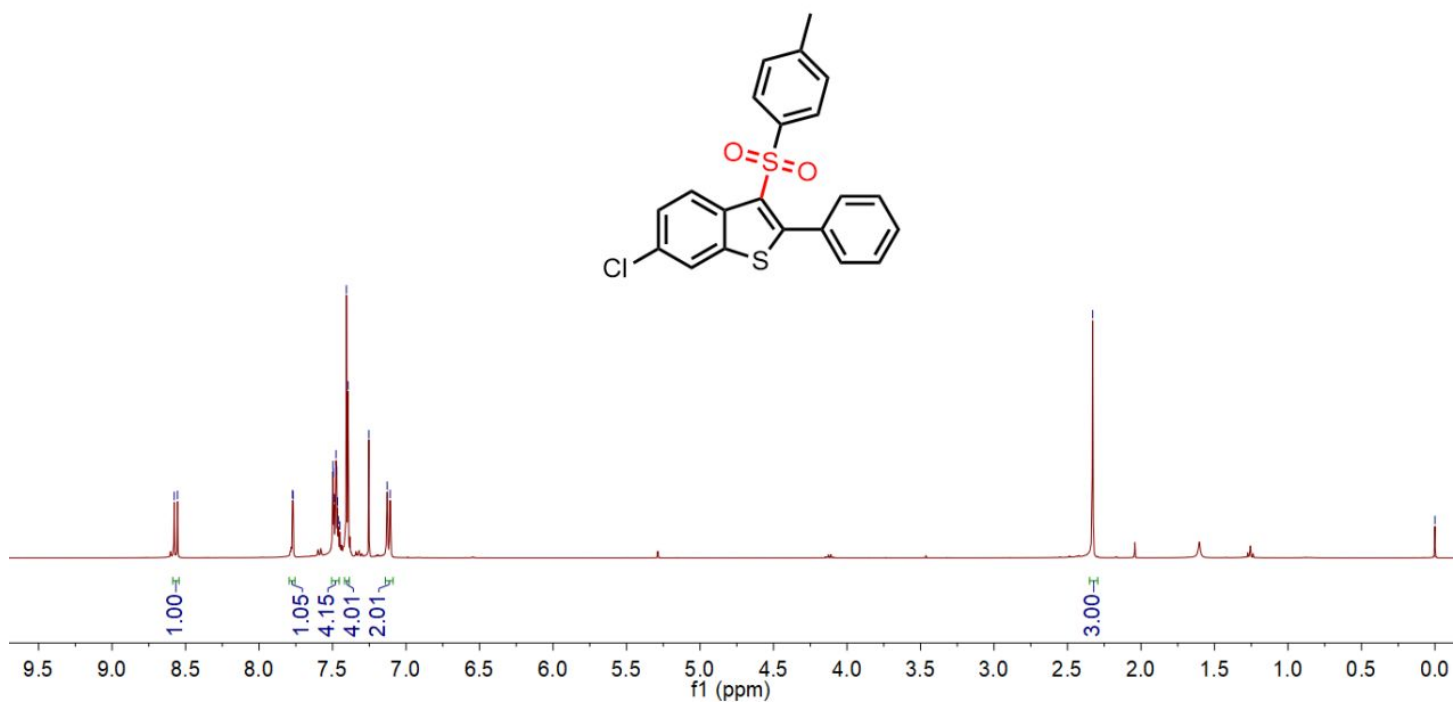



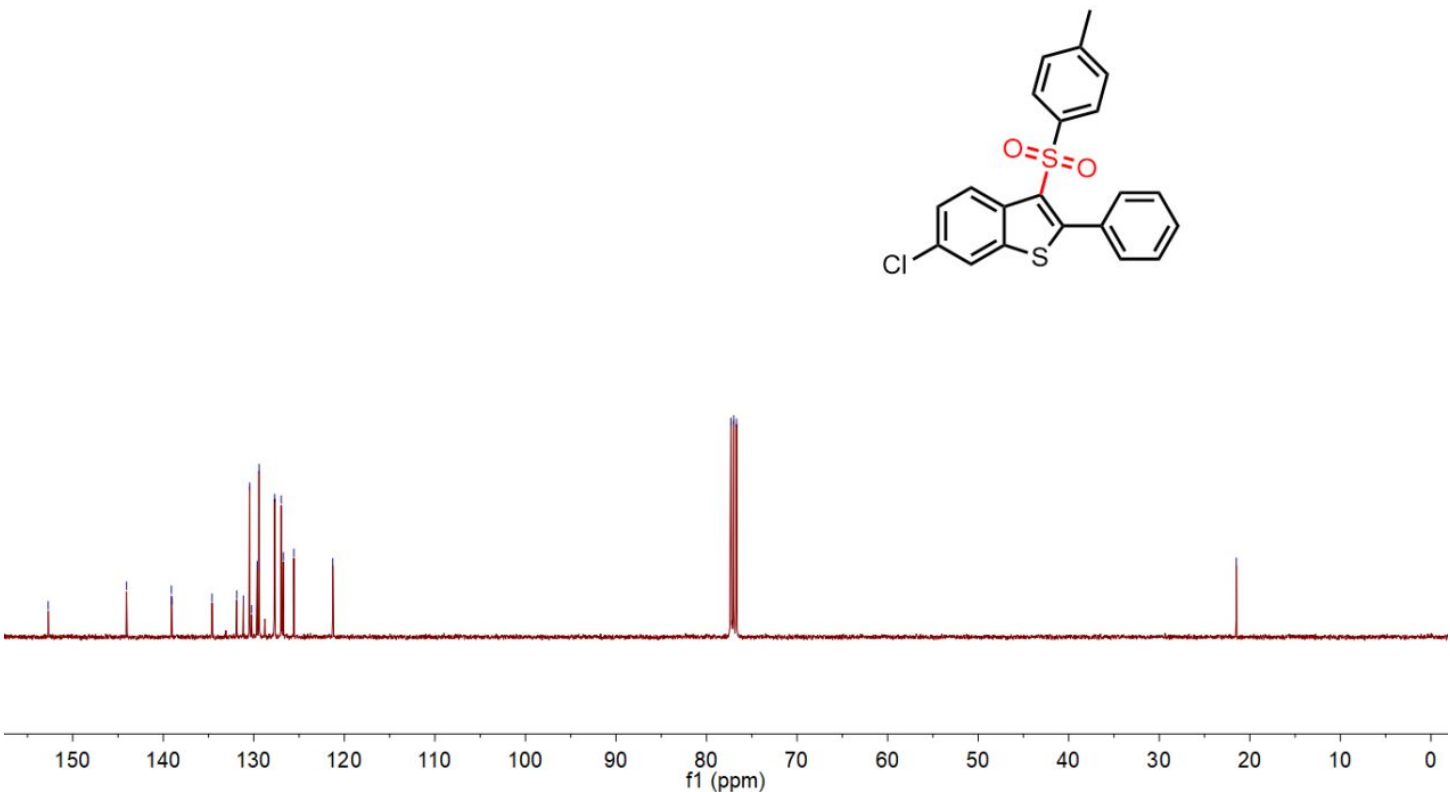

Figure S22. ${ }^{1} \mathrm{H}$ NMR of $\mathbf{3 v}\left(400 \mathrm{MHz}, \mathrm{CDCl}_{3}\right)$ and ${ }^{13} \mathrm{C}\left\{{ }^{1} \mathrm{H}\right\} \mathrm{NMR}$ of $\mathbf{3 v}\left(100 \mathrm{MHz}, \mathrm{CDCl}_{3}\right)$

6-Bromo-2-phenyl-3-tosylbenzo[b]thiophene (3w)
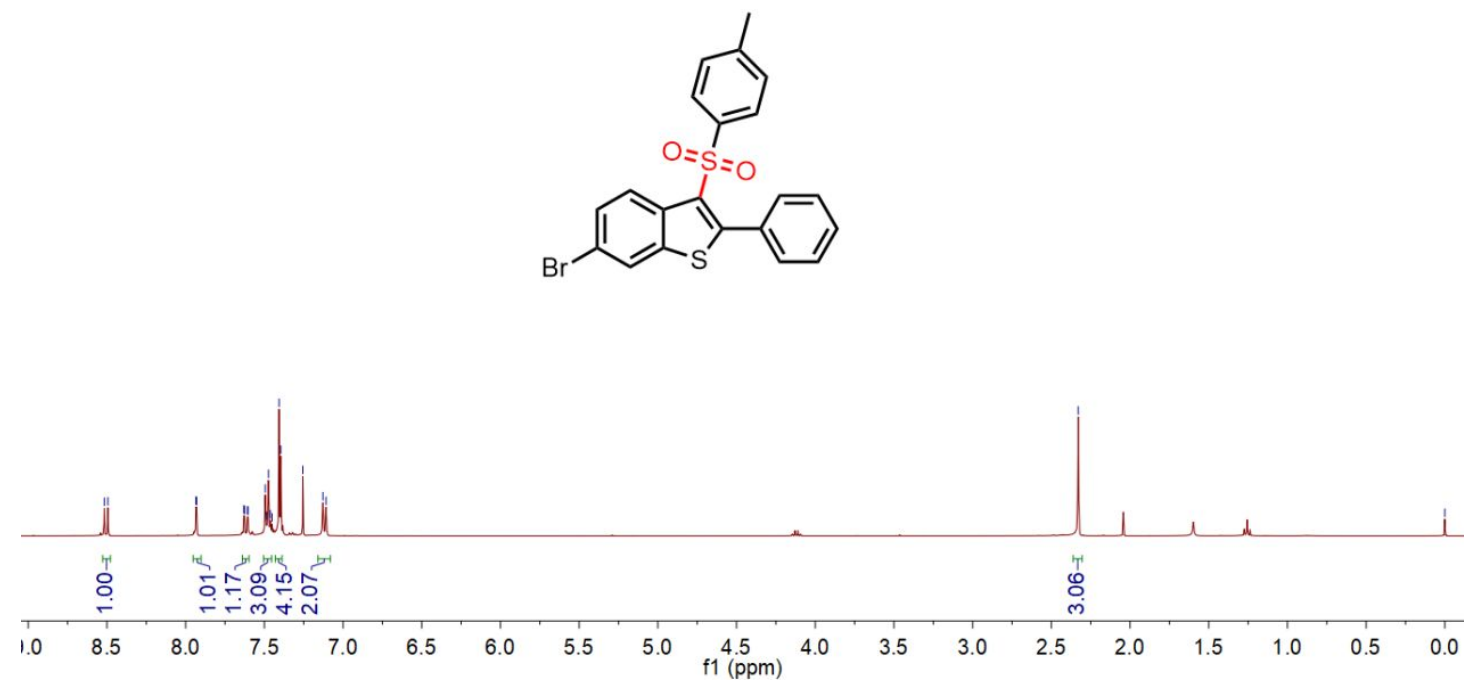


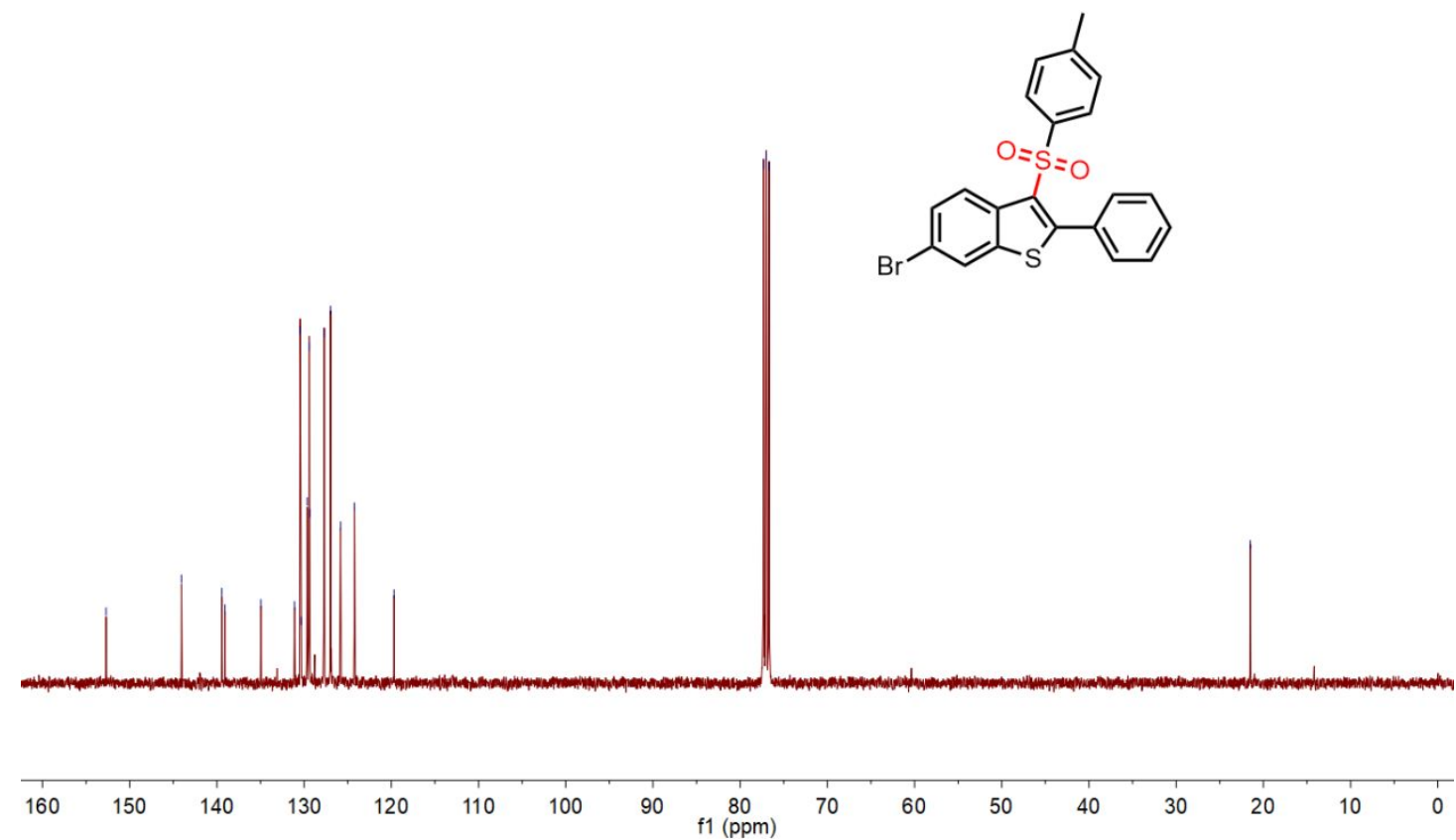

Figure S23. ${ }^{1} \mathrm{H}$ NMR of $\mathbf{3 w}\left(400 \mathrm{MHz}, \mathrm{CDCl}_{3}\right)$ and ${ }^{13} \mathrm{C}\left\{{ }^{1} \mathrm{H}\right\} \mathrm{NMR}$ of $\mathbf{3 w}\left(100 \mathrm{MHz}, \mathrm{CDCl}_{3}\right)$

2-Phenyl-3-(phenylsulfonyl) benzo[b]thiophene (3aa)

8.888888
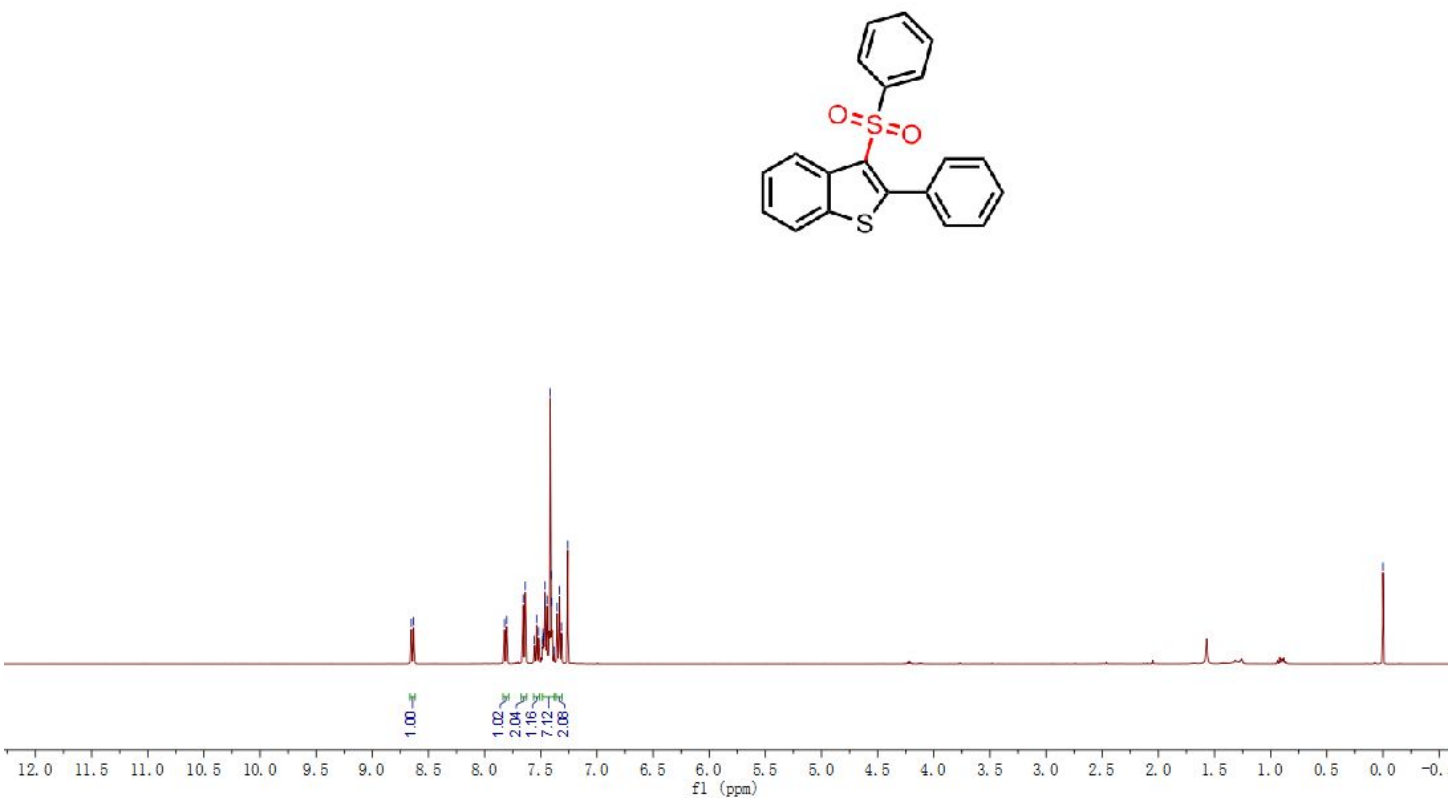


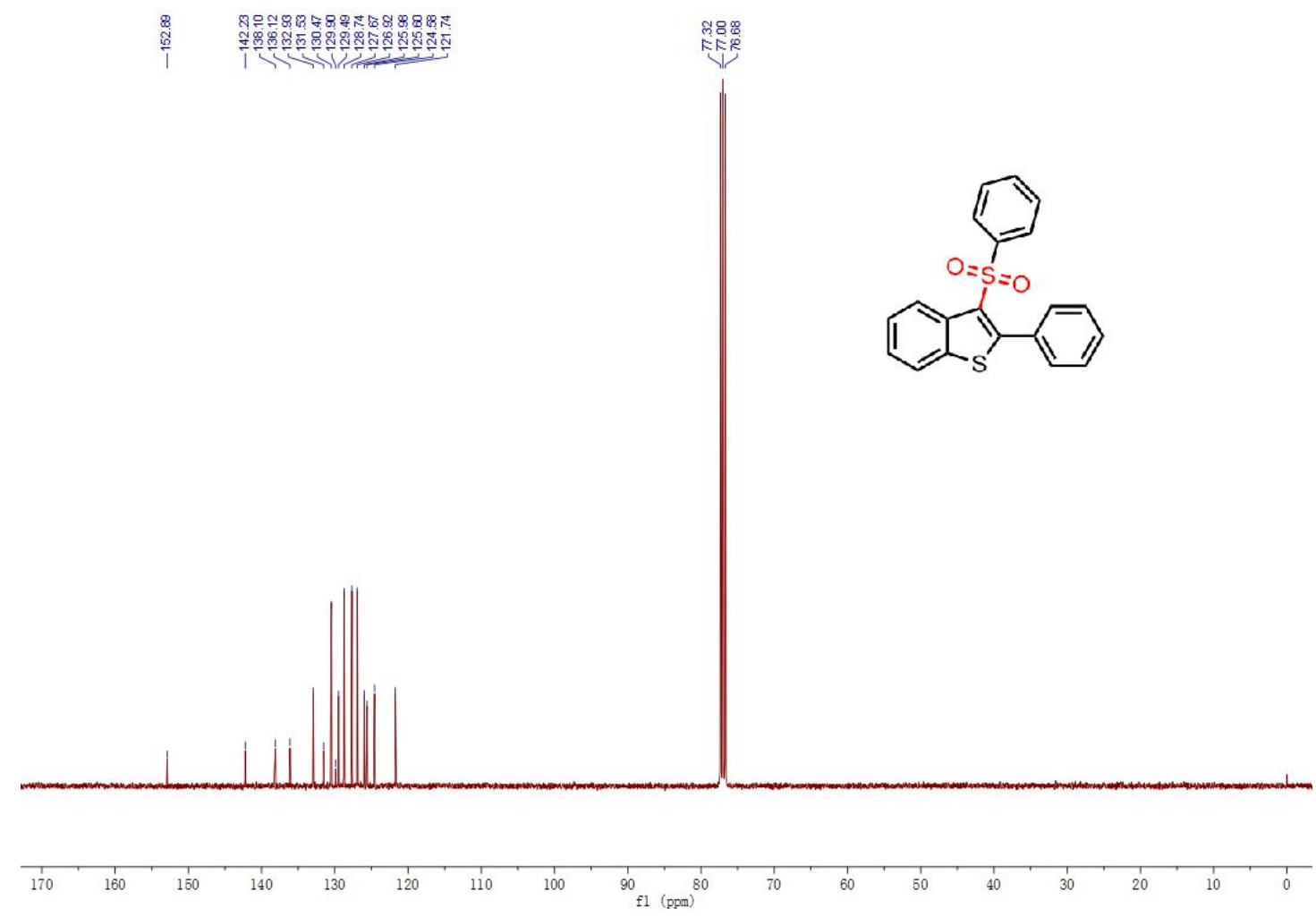

Figure S24. ${ }^{1} \mathrm{H}$ NMR of 3aa $\left(400 \mathrm{MHz}, \mathrm{CDCl}_{3}\right)$ and ${ }^{13} \mathrm{C}\left\{{ }^{1} \mathrm{H}\right\}$ NMR of $\mathbf{3 a a}\left(100 \mathrm{MHz}, \mathrm{CDCl}_{3}\right)$

2-Phenyl-3-(o-tolylsulfonyl) benzo[ $b]$ thiophene (3ab)

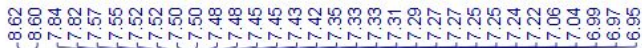
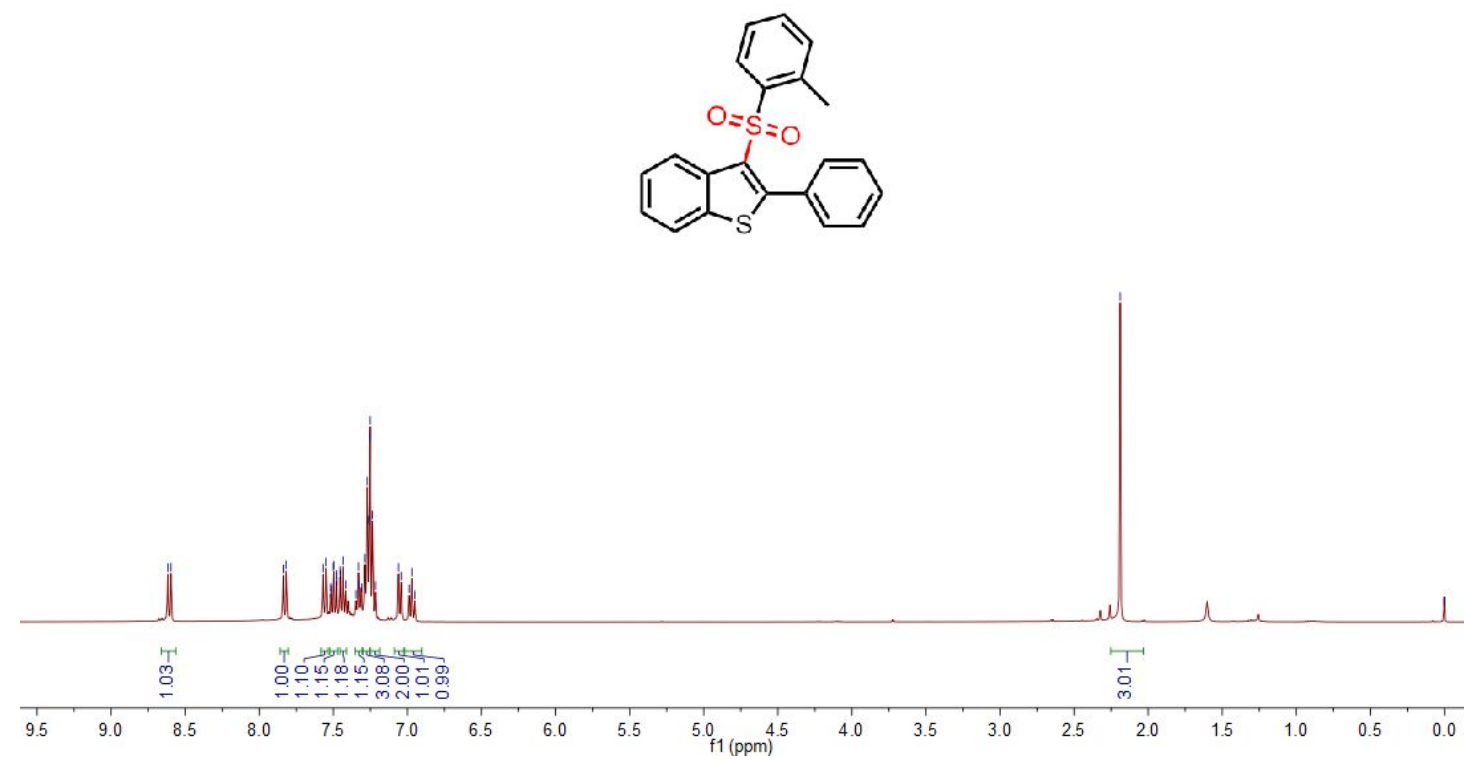

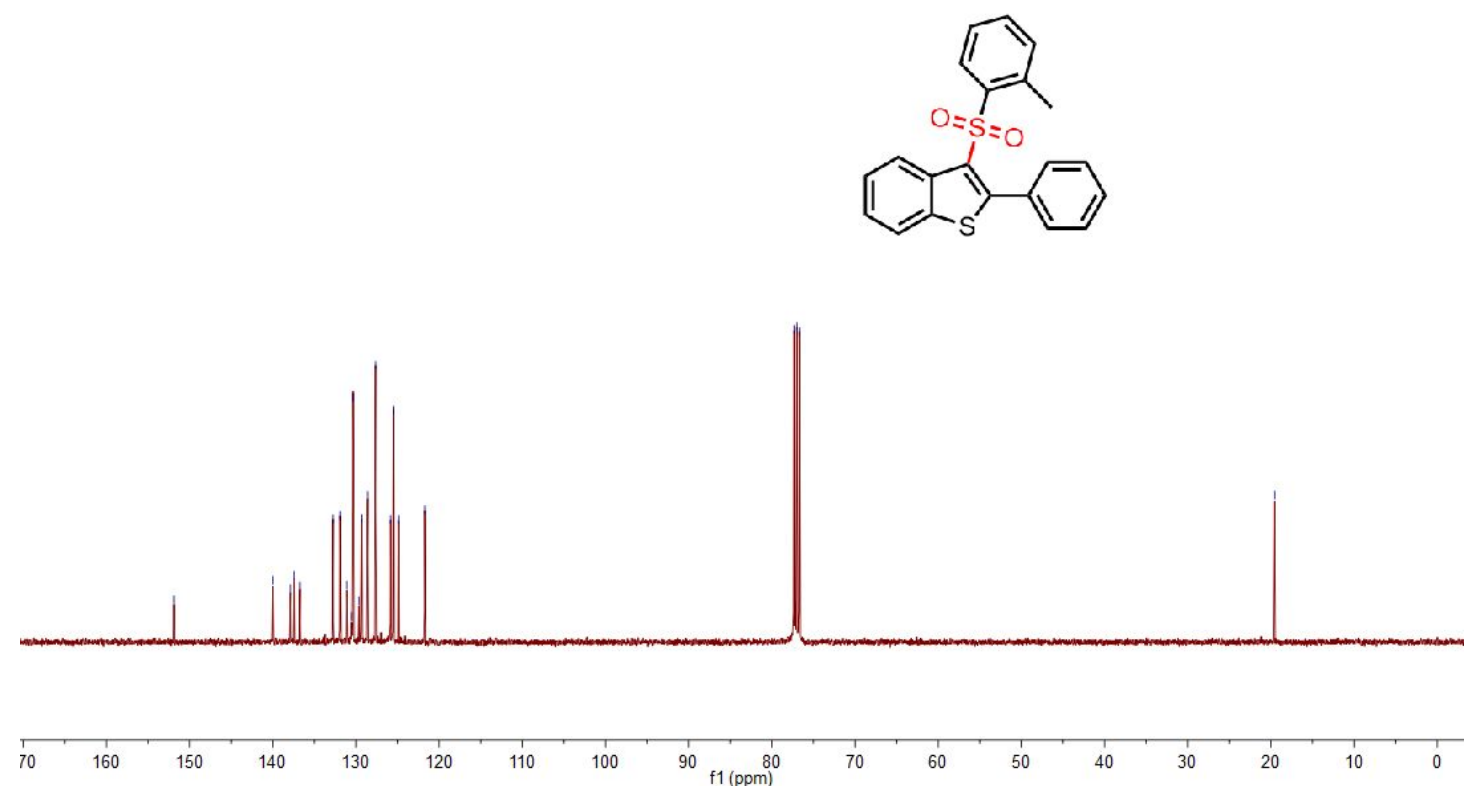

Figure S25. ${ }^{1} \mathrm{H}$ NMR of $\mathbf{3 a b}\left(400 \mathrm{MHz}, \mathrm{CDCl}_{3}\right)$ and ${ }^{13} \mathrm{C}\left\{{ }^{1} \mathrm{H}\right\}$ NMR of $\mathbf{3 a b}\left(100 \mathrm{MHz}, \mathrm{CDCl}_{3}\right)$

3-((2-Chlorophenyl) sulfonyl)-2-phenylbenzo[b]thiophene (3ac)

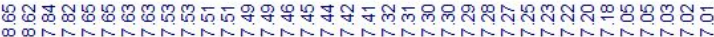

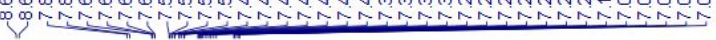
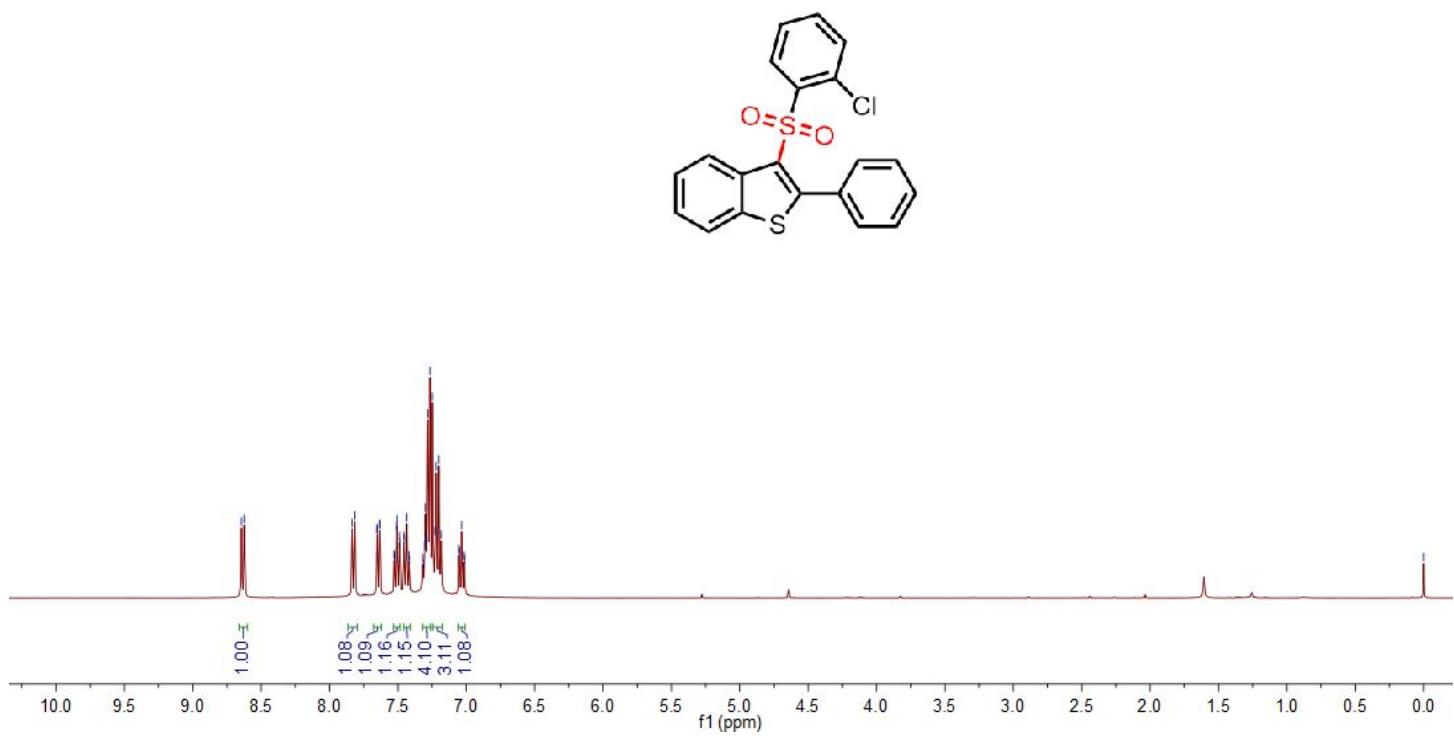

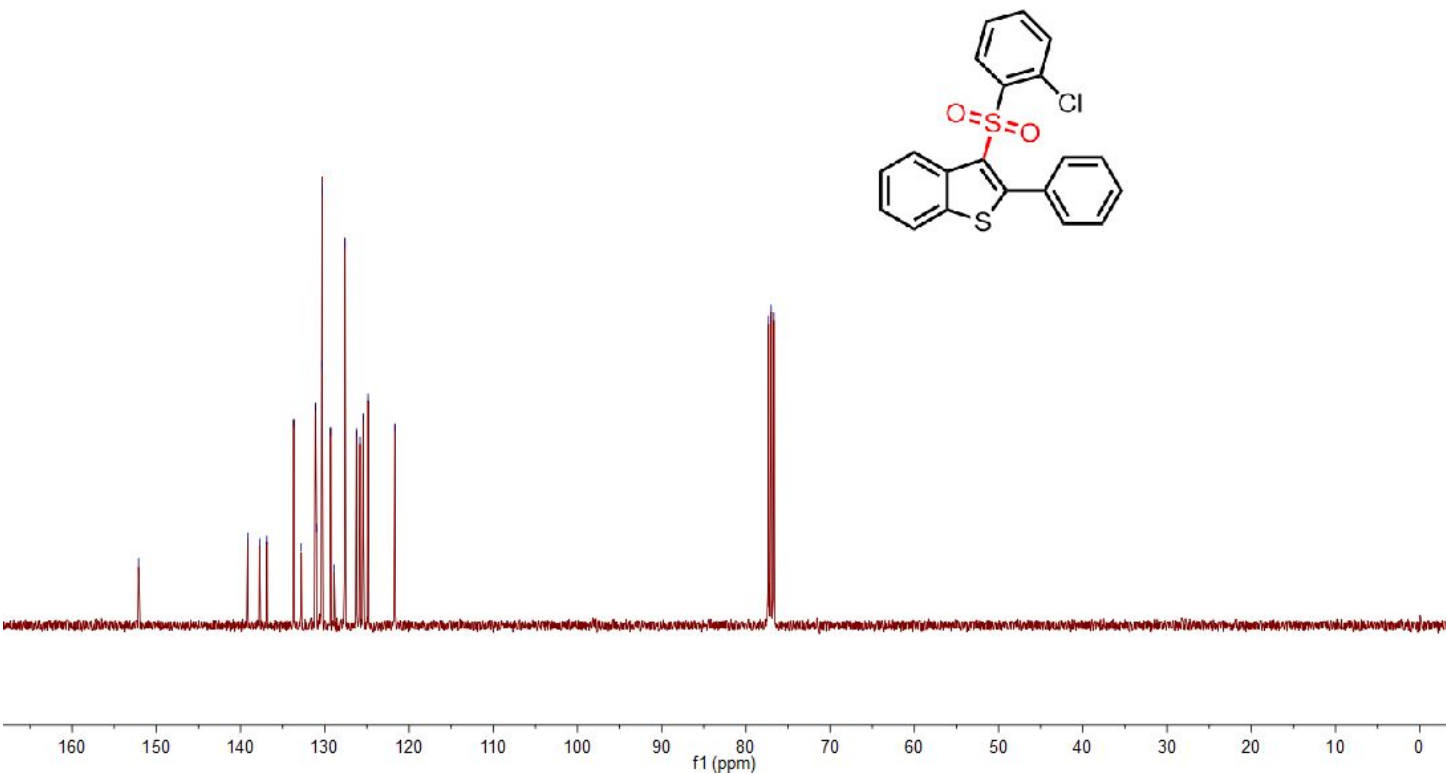

Figure S26. ${ }^{1} \mathrm{H}$ NMR of $\mathbf{3 a c}\left(400 \mathrm{MHz}, \mathrm{CDCl}_{3}\right)$ and ${ }^{13} \mathrm{C}\left\{{ }^{1} \mathrm{H}\right\}$ NMR of $\mathbf{3 a c}\left(100 \mathrm{MHz}, \mathrm{CDCl}_{3}\right)$

3-((2-Bromophenyl) sulfonyl)-2-phenylbenzo[b]thiophene (3ad)

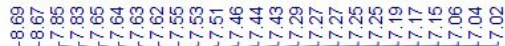
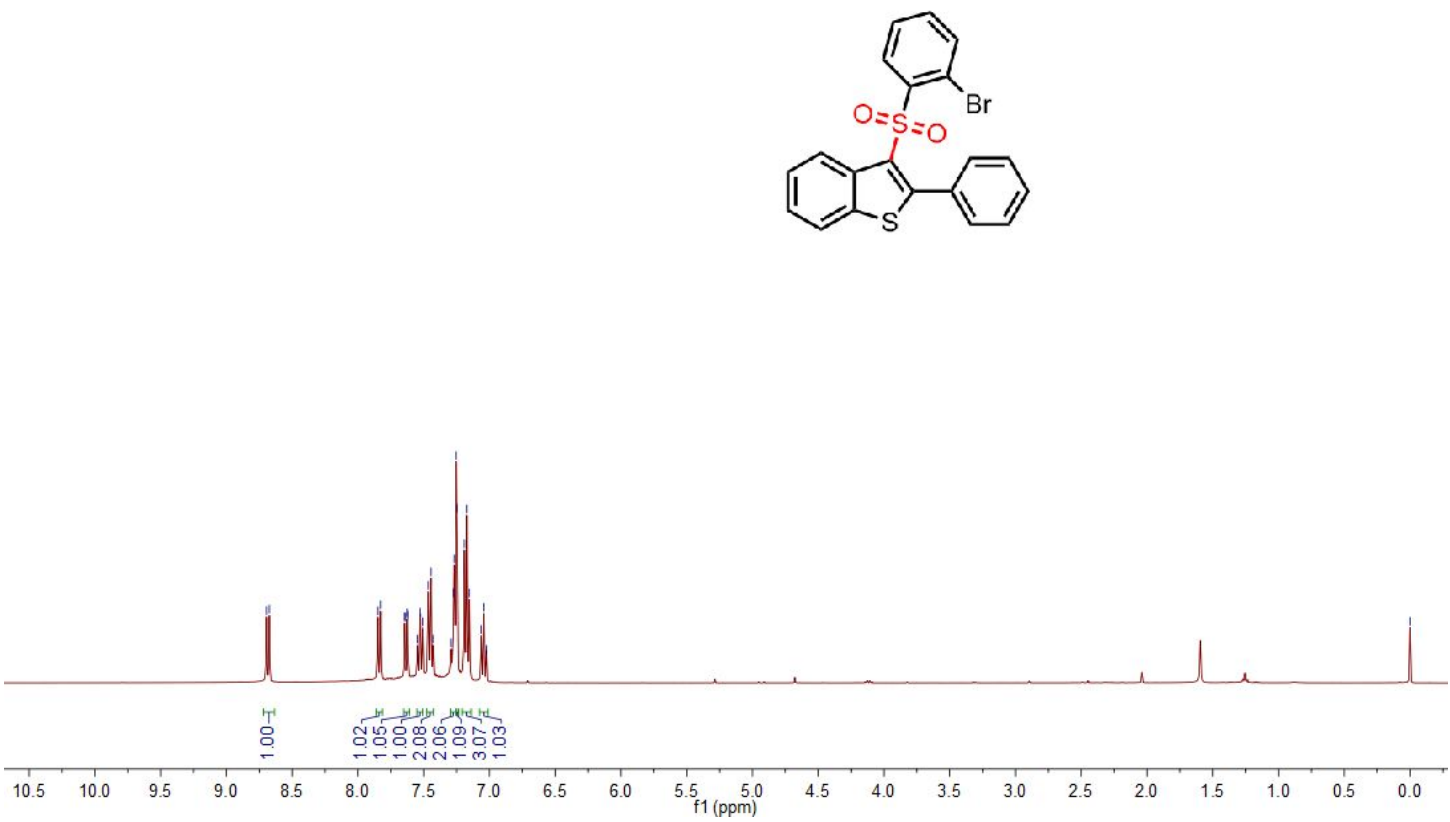

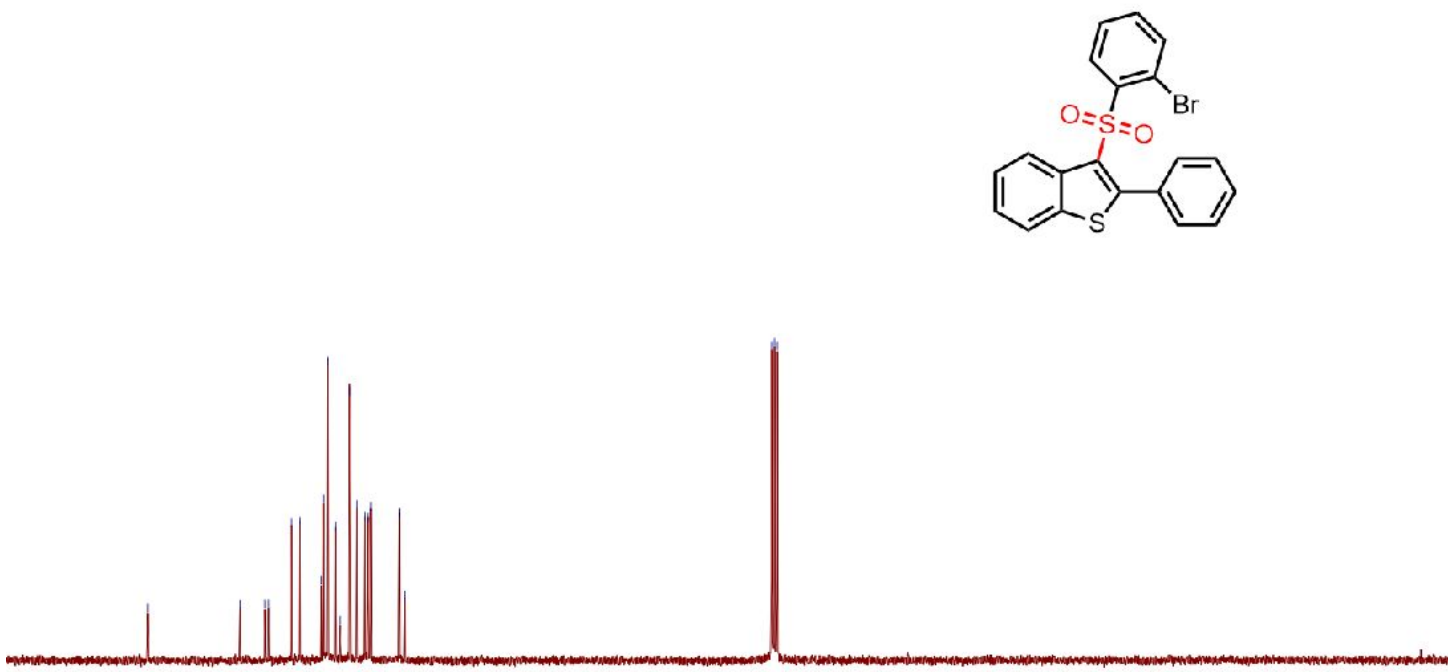

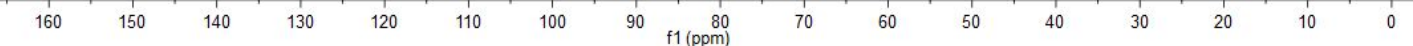

Figure S27. ${ }^{1} \mathrm{H}$ NMR of $\mathbf{3 a d}\left(400 \mathrm{MHz}, \mathrm{CDCl}_{3}\right)$ and ${ }^{13} \mathrm{C}\left\{{ }^{1} \mathrm{H}\right\}$ NMR of $\mathbf{3 a d}\left(100 \mathrm{MHz}, \mathrm{CDCl}_{3}\right)$

3-((4-Methoxyphenyl) sulfonyl)-2-phenylbenzo[b]thiophene (3ae)
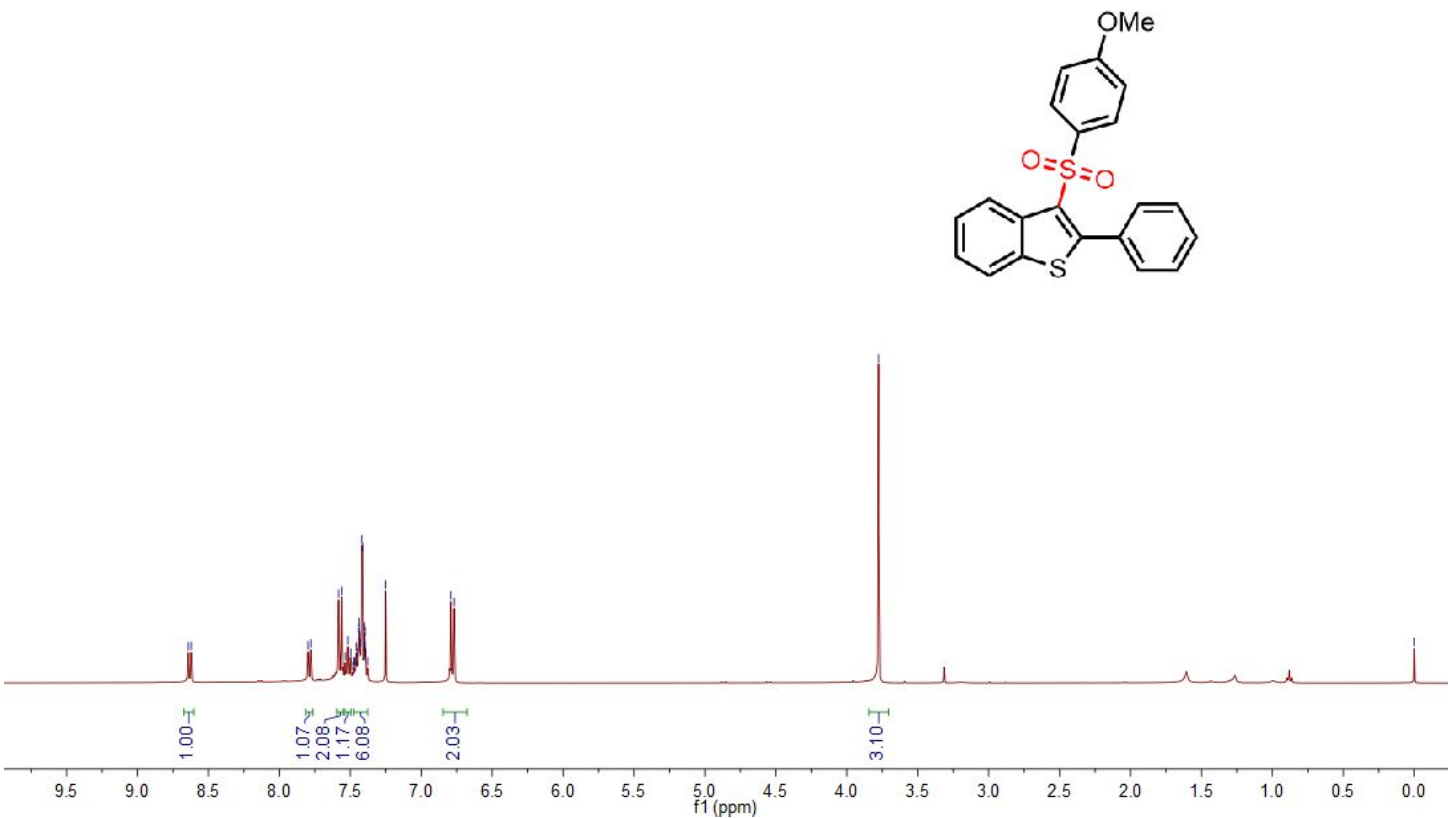

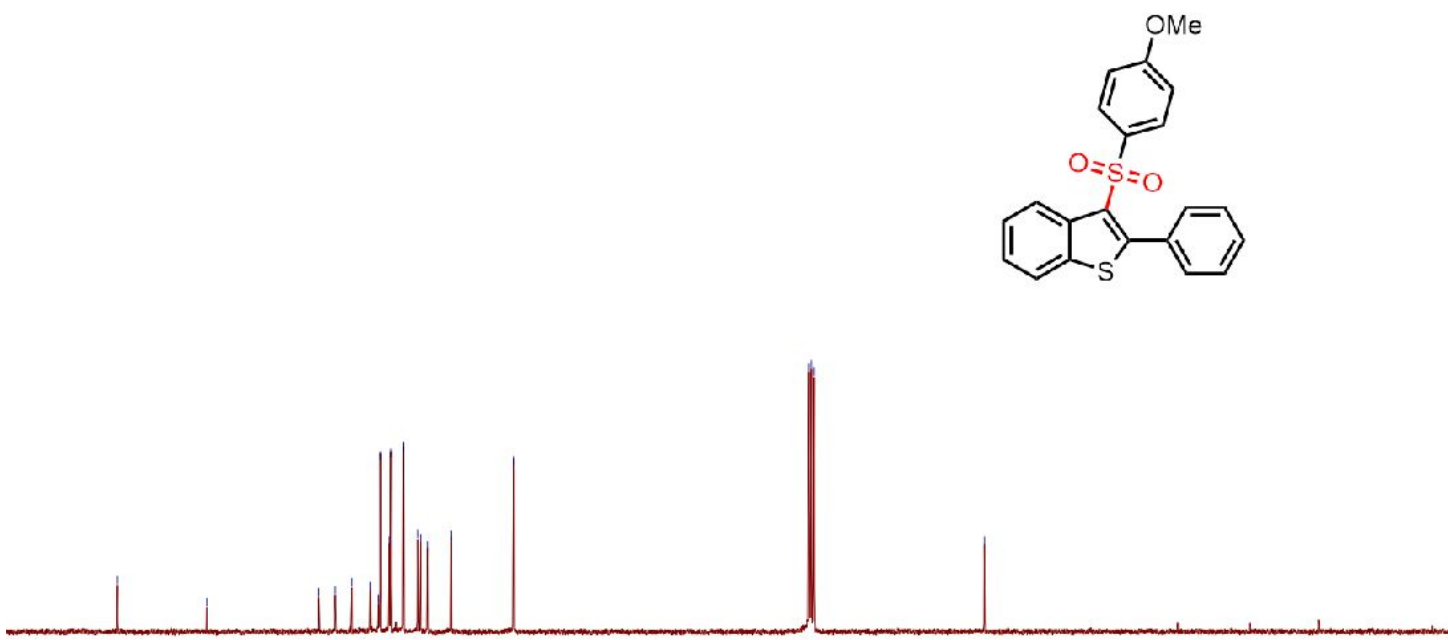

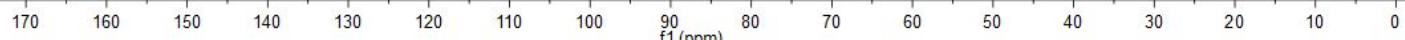

Figure S28. ${ }^{1} \mathrm{H}$ NMR of 3ae $\left(400 \mathrm{MHz}, \mathrm{CDCl}_{3}\right)$ and ${ }^{13} \mathrm{C}\left\{{ }^{1} \mathrm{H}\right\}$ NMR of $3 a e\left(100 \mathrm{MHz}, \mathrm{CDCl}_{3}\right)$

3-((4-(Tert-butyl) phenyl) sulfonyl)-2-phenylbenzo[b]thiophene (3af)

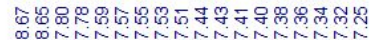

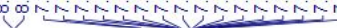
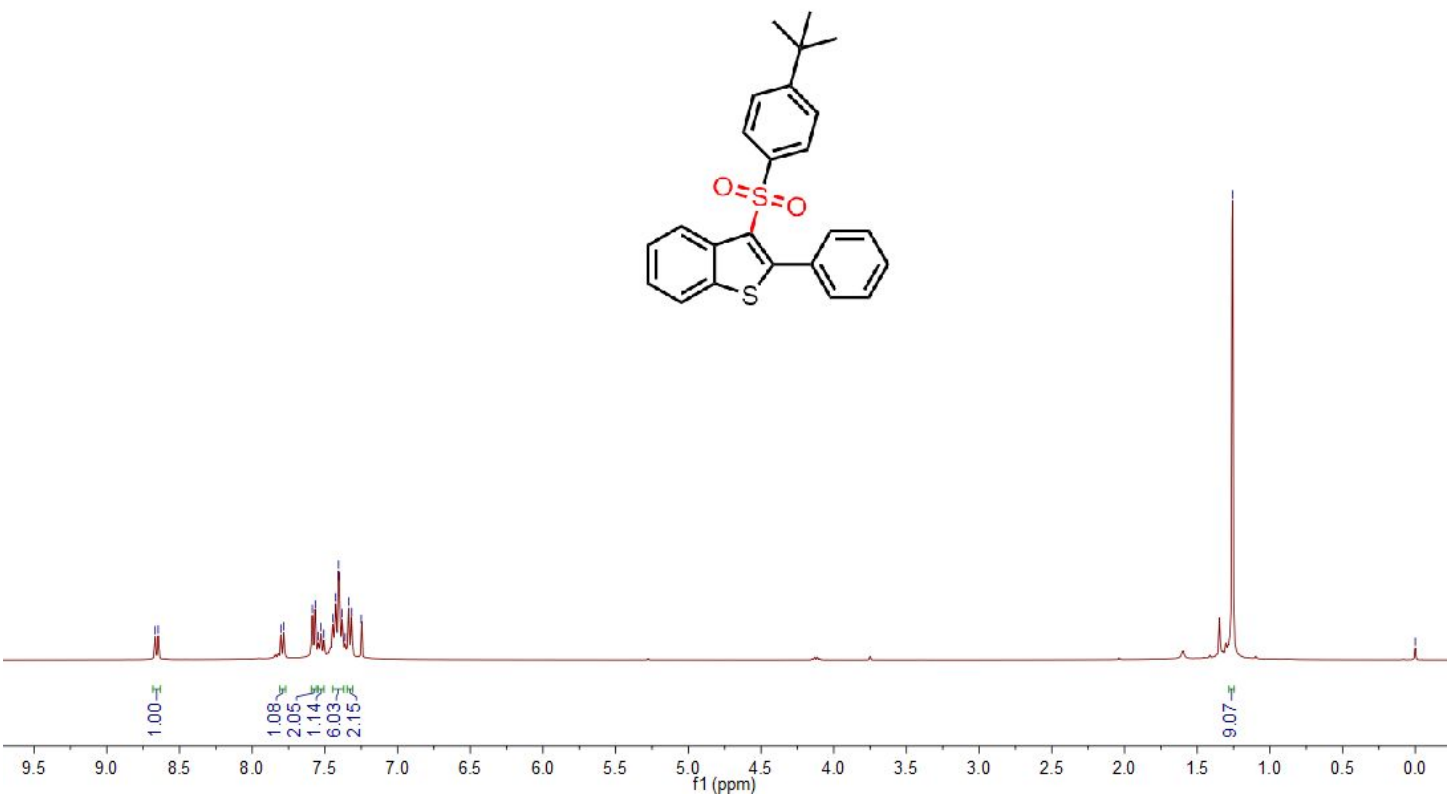

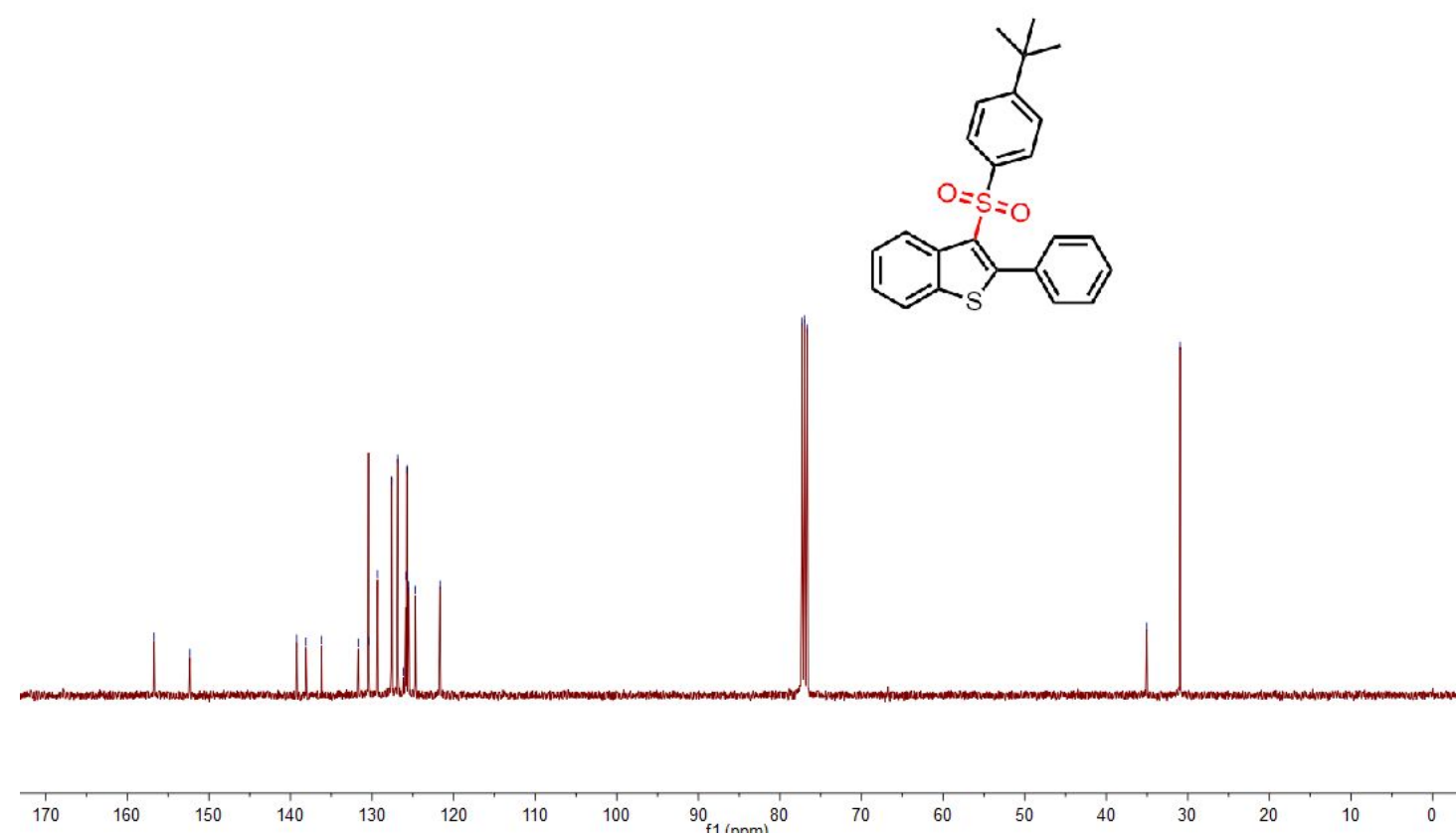

Figure S29. ${ }^{1} \mathrm{H}$ NMR of $\mathbf{3 a f}\left(400 \mathrm{MHz}, \mathrm{CDCl}_{3}\right)$ and ${ }^{13} \mathrm{C}\left\{{ }^{1} \mathrm{H}\right\}$ NMR of $\mathbf{3 a f}\left(100 \mathrm{MHz}, \mathrm{CDCl}_{3}\right)$

3-((4-Fluorophenyl) sulfonyl)-2-phenylbenzo[b]thiophene (3ag)

8885888968896518 .
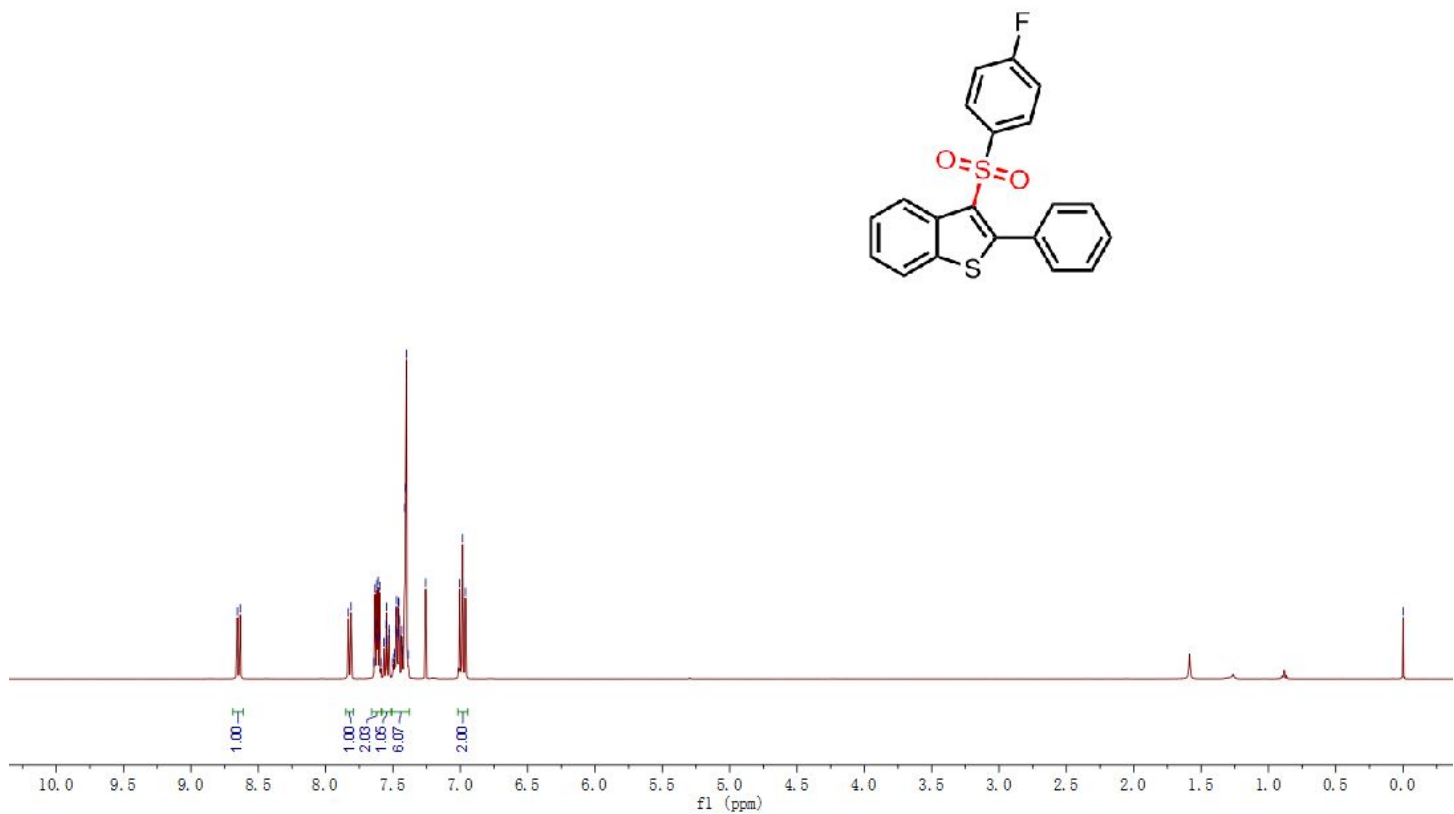

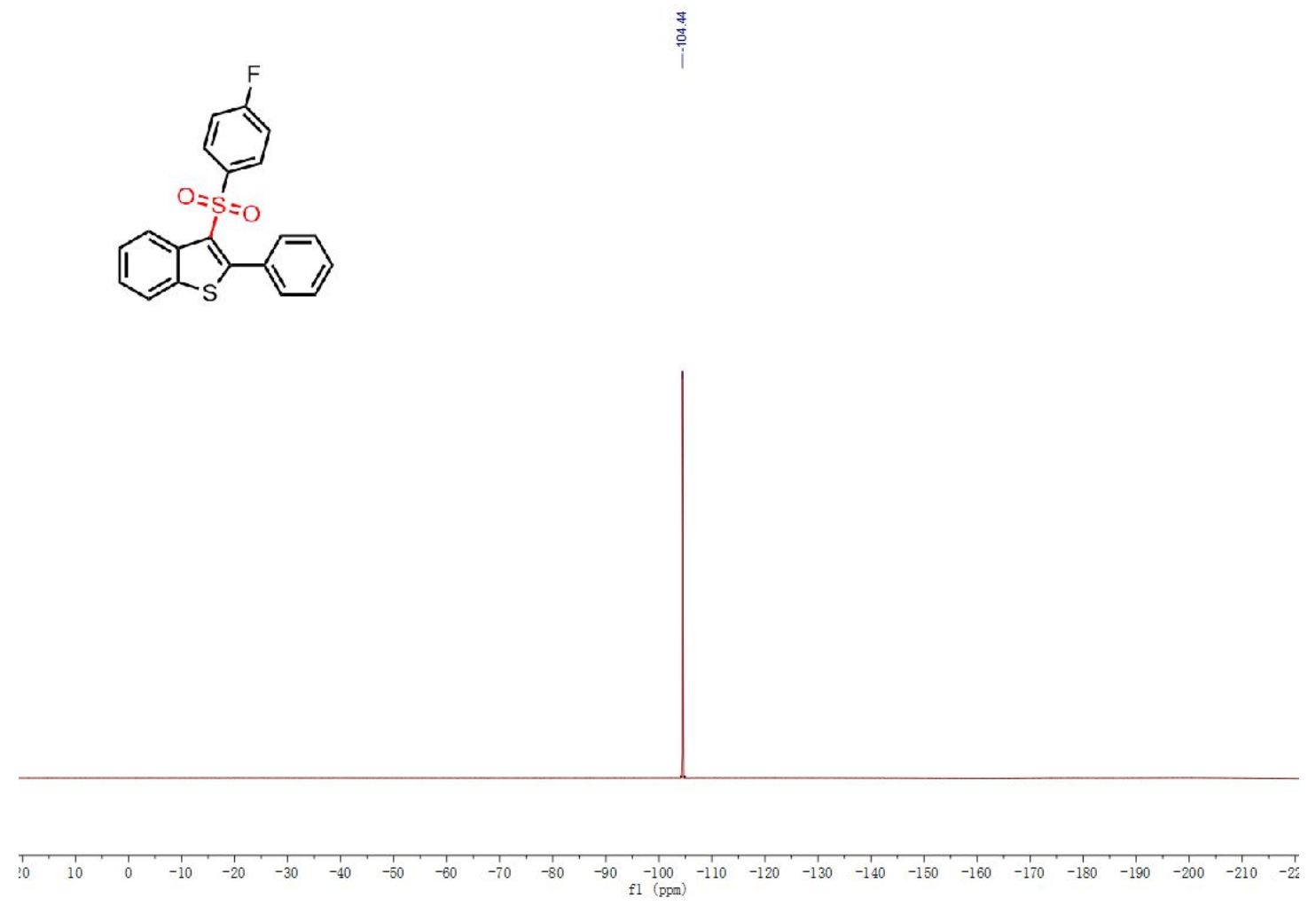

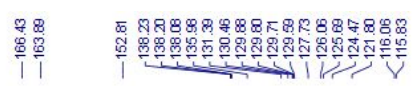

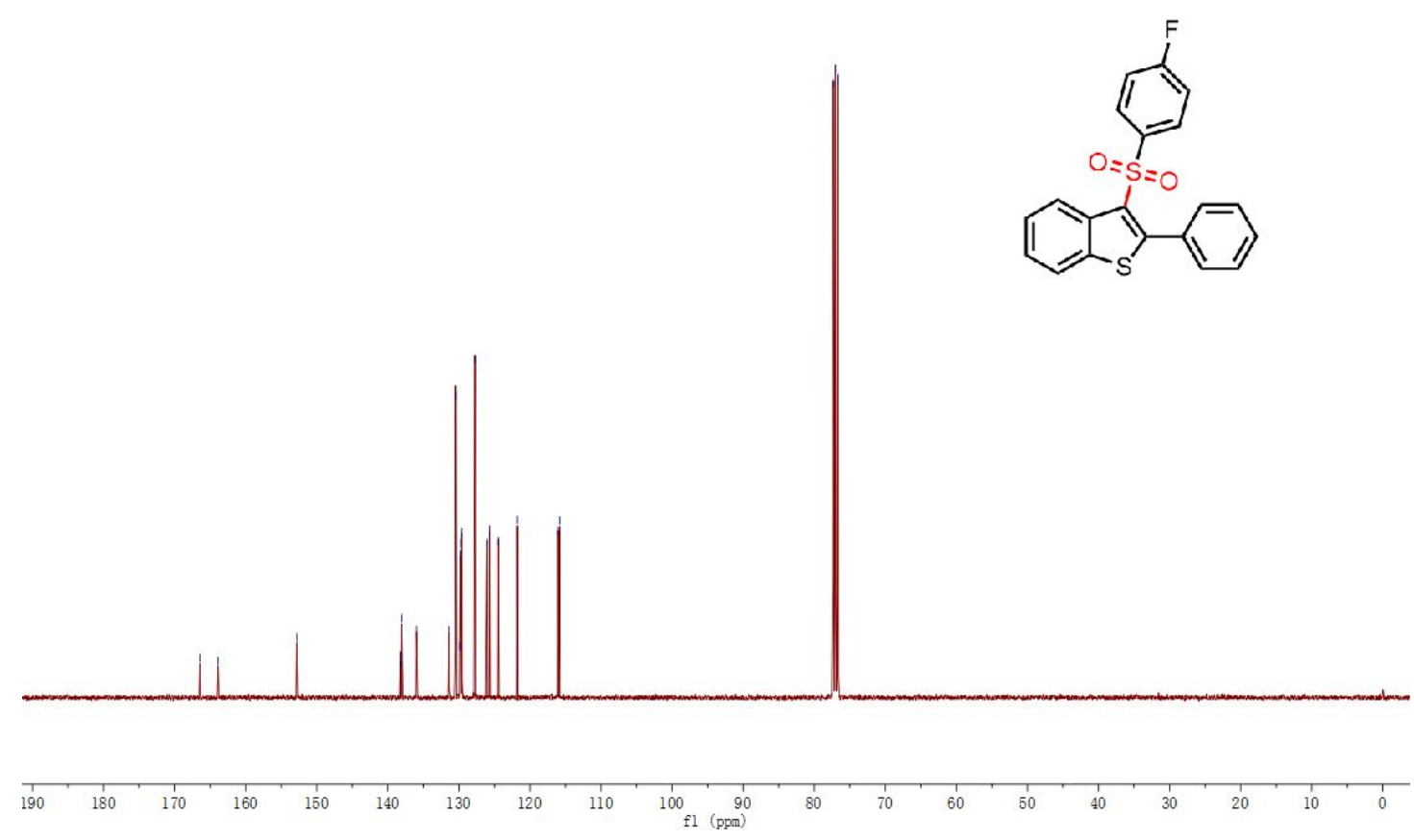

Figure S30. ${ }^{1} \mathrm{H}$ NMR of $\mathbf{3 a g}\left(400 \mathrm{MHz}, \mathrm{CDCl}_{3}\right),{ }^{19} \mathrm{~F}$ NMR of $\mathbf{3 a g}\left(376 \mathrm{MHz}, \mathrm{CDCl}_{3}\right)$ and ${ }^{13} \mathrm{C}\left\{{ }^{1} \mathrm{H}\right\}$ NMR of $\mathbf{3 a g}\left(100 \mathrm{MHz}, \mathrm{CDCl}_{3}\right)$ 
3-((4-Chlorophenyl) sulfonyl)-2-phenylbenzo[b]thiophene (3ah)

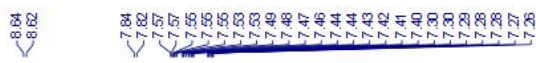
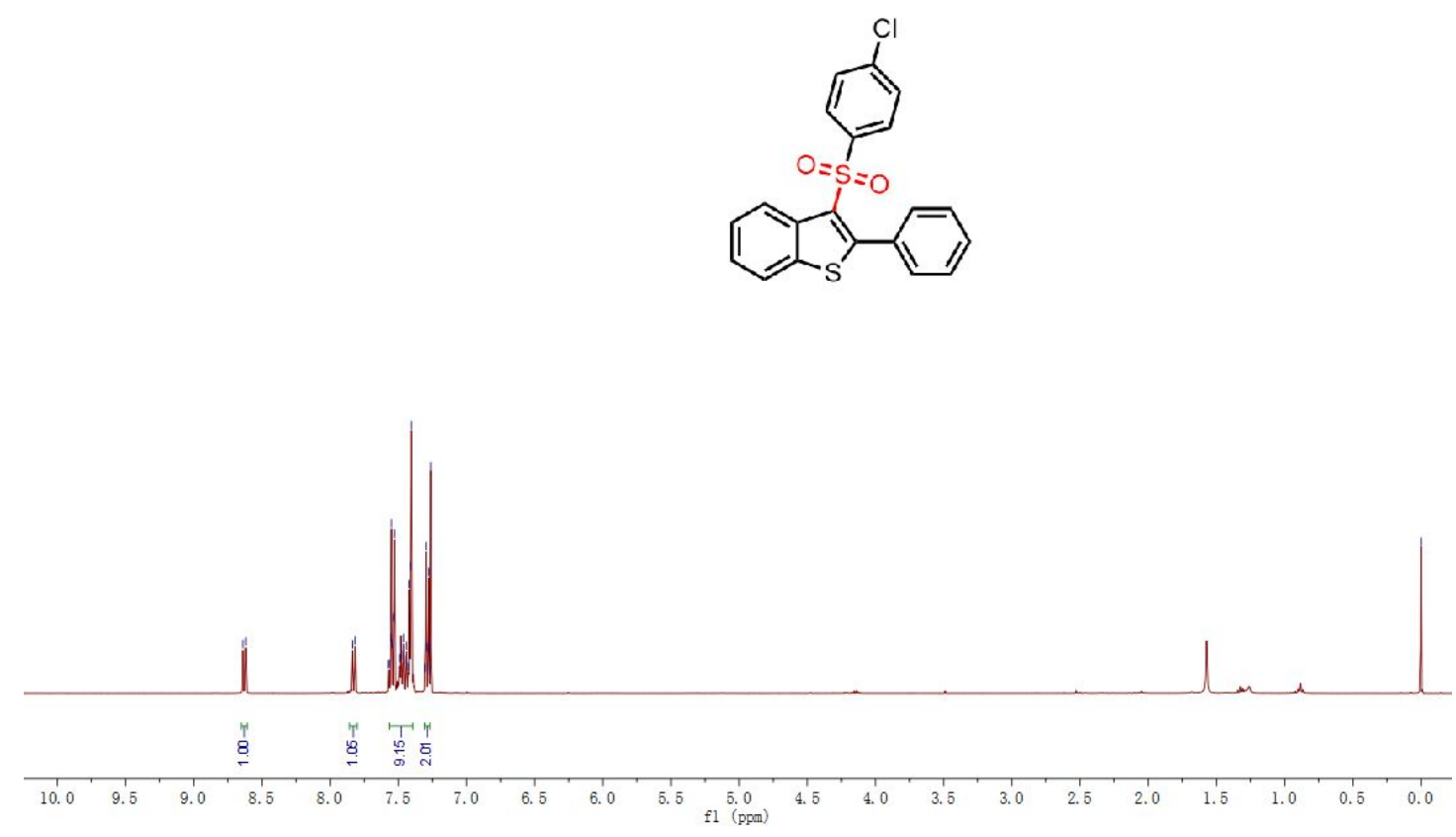

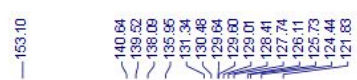
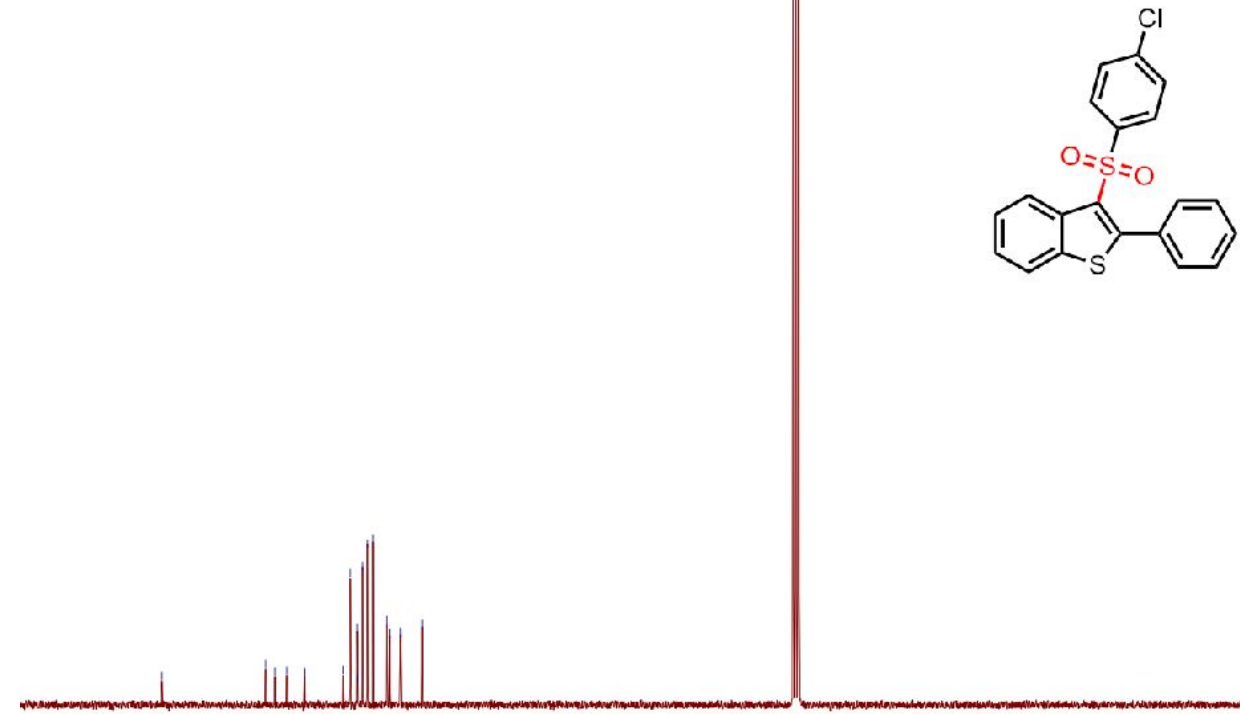

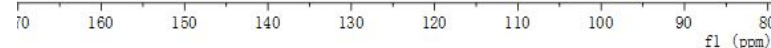

Figure S31. ${ }^{1} \mathrm{H}$ NMR of $\mathbf{3 a h}\left(400 \mathrm{MHz}, \mathrm{CDCl}_{3}\right)$ and ${ }^{13} \mathrm{C}\left\{{ }^{1} \mathrm{H}\right\} \mathrm{NMR}$ of $\mathbf{3 a h}\left(100 \mathrm{MHz}, \mathrm{CDCl}_{3}\right)$ 
3-((4-Bromophenyl) sulfonyl)-2-phenylbenzo[b]thiophene (3ai)
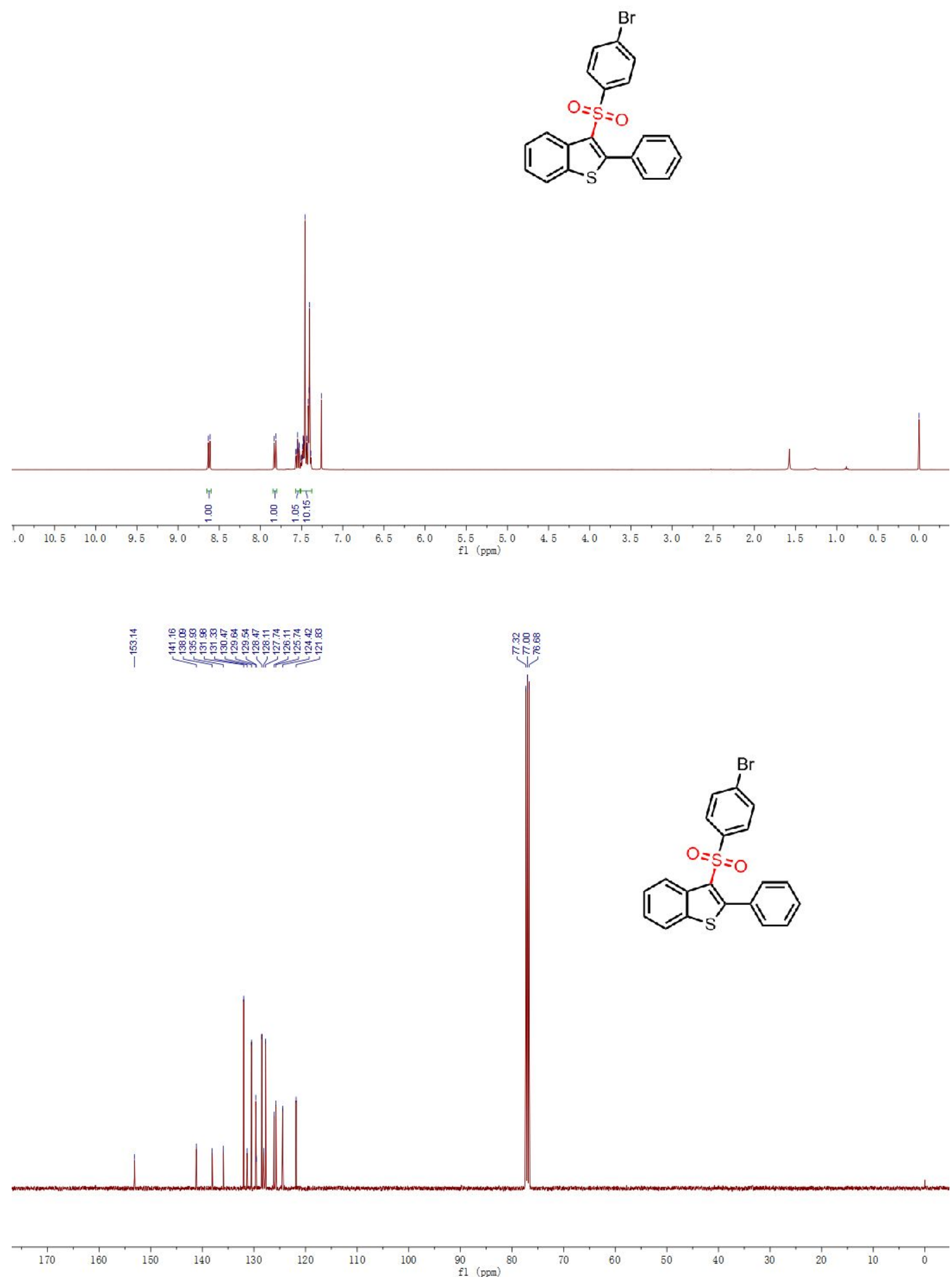

Figure S32. ${ }^{1} \mathrm{H}$ NMR of 3ai $\left(400 \mathrm{MHz}, \mathrm{CDCl}_{3}\right)$ and ${ }^{13} \mathrm{C}\left\{{ }^{1} \mathrm{H}\right\} \mathrm{NMR}$ of 3 ai $\left(100 \mathrm{MHz}, \mathrm{CDCl}_{3}\right)$ 
3-((4-Iodophenyl) sulfonyl)-2-phenylbenzo[b]thiophene (3aj)

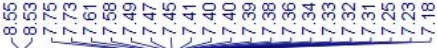
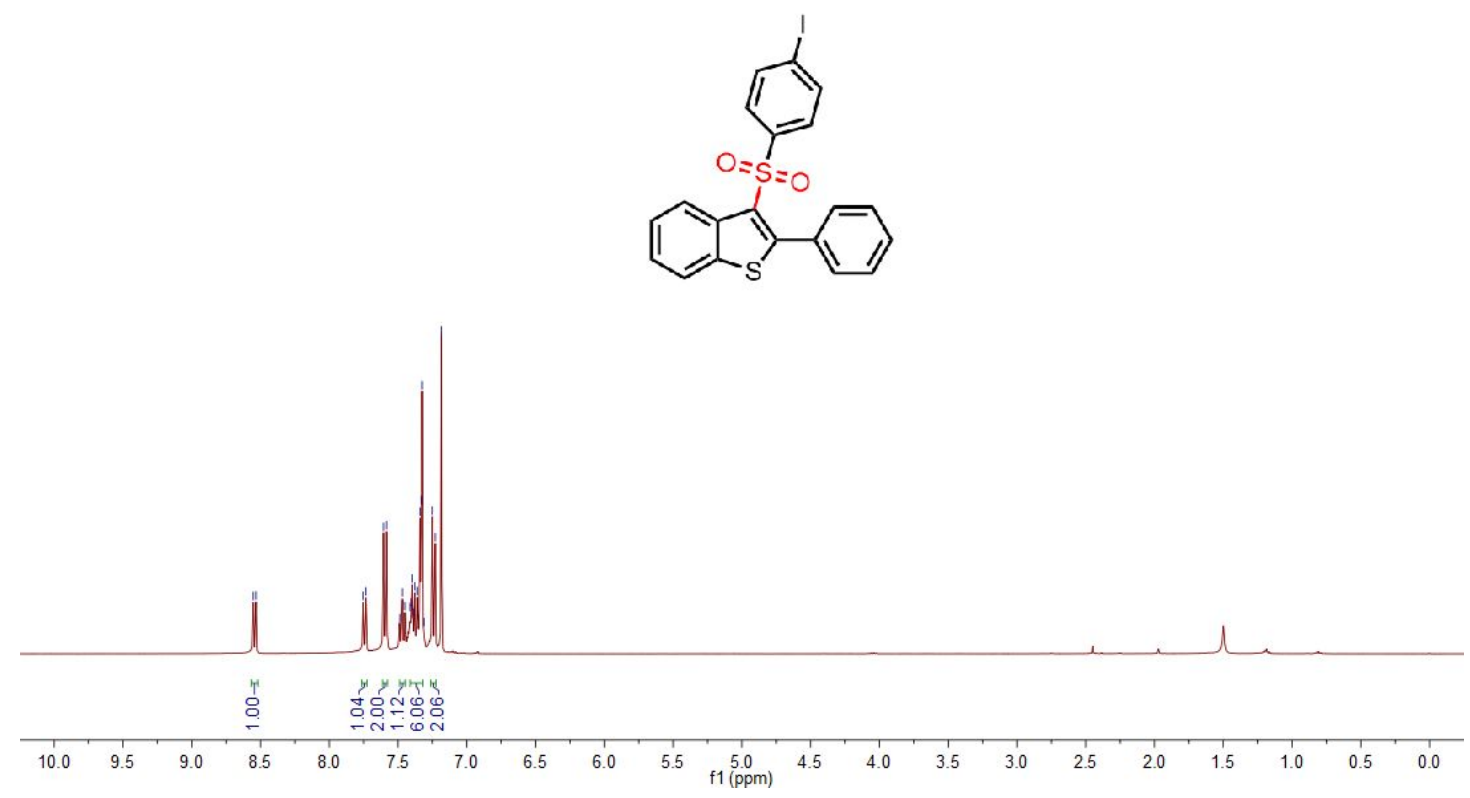

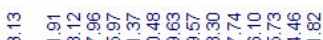

药
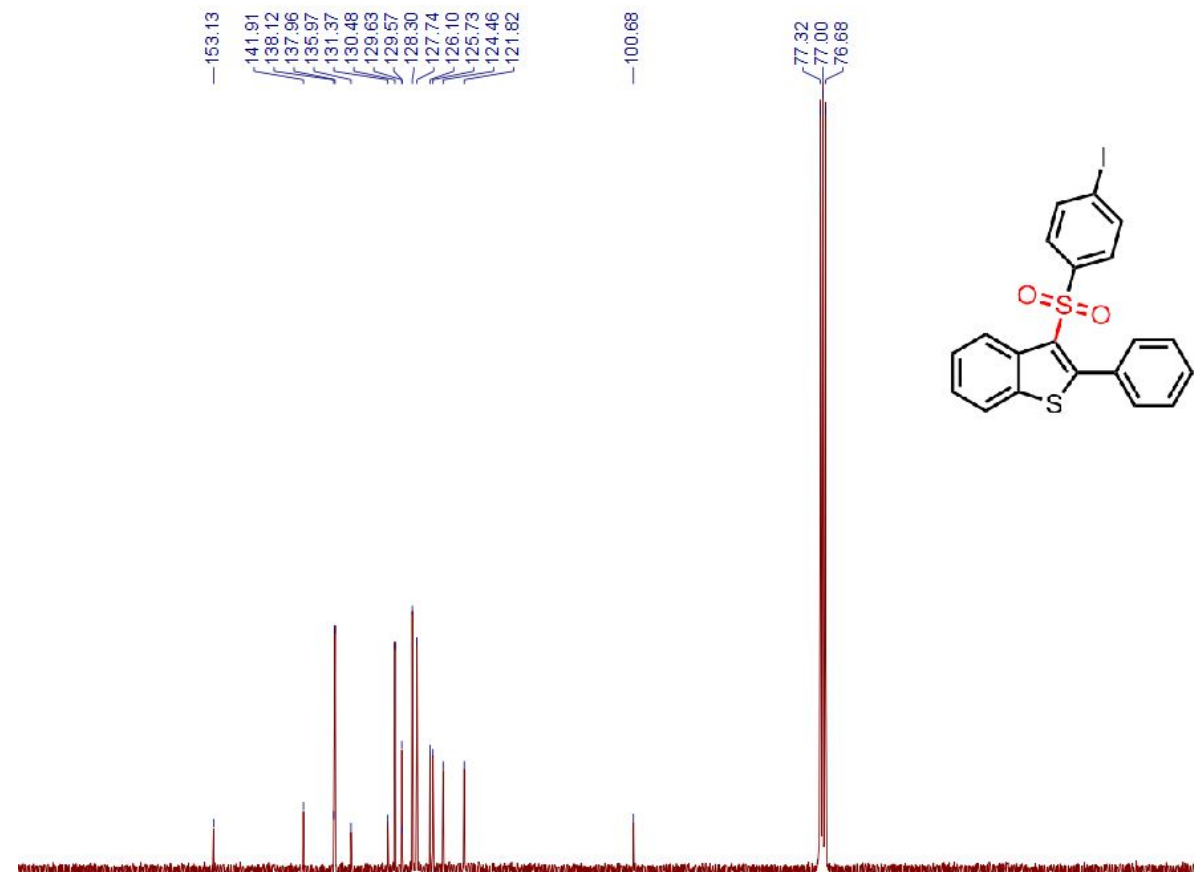

$\begin{array}{lllllllll}170 & 160 & 150 & 140 & 130 & 120 & 110 & 100 & 90 \\ \mathrm{f} 1(\mathrm{ppm}) & 80\end{array}$

Figure S33. ${ }^{1} \mathrm{H}$ NMR of 3 aj $\left(400 \mathrm{MHz}, \mathrm{CDCl}_{3}\right)$ and ${ }^{13} \mathrm{C}\left\{{ }^{1} \mathrm{H}\right\} \mathrm{NMR}$ of $\mathbf{3 a j}\left(100 \mathrm{MHz}, \mathrm{CDCl}_{3}\right)$ 
2-Phenyl-3-((4-(trifluoromethyl) phenyl) sulfonyl) benzo[b]thiophene (3ak)
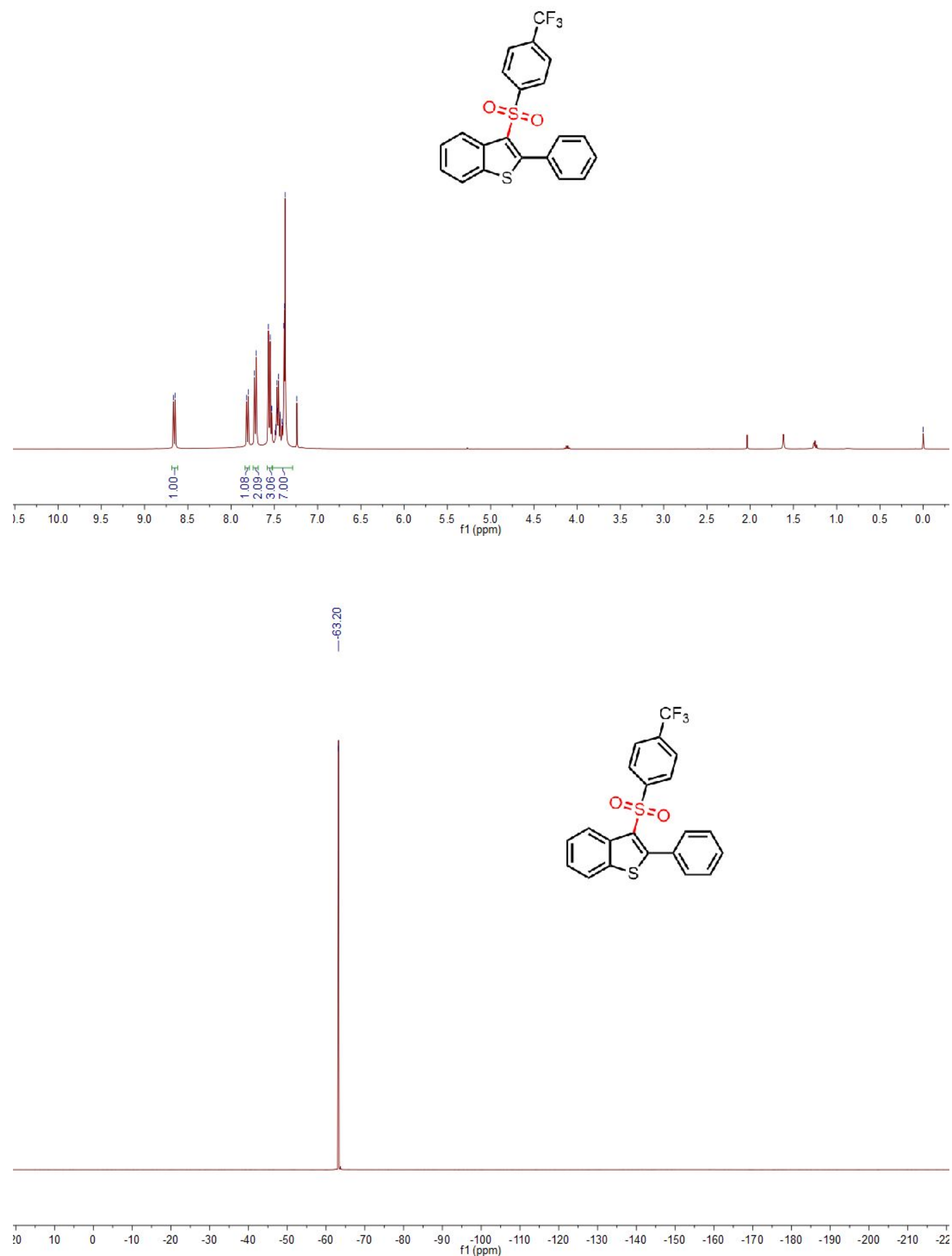

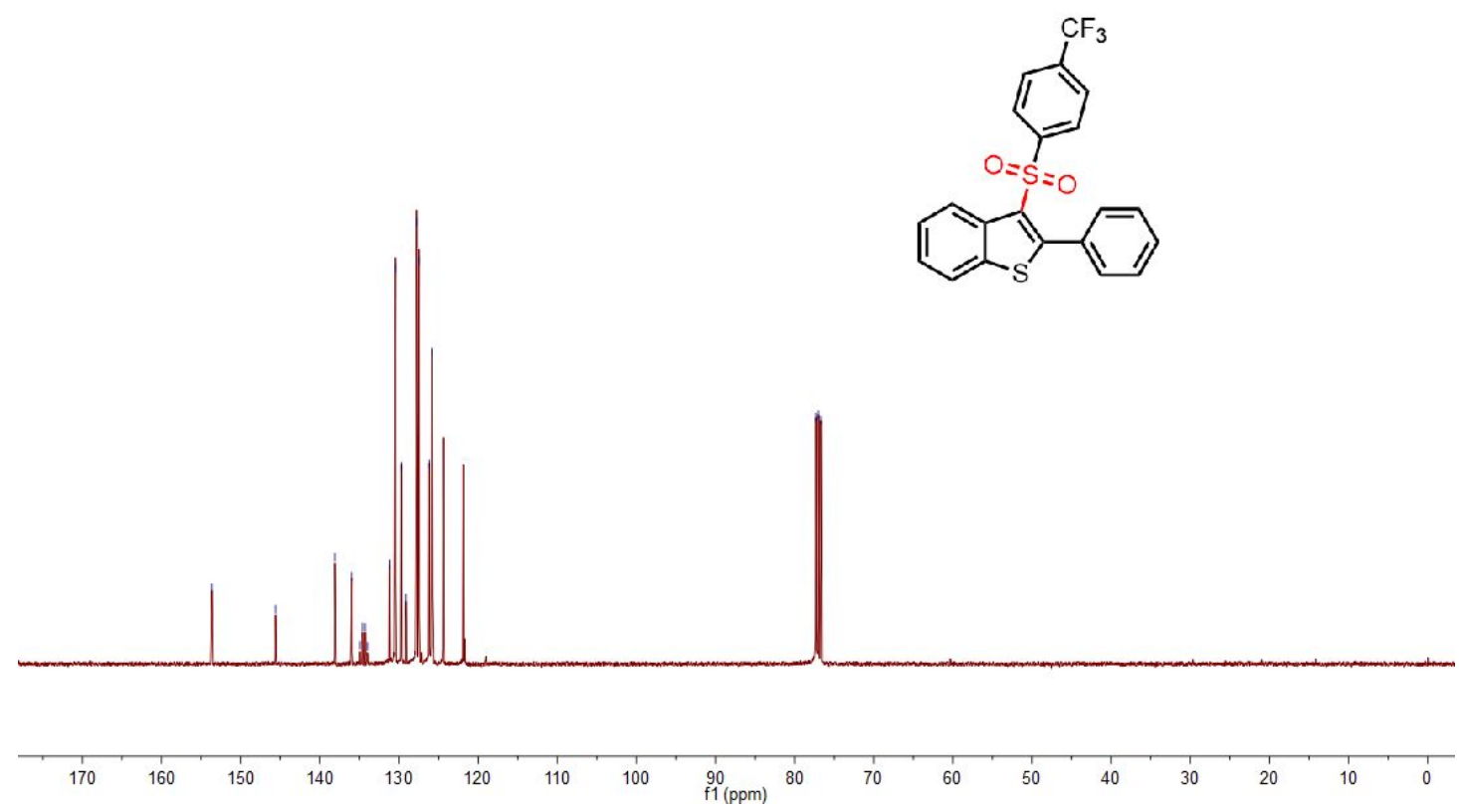

Figure S34. ${ }^{1} \mathrm{H}$ NMR of 3ak (400 MHz, $\left.\mathrm{CDCl}_{3}\right),{ }^{19} \mathrm{~F}$ NMR of 3ak $\left(376 \mathrm{MHz}, \mathrm{CDCl}_{3}\right.$ ) and ${ }^{13} \mathrm{C}\left\{{ }^{1} \mathrm{H}\right\}$ NMR of $\mathbf{3 a k}\left(100 \mathrm{MHz}, \mathrm{CDCl}_{3}\right)$

4-((2-Phenylbenzo[b]thiophen-3-yl) sulfonyl) benzonitrile (3al)

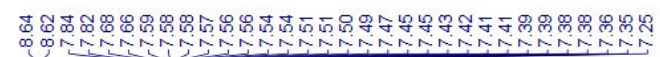
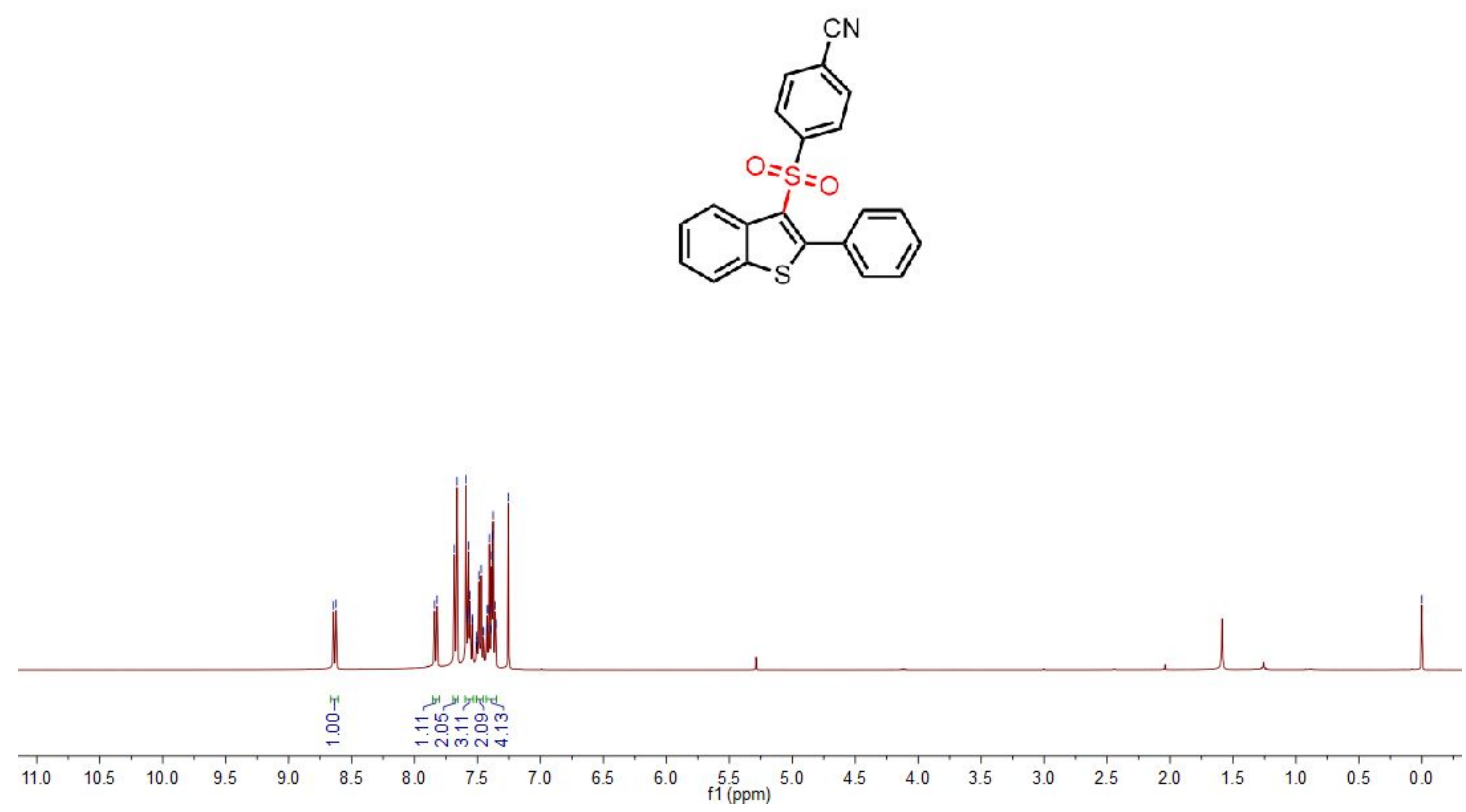

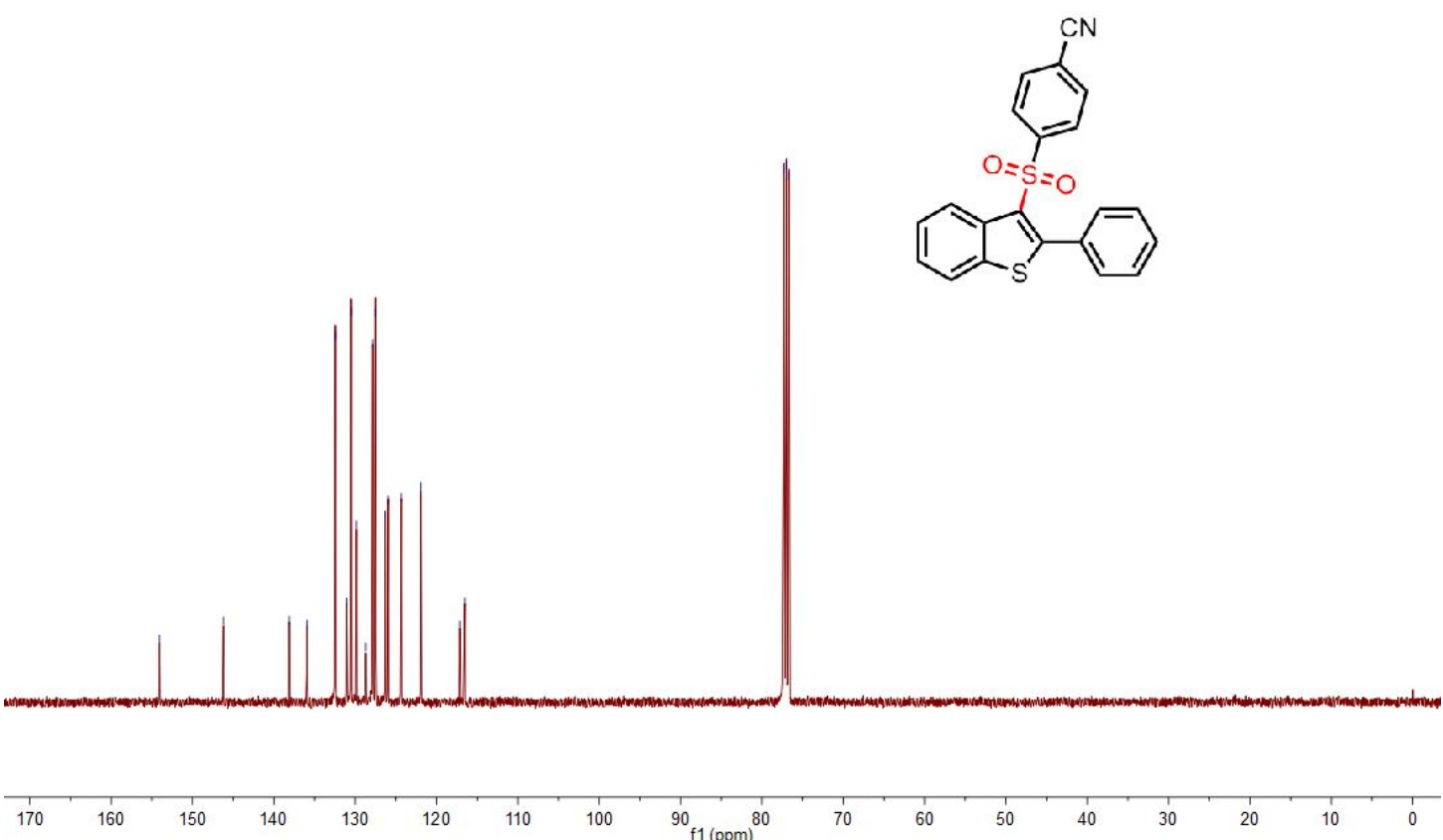

Figure S35. ${ }^{1} \mathrm{H}$ NMR of 3al $\left(400 \mathrm{MHz}, \mathrm{CDCl}_{3}\right)$ and ${ }^{13} \mathrm{C}\left\{{ }^{1} \mathrm{H}\right\}$ NMR of 3al $\left(100 \mathrm{MHz}, \mathrm{CDCl}_{3}\right)$

3-([1,1'-Biphenyl]-4-ylsulfonyl)-2-phenylbenzo[b]thiophene (3am)
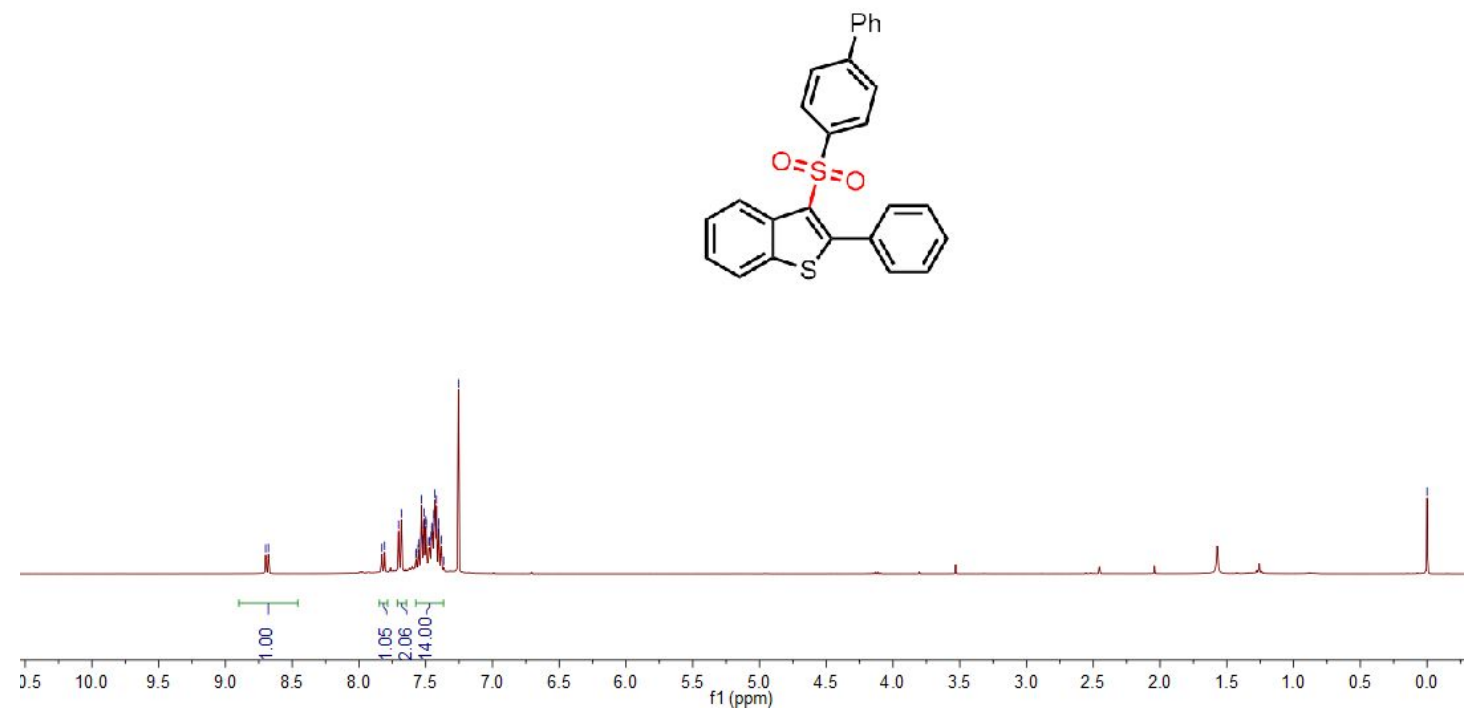


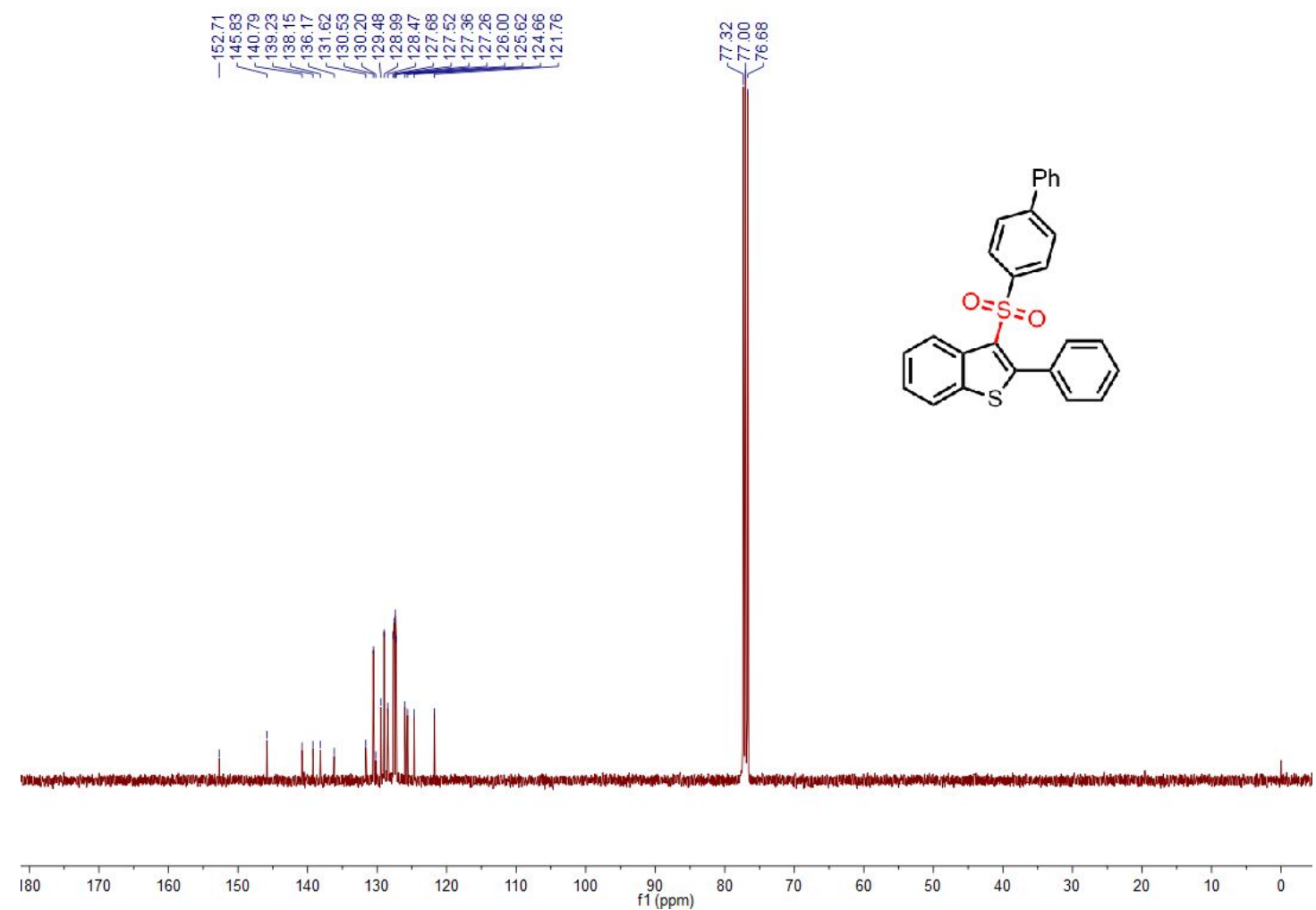

Figure S36. ${ }^{1} \mathrm{H}$ NMR of $3 \mathbf{a m}\left(400 \mathrm{MHz}, \mathrm{CDCl}_{3}\right)$ and ${ }^{13} \mathrm{C}\left\{{ }^{1} \mathrm{H}\right\} \mathrm{NMR}$ of $\mathbf{3 a m}\left(100 \mathrm{MHz}, \mathrm{CDCl}_{3}\right)$

3-(Naphthalen-2-ylsulfonyl)-2-phenylbenzo[b]thiophene (3an)

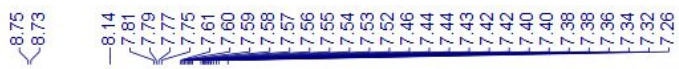
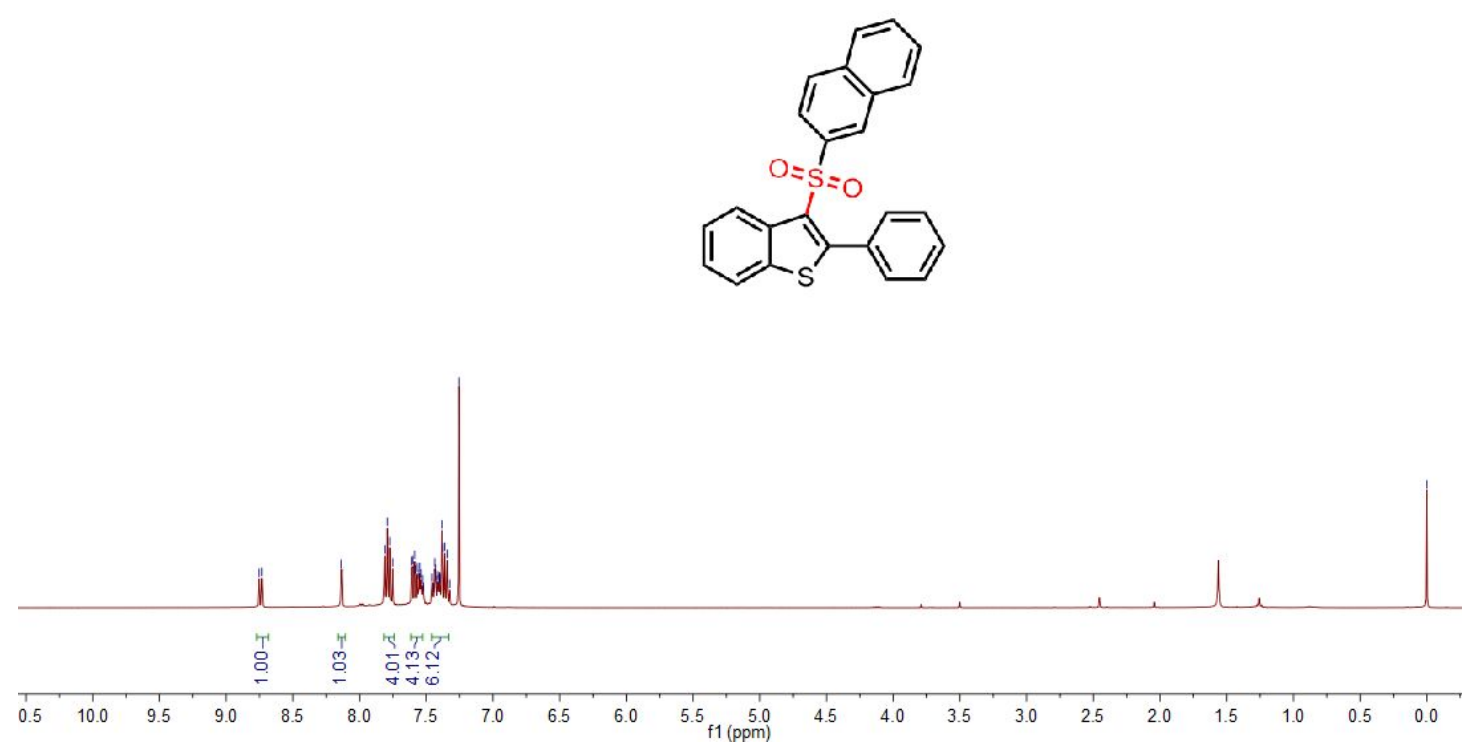

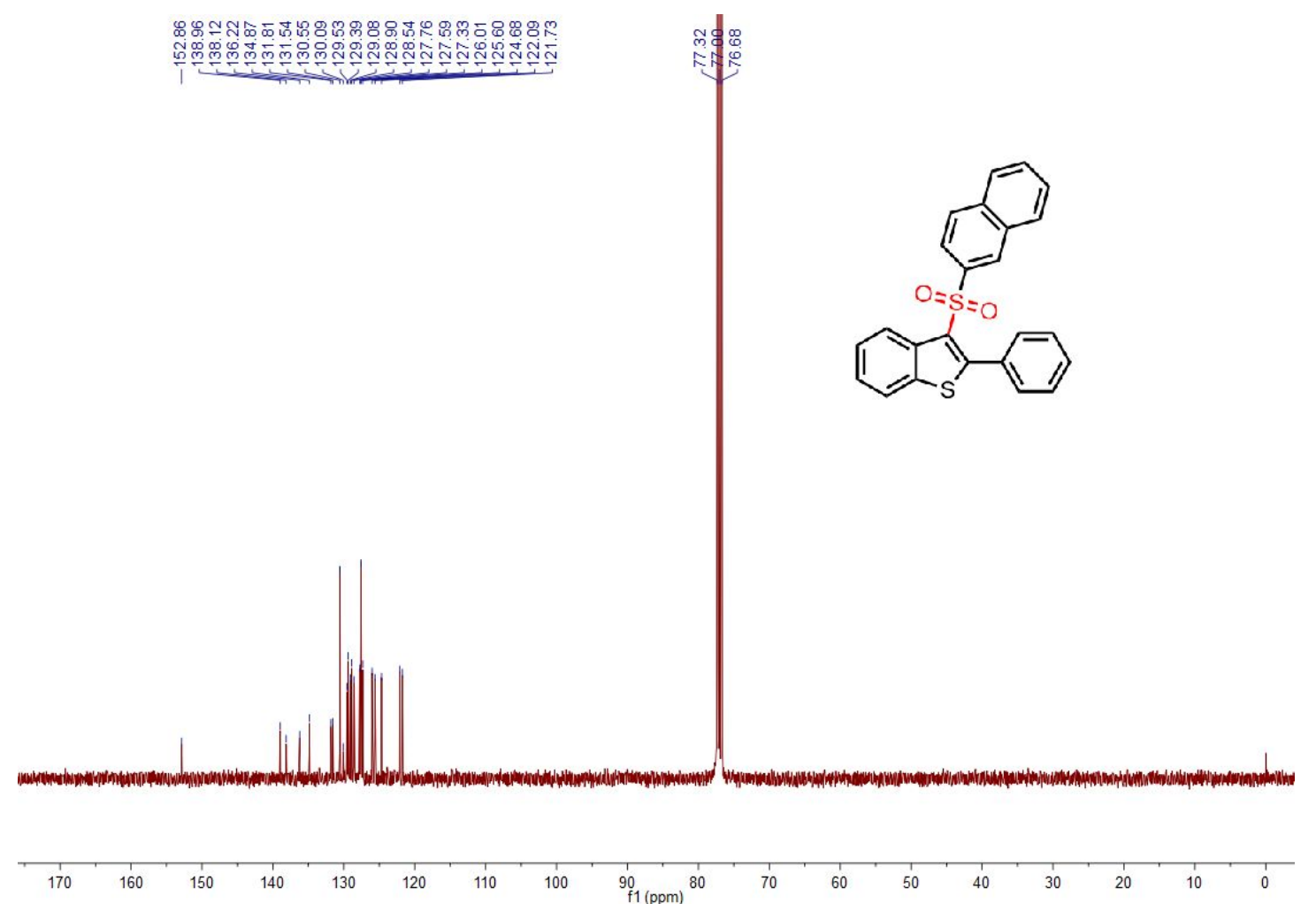

Figure S37. ${ }^{1} \mathrm{H}$ NMR of $\mathbf{3 a n}\left(400 \mathrm{MHz}, \mathrm{CDCl}_{3}\right)$ and ${ }^{13} \mathrm{C}\left\{{ }^{1} \mathrm{H}\right\}$ NMR of $\mathbf{3 a n}\left(100 \mathrm{MHz}, \mathrm{CDCl}_{3}\right)$

2-Phenyl-3-(thiophen-2-ylsulfonyl) benzo[b]thiophene (3ao)

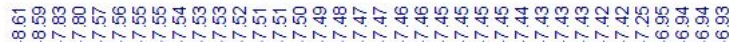
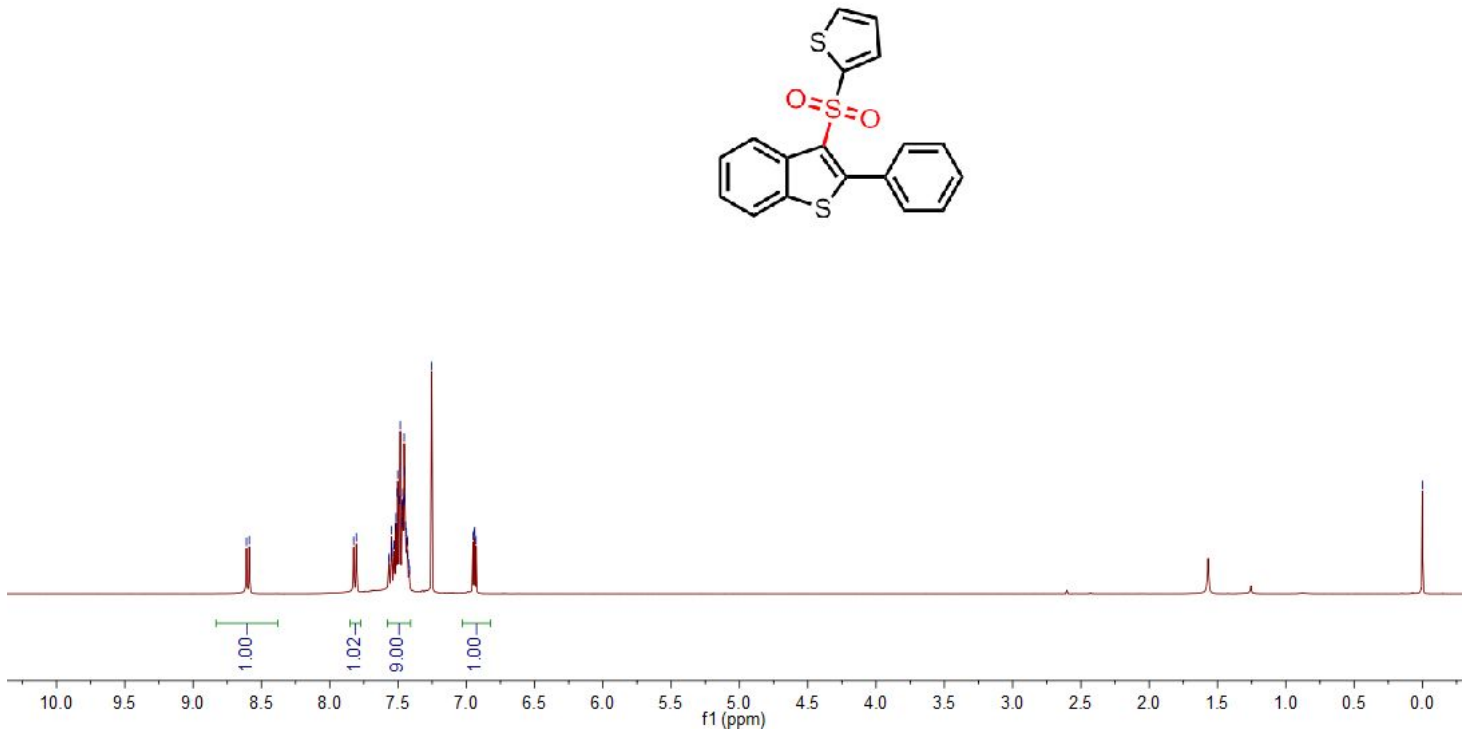


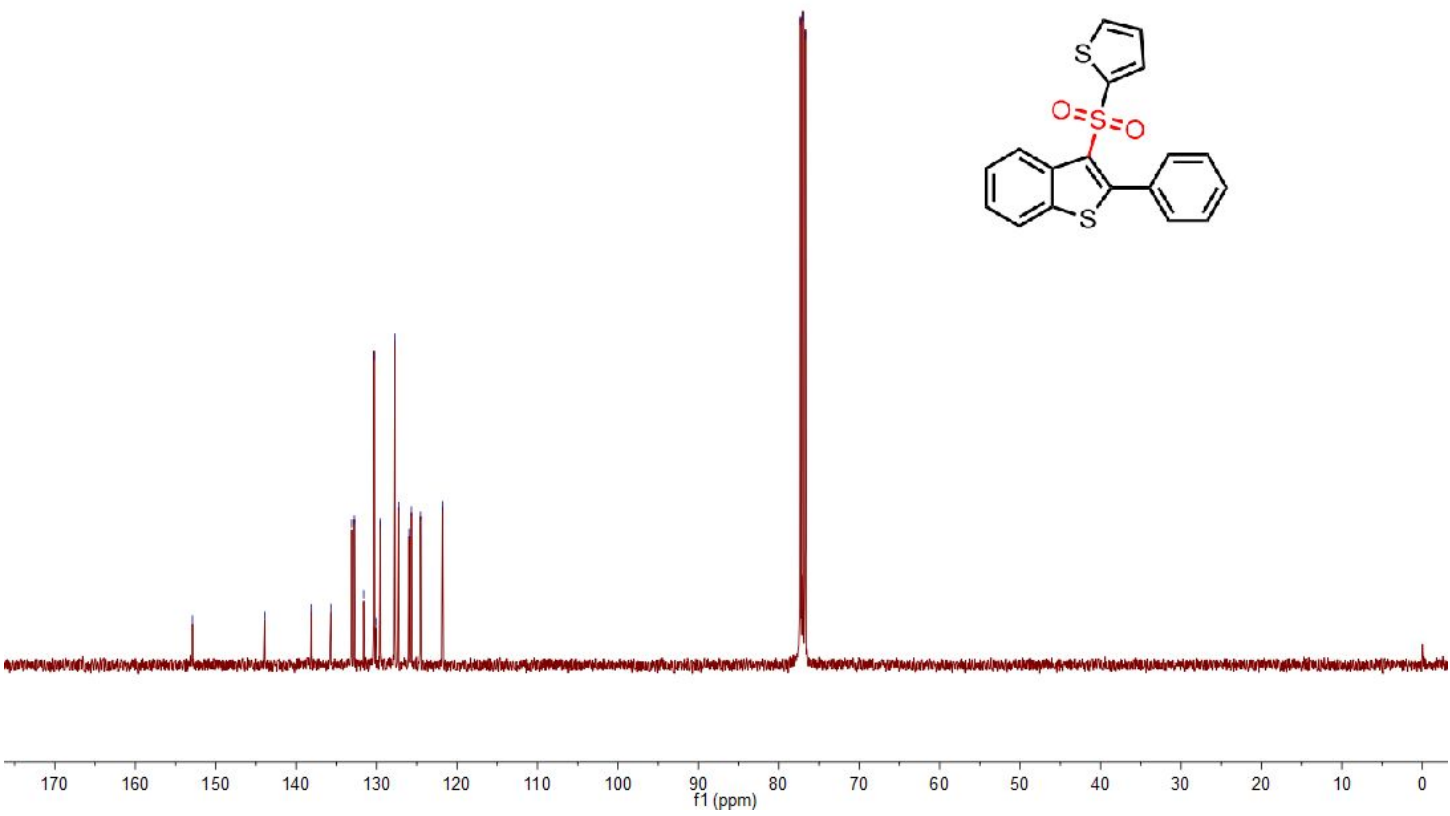

Figure S38. ${ }^{1} \mathrm{H}$ NMR of $3 \mathbf{a o}\left(400 \mathrm{MHz}, \mathrm{CDCl}_{3}\right)$ and ${ }^{13} \mathrm{C}\left\{{ }^{1} \mathrm{H}\right\} \mathrm{NMR}$ of $\mathbf{3 a o}\left(100 \mathrm{MHz}, \mathrm{CDCl}_{3}\right)$

3-(Cyclopropylsulfonyl)-2-phenylbenzo[b]thiophene (3ap)

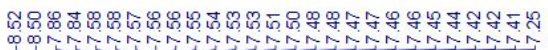

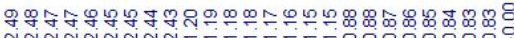
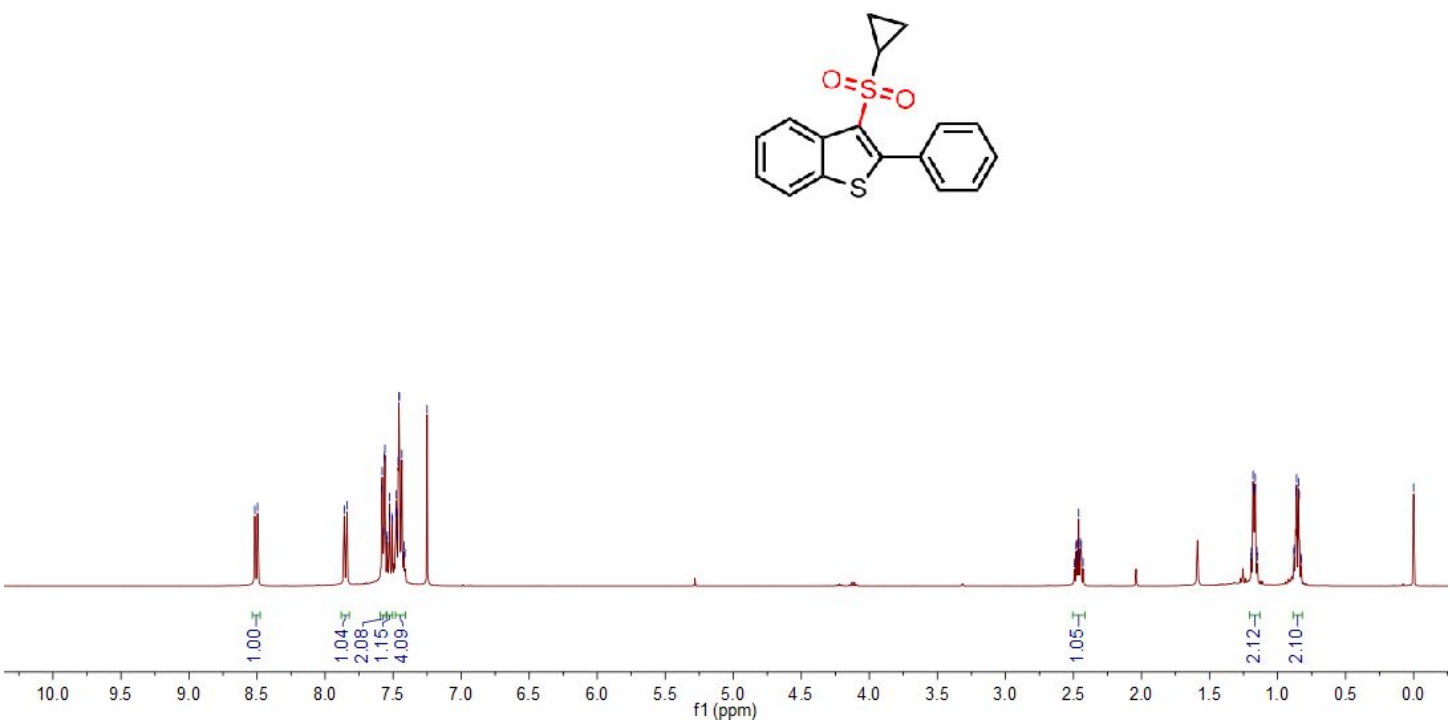

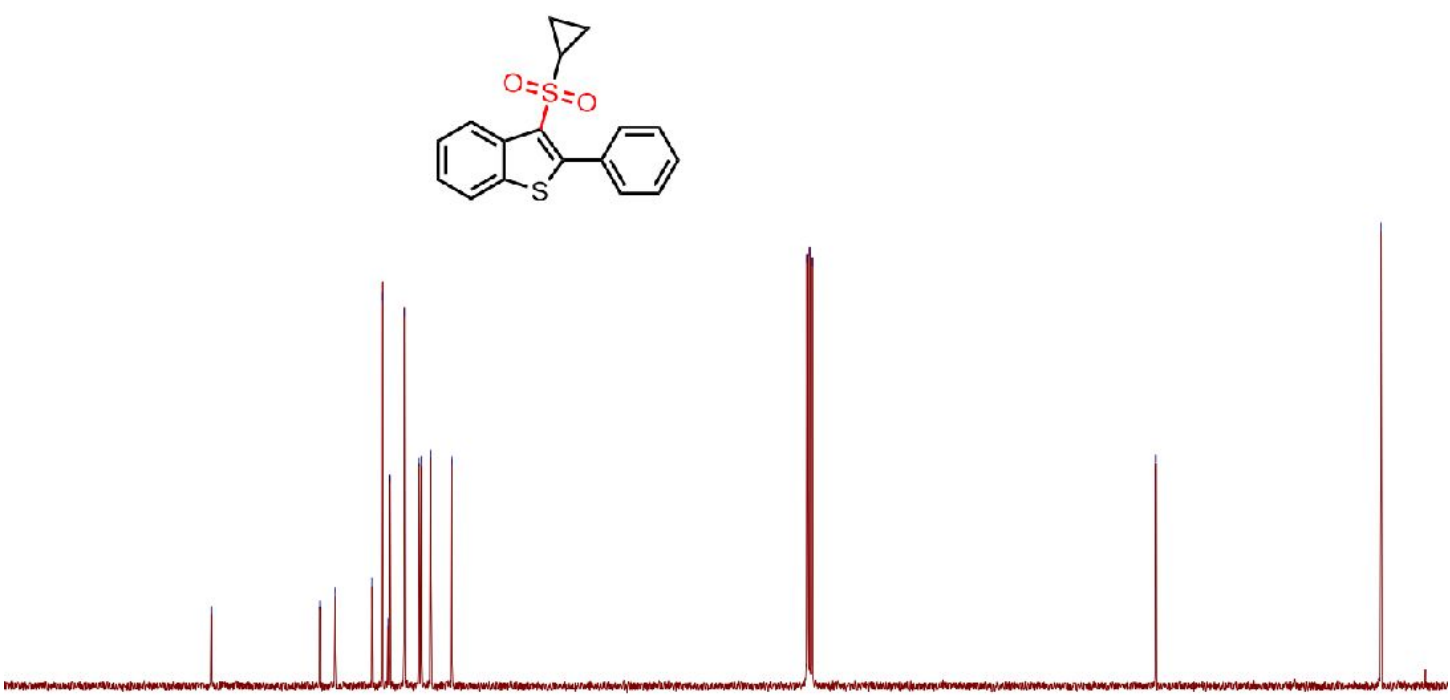

$170 \quad 160$

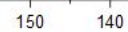

$\left.\mathrm{f1}^{90} \mathrm{ppm}\right)$

Figure S39. ${ }^{1} \mathrm{H}$ NMR of 3 ap $\left(400 \mathrm{MHz}, \mathrm{CDCl}_{3}\right)$ and ${ }^{13} \mathrm{C}\left\{{ }^{1} \mathrm{H}\right\} \mathrm{NMR}$ of $\mathbf{3 a p}\left(100 \mathrm{MHz}, \mathrm{CDCl}_{3}\right)$

3-(Methylsulfonyl)-2-phenylbenzo[b]thiophene (3aq)
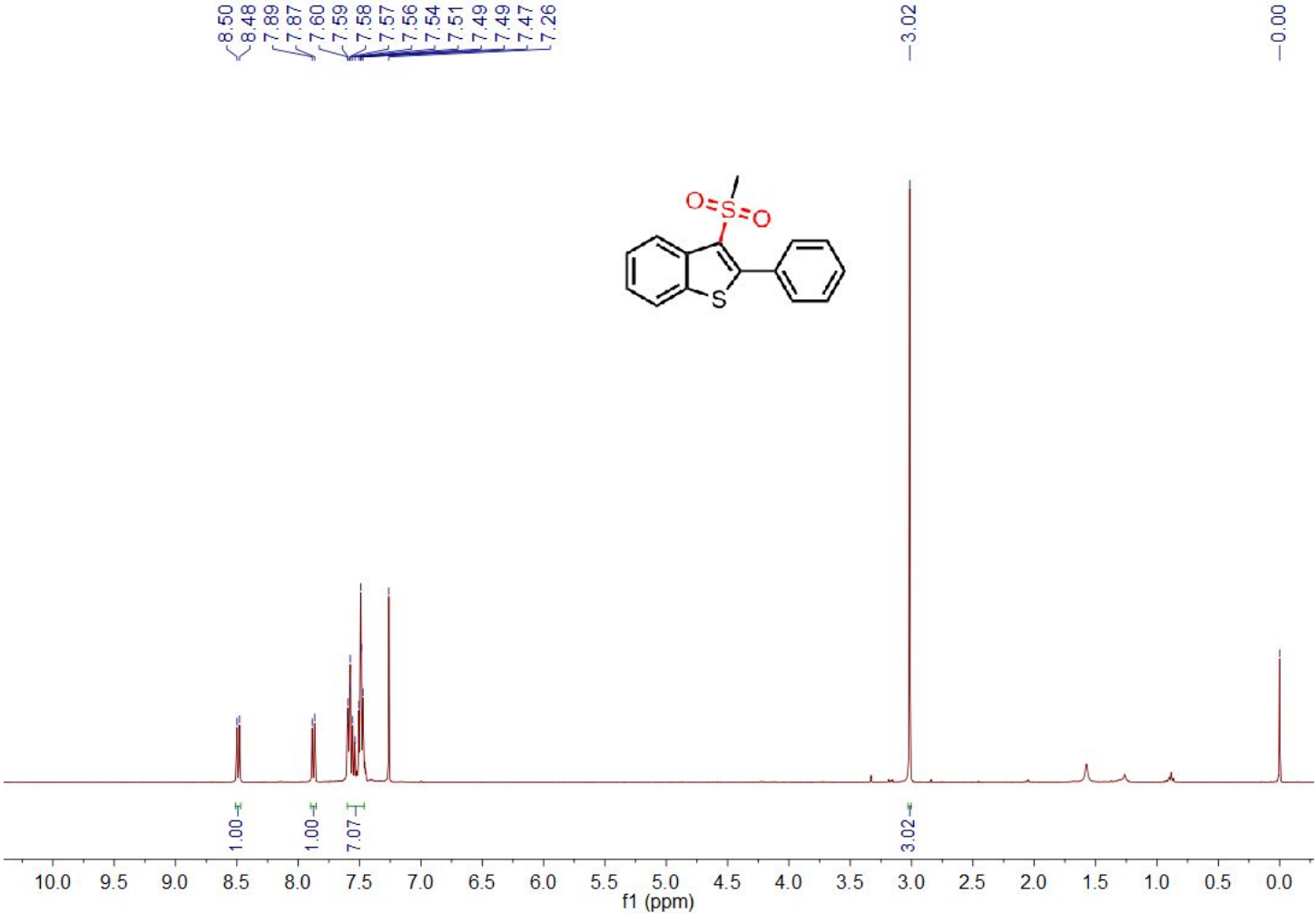


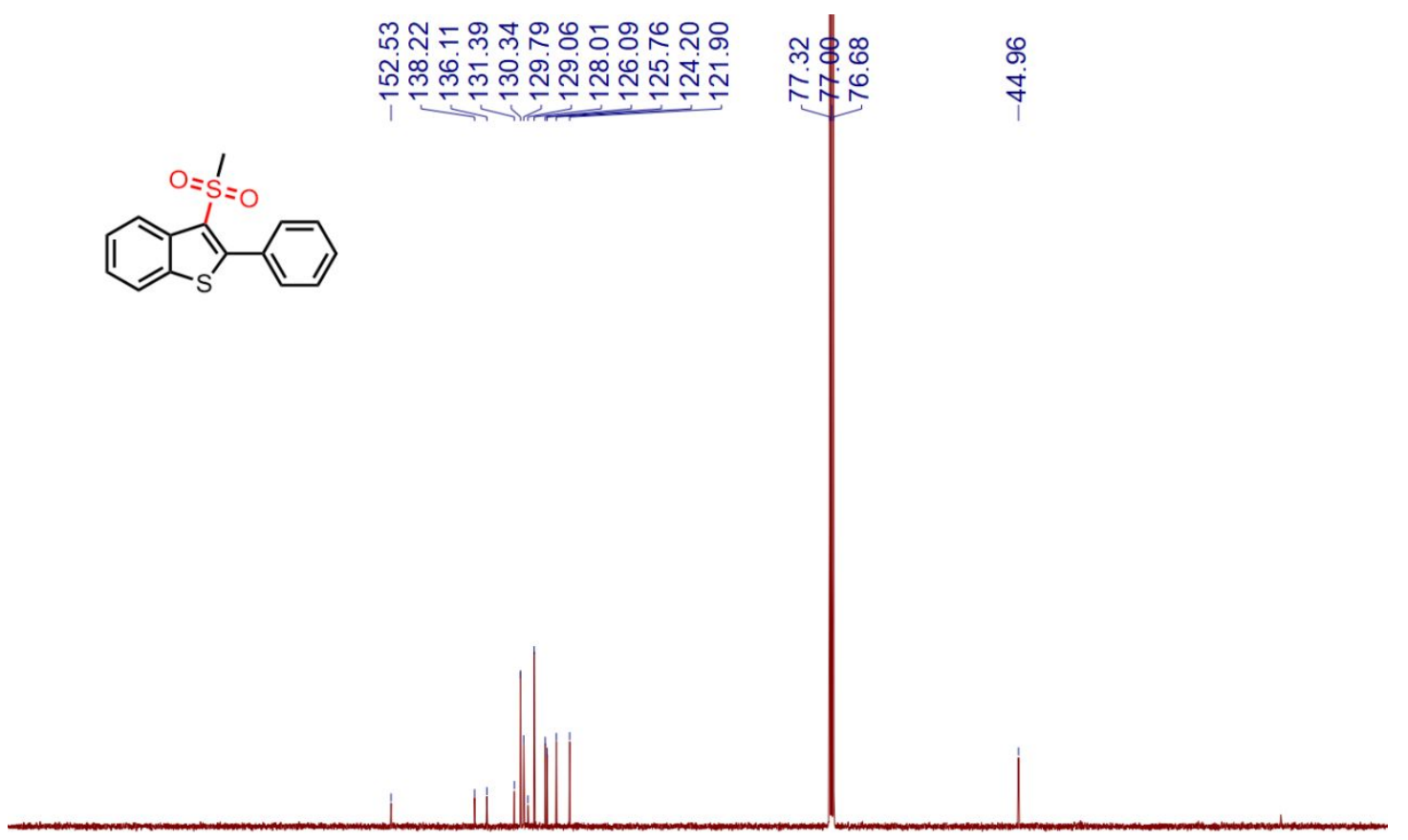

$\begin{array}{llllllllllllllllllllll}210 & 200 & 190 & 180 & 170 & 160 & 150 & 140 & 130 & 120 & \begin{array}{c}110 \\ \mathrm{f} 1\end{array}\left(\begin{array}{l}100 \\ (\mathrm{ppm})\end{array}\right. & 90 & 80 & 70 & 60 & 50 & 40 & 30 & 20 & 10 & 0 & -10\end{array}$

Figure S40. ${ }^{1} \mathrm{H}$ NMR of $\mathbf{3 a q}\left(400 \mathrm{MHz}, \mathrm{CDCl}_{3}\right)$ and ${ }^{13} \mathrm{C}\left\{{ }^{1} \mathrm{H}\right\} \mathrm{NMR}$ of $\mathbf{3 a q}\left(100 \mathrm{MHz}, \mathrm{CDCl}_{3}\right)$

3-(Ethylsulfonyl)-2-phenylbenzo[b]thiophene (3ar)

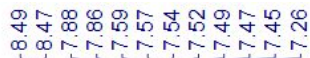
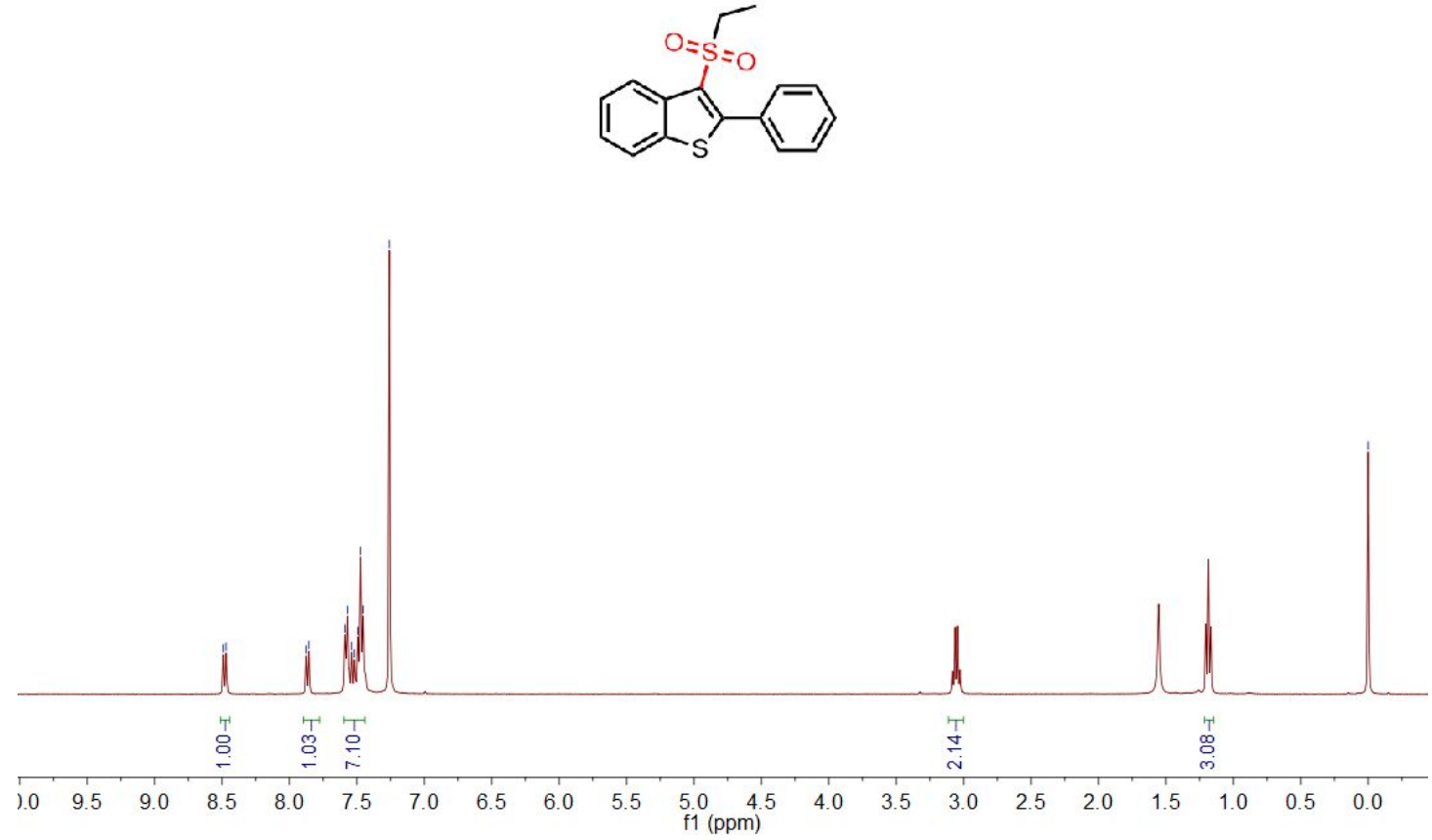


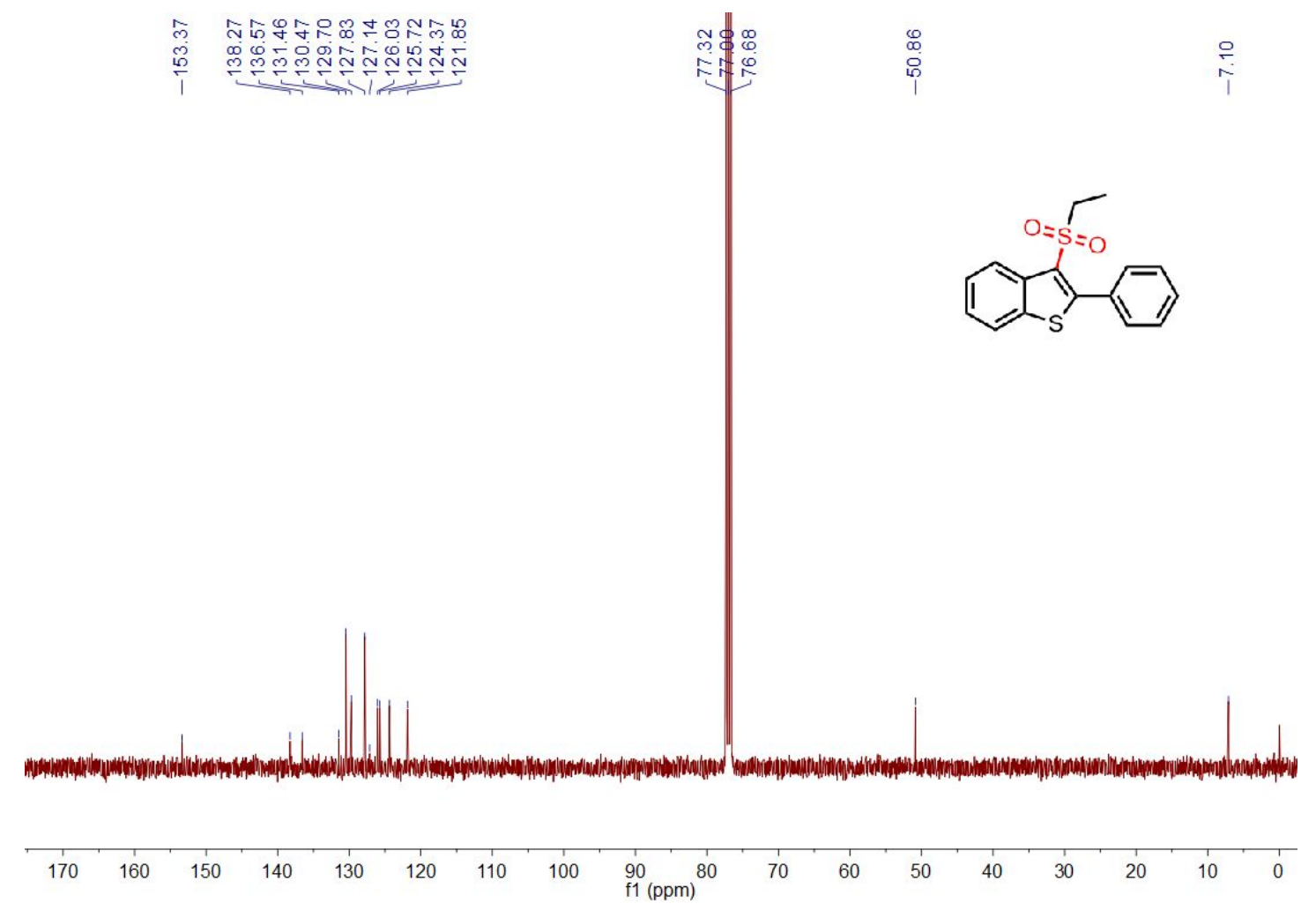

Figure S41 ${ }^{1} \mathrm{H}$ NMR of $\mathbf{3 a r}\left(400 \mathrm{MHz}, \mathrm{CDCl}_{3}\right)$ and ${ }^{13} \mathrm{C}\left\{{ }^{1} \mathrm{H}\right\} \mathrm{NMR}$ of $\mathbf{3 a r}\left(100 \mathrm{MHz}, \mathrm{CDCl}_{3}\right)$

(2-Tosylethene-1,1-diyl) dibenzene (4)

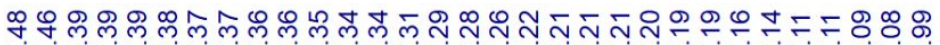

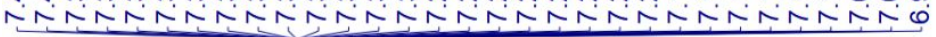<smiles>Cc1ccc(S(=O)(=O)C=C(c2ccccc2)c2ccccc2)cc1</smiles>

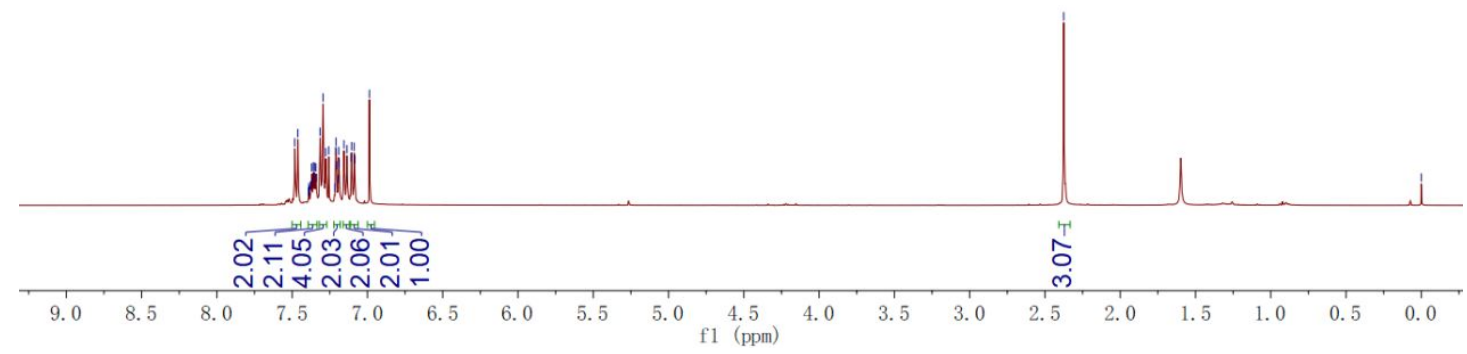


<smiles>Cc1ccc(S(=O)(=O)C=C(c2ccccc2)c2ccccc2)cc1</smiles>

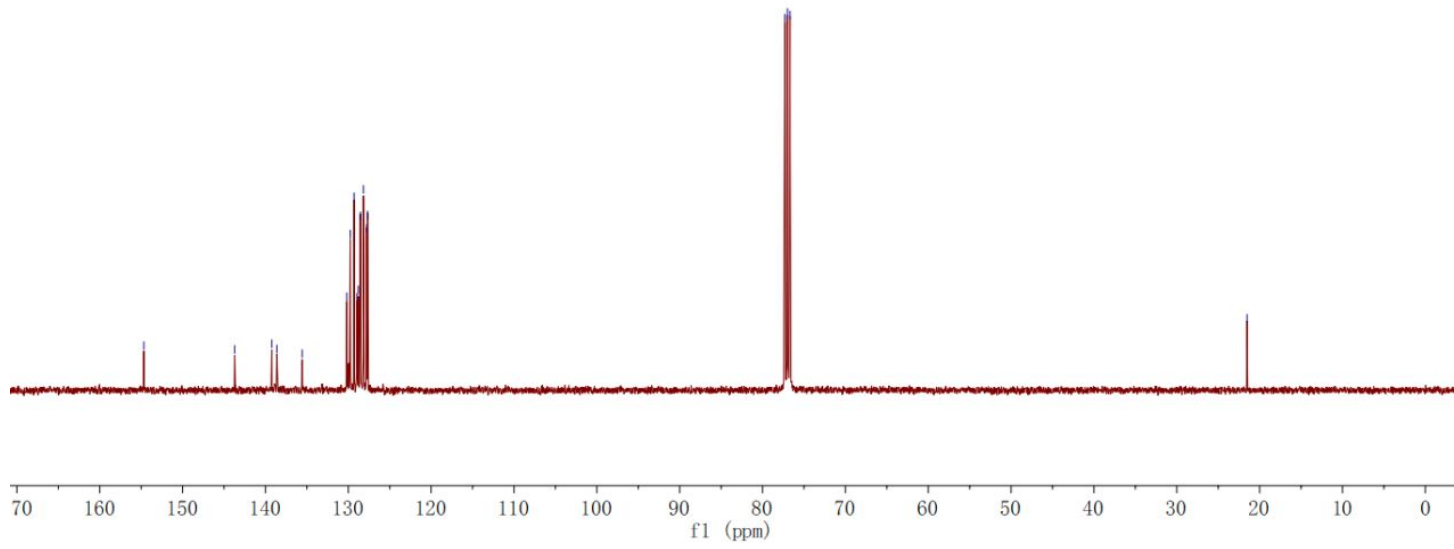

Figure S42 ${ }^{1} \mathrm{H}$ NMR of $4\left(400 \mathrm{MHz}, \mathrm{CDCl}_{3}\right)$ and ${ }^{13} \mathrm{C}\left\{{ }^{1} \mathrm{H}\right\} \mathrm{NMR}$ of $4\left(100 \mathrm{MHz}, \mathrm{CDCl}_{3}\right)$ 